\title{
The Variability of Perfusion Index as a New Parameter in Different Types of Anaesthesia Techniques and Its Correlation with Surgical Stress and Recovery From Anesthesia: An Observational Clinical Study
}

\author{
Authors \\ Dr Amit Kumar MD (Anesthesiology), Dr A.V. Nadkarni MD,D.A. (Anesthesiology) \\ KIMS, KARAD \\ Corresponding Author \\ Mr Amit Kumar \\ Email: amit.kmc1979@gmail.com,Mobile: 9130226814 \\ Room no 112 Krishna Institute of Medical Sciences Karad, Maharashtra 415539 India
}

\section{INTRODUCTION}

Perfusion index is an indirect, non invasive, numerical, dynamic and continuous measure of peripheral perfusion obtained from pulse oximeter that provides useful information to the physician in several clinical settings. Perfusion index is reliable and easier alternative for detection of stress response during induction ${ }^{59}$ and peri-operative period of operation and has a prognostic value in predicting peri-operative outcome. ${ }^{65}$

Trends in change of perfusion index reveals even the subtle changes in peripheral circulation that is otherwise missed in static display.

In anaesthetic settings spike in perfusion index indicates onset of anaesthesia at the physiological level and gives physicians one more tool to further consolidate onset of anaesthesia ${ }^{15,19,44,48,64}$.Peripheral Perfusion index alterations are more marked in patients who are likely to develope severe complications. Studies are also showing that treatment based on perfusion index can lead to improved patient outcome.

What is perfusion index?

Perfusion index is ratio of pulsatile blood flow to non pulsatile blood flow in person`s peripheral tissue such as finger tip, ear-lobes.

Calculation of the perfusion index

The pulse oximetry plethysmographic (POP) waveform relies on red and infrared light absorption which include two components. The first component is said to be constant amount of light (direct current: DC), which is absorbed by skin, bone, tissue, pigment and non- pulsatile blood. The second component is said to be a variable amount of light (alternating current: AC). It is measured by pulsatile arterial blood flow .For P.I. calculation ,the infrared pulsatile signal is indexed against non-pulsatile infrared signal and expressed as a percentage (P.I. $=\{$ AC/DC $\} 100)$ reflecting the amplitude of the POP waveform.

$$
\mathrm{PI}=\frac{\mathrm{AC}_{\mathrm{IR}}}{\mathrm{DC}_{\mathrm{IR}}} \times 100 \% \text {. }
$$


Efficacy of P.I. is more in detecting haemodynamic stress response to insertion of i-gel, laryngeal mask airway and endotracheal tube and more reliable than other parameter to detect surgical stress response during general anaesthesia. Its efficacy is $100 \% .^{18,12,7,8,59}$

It is valuable noninvasive objective during anaesthetic practice to find out methods for predicting haemodynamic response to anaesthetic drugs techniques and to intra-operative stimuli.A practically applicable method of evaluating sympathetic tone and responsiveness is of clinical utility. ${ }^{2,4}$

Although confounders influencing peripheral perfusion are hypothermia, vasoconstriction and sympathetic

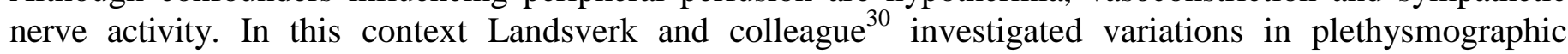
waveform amplitudes in deeply sedated patient and mechanically ventilated patients. They observed slow and large spontaneous microcirculation per laser Doppler flow metry related to sympathetic nervous system and presumed this mechanism determined the large variability of pulse oximeter photo-plethysmograph waveform signal.

Our overall aim of the research is to establish perfusion index as a new parameter to monitor haemodynamic changes in different techniques of anaesthesia and its relation with onset of anaesthesia. Clinical studies in adults and paediatric patients have demonstrated that an increase in perfusion index is an early indicator that anaesthesia has initiated peripheral vasodilation that typically occurs before onset of anaesthetic effects. Thus detection of the spikes of perfusion index will indicate that anaesthesia has started working .If trends and patterns of changes in perfusion index is ably intercepted, can give immediate feedback to the changes in peripheral perfusion.

Most anaesthetic agents work by causing peripheral vasodilation and also there is redistribution of temperature. Measuring perfusion index gives real time changes in perfusion of the patients and is also a indicator of whether anaesthesia is working or not. ${ }^{15,19}$

\section{IMPORTANCE OF OBJECTIVES}

Estabilishing the predictive value of the perfusion index regarding incidence of hypotension in neuraxial anaesthesia will alert anaesthetist to be more vigilant and well prepared.

Increasing trends in perfusion index will predict the onset of anaesthesia in neuraxial block and it will further consolidate the onset of anaesthesia which is done by conventional methods thus helping physicians to concentrate on other aspects of operation with greater ease. Also a flat P.I. curve in neuraxial anaesthesia will warn about failure of anaesthesia. ${ }^{19}$

It will give continuous and real time feed- back about the peripheral circulatory status of the patients thus helping physicians to make informed decisions about the patient management particularly fluid management of the patients. ${ }^{22}$

Study of relation between perfusion index and urine output during anaesthesia can throw light on BODY FLUID DYNAMICS during anaesthesia and overall homeostasis. ${ }^{21,22,30}$

Maintenance of P.I. to optimum level throughout surgical procedure will result into smoother and quicker recovery of patients, with minimal postoperative complications and better surgical outcome.

Potential future applications for monitoring perfusion index

Perfusion index will give indication of circulatory functions of re-implanted body parts such as fingers or hands. It will also inform about restoration of peripheral perfusion after cardiopulmonary bypass. Other potential uses may be estimation of volume depletion in trauma patients.

\section{REASONS FOR SELECTION OF THIS TOPIC}

1) Conventional parameter like blood pressure, pulse rate and $\mathrm{SPO}_{2}$ are less of a direct measurement of the peripheral circulatory status and perfusion of the patients. Continuous and real time feed -back of circulatory status of patients will help making more informed decision regarding patient management.

2) Establishing correlation between baseline perfusion index and hypotension after spinal anaesthesia will help anticipating the dramatic fall of BP and its management.

3) Today, great emphasis is given to a recently recognised condition in patients under-going surgery and anaesthesia which is known as SURGICAL STRESS RESPONSES .It is characterised by profound sympathetic stimulation with high level of catecholamines, cortisol and other stress 
hormone secretions and is responsible for intra and postoperative catabolic states, with all its deleterious effects on various regional tissue circulations and on cellular homeostasis of whole body. The magnitude of this stress response decides the nature of patient's recovery from anaesthetic state, the post operative complications, patient`s well being, recovery of patients, hospital stay and the cost, incidence of morbidity and even mortality .Since P.I. indicates peripheral vascular resistance which is directly related to sympathetic tone and catecholamine levels, it can be a good parameter to assess magnitude of surgical stress responses and anaesthetists can effectively counteract the same or obtund it to ensure optimum P.I. which should result in better surgical outcome on many counts .Few anaesthetic techniques like spinal and epidural anaesthesia have been shown to be the choice of techniques for some specific surgical procedures so far as to avoid surgical stress responses resulting in better surgical outcomes. Hence, it is important to explore and compare P.I.in different anaesthetic techniques and correlate their outcomes and assess role of P.I.as a diagnostic and guiding tool in management of anaesthesia in patients subjected to surgeries. ${ }^{35,39}$

\section{AIMS AND OBJECTIVES}

- To monitor perfusion index perioperatively in each patient.

- Correlation of perfusion index with other haemodynamic prameters observed simultaneously.

- To find out variation in perfusion index with different types of anesthesia - neuraxial, inhalational, TIVA and ETGA.

- To conclude which anaesthetic technique offer minimal P.I. changes and prove safeguard against surgical stress response.

\section{REVIEW OF LITERATURE HISTORY AND EVOLUTION OF PERFUSION INDEX}

In 1935, Karl Matthes (German physician 1905-1962) developed the first 2-wavelength ear O2 saturation meter with red and green filters (later switched to red and infrared filters). His meter was the first device to measure $\mathrm{O}_{2}$ saturation.

The original oximeter was made by Glenn Allan Millikan in the 1940s.In 1949 Wood added a pressure capsule to squeeze blood out of ear to obtain zero setting in an effort to obtain absolute $\mathrm{O} 2$ saturation value when blood was re-admitted. The concept was similar to today's conventional pulse oximetry but was difficult to implement because of unstable photocells and light sources. This method is not used clinically. In 1964 Shaw assembled the first absolute reading ear oximeter by using eight wavelengths of light. Commercialized by Hewlett-Packard, its use was limited to pulmonary functions and sleep laboratories due to cost and size.

Pulse oximetry was developed in 1972, by Takuo Aoyagi and Michio Kishi, bioengineers, at Nihon Kohden using the ratio of red to infrared light absorption of pulsating components at the measuring site. Susumu Nakajima, a surgeon, and his associates first tested the device in patients, reporting it in 1975. It was commercialized by Biox in 1981 and Nellcor in 1983. Biox was founded in 1979, and introduced the first pulse oximeter to commercial distribution in 1981. Biox initially focused on respiratory care, but when the company discovered that their pulse oximeters were being used in operating rooms to monitor oxygen levels, Biox expanded its marketing resources to focus on operating rooms in late 1982. A competitor, Nellcor (now part of Covidien, Ltd.), began to compete with Biox for the U.S. operating room market in 1983. Prior to the introduction of pulse oximetry, a patient's oxygenation could only be determined by arterial blood gas, a single-point measurement that takes several minutes for sample collection and processing by a laboratory. In the absence of oxygenation, damage to the brain starts within 5 minutes with brain death ensuing within another 10-15 minutes. The worldwide market for pulse oximetry is over a billion dollars. With the introduction of pulse oximetry, a non-invasive, continuous measure of patient's oxygenation was possible, revolutionizing the practice of anesthesia and greatly improving patient safety. Prior to its introduction, studies in anaesthesia journals estimated U.S. patient mortality as a consequence of undetected hypoxemia at 2,000 to 10,000 deaths per year, with no known estimate of patient morbidity.

By 1987, the standard of care for the administration of a general anaesthetic in the U.S. included pulse oximetry. From the operating room, the use of pulse oximetry rapidly spread throughout the hospital, first to 
the recovery room, and then into the various intensive care units. Pulse oximetry was of particular value in the neonatal unit where the patients do not thrive with inadequate oxygenation, but too much oxygen and fluctuations in oxygen concentration can lead to vision impairment or blindness from retinopathy of prematurity (ROP). Furthermore, obtaining an arterial blood gas from a neonatal patient is painful to the patient and a major cause of neonatal anemia.Motion artifact can be a significant limitation to pulse oximetry monitoring resulting in frequent false alarms and loss of data. The reason for this is that during motion and low peripheral perfusion, many pulse oximeters cannot distinguish between pulsating arterial blood and moving venous blood, leading to underestimation of oxygen saturation. Early studies of pulse oximetry performance during subject motion made clear the vulnerabilities of conventional pulse oximetry technologies to motion artifact. In 1995, Masimo introduced Signal Extraction Technology (SET) that could measure accurately during patient motion and low perfusion by separating the arterial signal from the venous and other signals. Since then, pulse oximetry manufacturers have developed new algorithms to reduce some false alarms during motion such as extending averaging times or freezing values on the screen, but they do not claim to measure changing conditions during motion and low perfusion. So, there are still important differences in performance of pulse oximeters during challenging conditions.

Published papers have compared signal extraction technology to other pulse oximetry technologies and have demonstrated consistent favorable results for signal extraction technology. Signal extraction technology pulse oximetry performance has also been shown to translate into helping clinicians improve patient outcomes. In one study, retinopathy of prematurity (eye damage) was reduced by $58 \%$ in very low birth weight neonates at a center using signal extraction technology, while there was no decrease in retinopathy of prematurity at another center with the same clinicians using the same protocol but with non-signal extraction technology.

Other studies have shown that signal extraction technology pulse oximetry results in fewer arterial blood gas measurements, faster oxygen weaning time, lower sensor utilization, and lower length of stay.The measurethrough motion and low perfusion capabilities it has also allow it to be used in previously unmonitored areas such as the general floor, where false alarms have plagued conventional pulse oximetry.

In 2011, an expert workgroup recommended newborn screening with pulse oximetry to increase the detection of critical congenital heart disease (CCHD). The CCHD workgroup cited the results of two large, prospective studies of 59,876 subjects that exclusively used signal extraction technology to increase the identification of CCHD with minimal false positives.The CCHD workgroup recommended newborn screening be performed with motion tolerant pulse oximetry that has also been validated in low perfusion conditions. In 2011, the US Secretary of Health and Human Services added pulse oximetry to the recommended uniform screening panel. Before the evidence for screening using signal extraction technology, less than $1 \%$ of newborns in the United States were screened. Today, the Newborn Foundation has documented near universal screening in the United States and international screening is rapidly expanding. In 2014, a third large study of 122, 738 newborns that also exclusively used signal extraction technology showed similar, positive results as the first two large studies.

High resolution pulse oximetry (HRPO) has been developed for in home sleep apnea screening and testing in patients for whom it is impractical to perform polysomnography.It stores and records both pulse rate and $\mathrm{SpO}_{2}$ in 1 second intervals and has been shown in one study to help to detect sleep disordered breathing in surgical patients.

In 1995 Masimo introduced perfusion index, quantifying the amplitude of the peripheral plethysmograph waveform. Perfusion index has been shown to help clinicians predict illness severity and early adverse respiratory outcomes in neonates, predict low superior vena cava flow in very low birth weight infants, provide an early indicator of sympathectomy after epidural anesthesia, and improve detection of critical congenital heart disease in newborns.

In 2007, Masimo introduced the first measurement of the pleth variability index (PVI), which multiple clinical studies have shown provides a new method for automatic, noninvasive assessment of a patient's ability to respond to fluid administration.Appropriate fluid levels are vital to reducing postoperative risks and improving patient outcomes. Fluid volumes that are too low (under-hydration) or too high (overhydration) have been shown to decrease wound healing and increase the risk of infection or cardiac complications.Recently, the National Health Service in the United Kingdom and the French Anesthesia and 
Critical Care Society listed PVI monitoring as part of their suggested strategies for intraoperative fluid administration monitoring.

Perfusion Index or P.I. is the ratio of the pulsatile blood flow to the non-pulsatile static blood flow in a patient's peripheral tissue, such as finger tip, toe, or ear lobe. Perfusion index is an indication of the pulse strength at the sensor site. The P.I.'s values range from $0.02 \%$ for very weak pulse to $20 \%$ for extremely strong pulse. The perfusion index varies depending on patients, physiological conditions, and monitoring sites. Because of this variability, each patient should establish his own "normal" perfusion index for a given location and use this for monitoring purpose.

Perfusion index is normally monitored with pulse oximeters. P.I. is also a good indicator of the reliability of the pulse oximeter reading. For most pulse oximeters for general use, the reading is unreliable or unavailable if P.I. is at or below $0.4 \%$. There are oximeters, such as those from Masimo, designed for extreme low P.I. Most people that use an oximeter at home would not need a perfusion index indicator because they are considered to be in general good health. A perfusion index adds a lot of sensitivity to the oximeter sensors thus adding to the cost of the oximeter.

The pleth (Plethysmograph), available in many pulse oximeters, is a graphical representation of the perfusion index.

In a hospital, perfusion index, along with many other parameters, is used to monitor critically ill patients. Studies have shown that PI has a high correlation with capillary refill time and central-to-toe temperature difference. In neonatal acute care, a low P.I. is an objective and accurate measure of acute illness. It is superior to qualitative approach such as foot warmth.

Perfusion index is also used as an early warning of anesthetic failure. Studies have shown that an increase in PI is an early indicator that general or epidural anesthesia has initiated peripheral blood vessel dilation, which typically occurs before the onset of anaesthesia. Lacking the spike would indicate the lack of anaesthetic effect. Other uses of the perfusion index can be found throughout various literature. As we learn more about P.I., more clinical applications are being discovered. To make informed patient management decision, physicians often need to be aware of changes in perfusion index peripheral perfusion and circulatory status. This is especially true in patients who are in critical conditions or who are anesthetized, undergoing surgery or in labour.The perfusion index (PI) is the ratio of the pulsatile blood flow to the nonpulsatile or static blood in the peripheral tissue .Perfusion index thus represents a non invasive measure of peripheral perfusion that can be continuously and noninvasively obtained from a pulse oximeter.

As the sensitivity of certain pulse oximeter has improved, the fidelity and reliability of PI has improved to a level where clinician are beginning to explore various ways they can utilize PI to care for their patients.

Optimal pulse oximetery monitoring accuracy is dependent on the selection of a monitoring site (fingertip, hand, toe, foot, forehead ear) characterized by good perfusion with oxygenated blood. The PI provides instant and continuous feedback as to the perfusion status of the selected monitoring site.In clinical scenarios where peripheral perfusion may drop below the minimum required for the tissue oxygenation and cellular respiration, the PI alerts the clinician to consider another monitoring sites. Optimal monitoring sites are chosen with a relatively high stable PI.

Clinical studies in adults and paediatric patients have demonstrated that an increase in P.I. is an early indication that general and epidural anesthesia has initiated peripheral vasodilation which typically occurs before the onset of the anesthetic effects. Detection of a spike in PI is a sign to the physician of the successful onset of anesthesia and absence of it may be an early warning of anesthetic failure. As an objective indicator of pain levels in patients the PI has been used to determine proper management of pain, especially in patients unable to communicate their discomforts to the clinician.

In the neonatal acute care setting, a low perfusion index has been shown to be an objective and accurate measure of acute illness. The determinationof the perfusion index is an umanbiguous and independent parameter compared to subjective means of assessing health status in the neonates.Additionally, perfusion index measurement represents a more rapid and inexpensive method to access peripheral perfusion and circulatory status in neonates. Additionally, perfusion index measurement represents a more rapid and inexpensive method to access peripheral perfusion and circulatory status in comparision to evaluating calf muscle perfusion and oxygen consumption by way of near infrared spectroscopy. 
In the neonatal acute setting, a low PI has been shown to be an objective and accurate measure of acute illness .The determination of PI is unambiguous and independent compared to subjective means of assessing health status in neonates.Additionally, PI measurement represents a more rapid and inexpensive methods to assess peripheral perfusion and circulatory status in comparision to evaluating calf muscle perfusion and oxygen consumption.

PI monitoring warrants further exploration for other clnical applications were information on peripheral perfusion or circulatory status would be useful. Potential future applications include prediction of the success of reimplanted body parts, restoration of peripheral perfusion after cardiopulmonary bypass and estimation of volume status in trauma patients.

\section{CLINICAL INTERPRETATION OF PERFUSION INDEX}

PERFUSION INDEX is an assessment of the pulsatile strength at a specific monitoring site,(e.g. the finger tip or toe), and as such P.I. is an indirect and noninvasive measure of peripheral perfusion .It is calculated by means of pulse oximetery by expressing the pulsatile signal (during arterial inflow)as a percentage of the non pulsatile signal, both of which are derived from the amount of infrared (940nm) light absorbed.

The P.I. value is relative to a particular monitoring site, (e.g. the fingertip or toe), of each patient as physiological conditions vary between monitoring sites and indivisual patients.MASIMO signal extraction technology pulse oximetery yields a a continual and simultaneous absolute values and trends with associated alarms for PI, arterial oxygen saturation and pulse rate using validated signal extraction technology.because SET technology utilize five signal processing algorithm to deliver high precision sensitivity and specificity in the measurement of blood oxygen saturation levels, the P.I. parameter can be derived from the core measurements of SET and yields clinically useful information regarding the peripheral status of the patients .Other indices of perfusion index derived through infrared absorption data lack the sensitivity technology of SET.

Ability to trend the perfusion index is critical .Only the trend will show the subtle changes in perfusion that is otherwise missed in static displays. These subtle changes captured by the trend provides immediate clinical feedback of the efficacy of anesthesia, analgesia and/ or therapeutic intervention. Integrating user formatable alarms with P.I. provide clinician with immediate feedback to increase or decreases of P.I. to gain optimal results in the clinical management of patients.

Changes in PI can also occur as results of local vasoconstriction (a decrease in PERFUSION INDEX) or vasodilatation (increase in perfusion index) in the skin in monitoring site. These changes occur with changes in the volume of oxygenated blood flow in the skin microvasculature. The measurement of PI is independent of other physiological variables such as heart rate variability, $\mathrm{SPO}_{2}$, oxygen consumption or temperature. Interpretation of P.I. depends upon the clinical context in which it is applied. The P.I. generally changes in proportion to the peripheral perfusion.

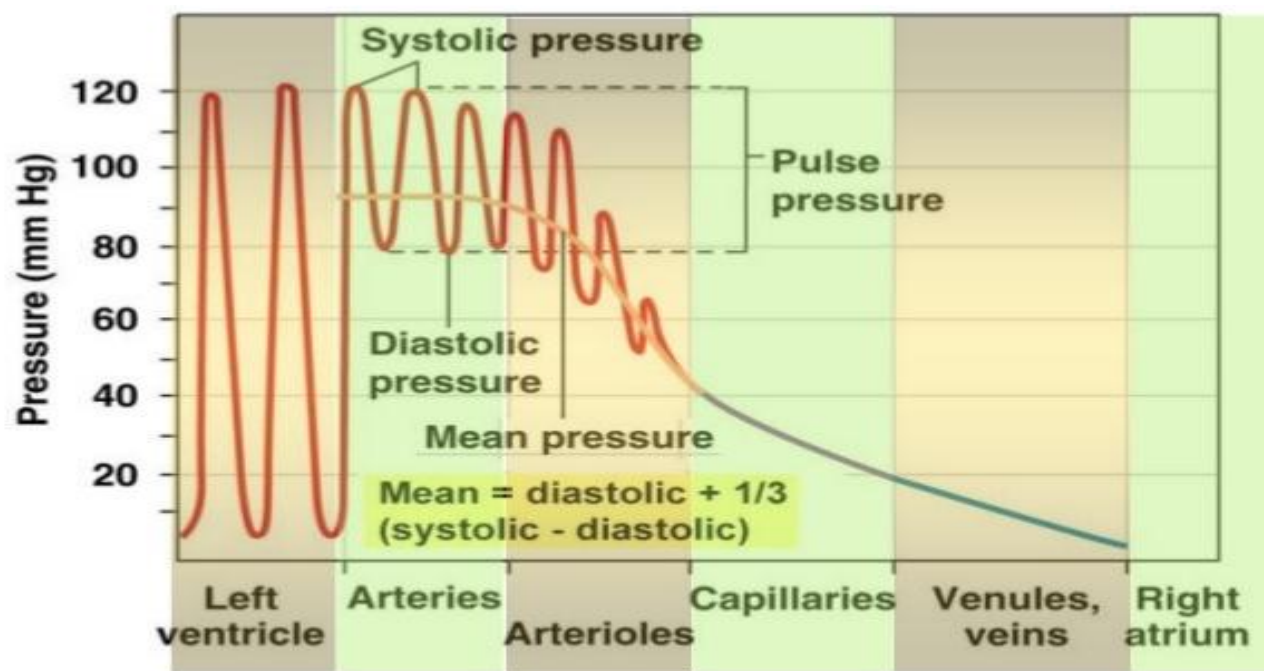

Figure No. 1: Arterial Blood Flow Waveform in Large Vessel, Small Vessel, Arterioles and Capillaries 


\section{Functions of BP}

- Intraventricular BP $\rightarrow$ ejection of blood (stroke volume)

- Systemic arterial BP $\rightarrow$ blood flow to tissues (tissue perfusion)

- Capillary hydrostatic pressure $\rightarrow$ filtration (tissue fluid formation)

- Systemic venous BP $\rightarrow$ blood flow back to the heart (venous return)

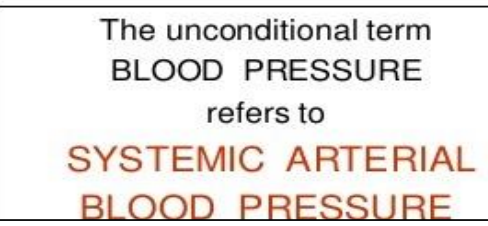

\section{Systemic arterial blood pressure $=$ Cardiac output $\times$ Total peripheral resistance (TPR) or Systemic vascular reistance (SVR)}

The 2 Major Determinants of Arterial B.P.

\section{The other Determinants of Arterial B.P.?}

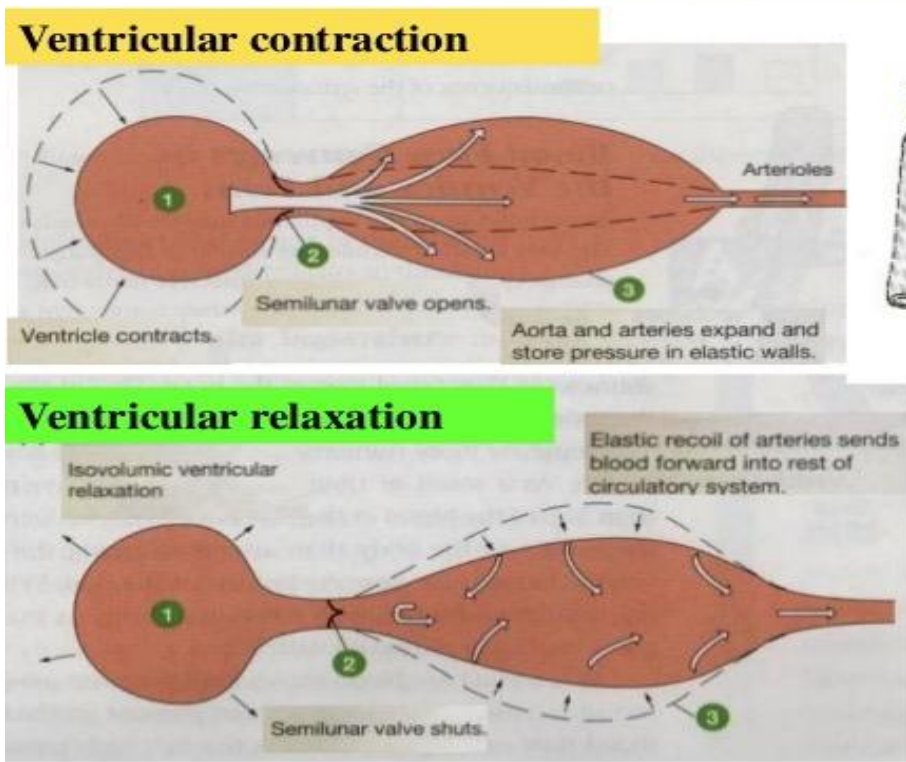

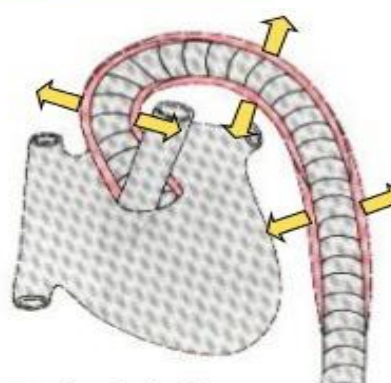

Aorta is the most elastic artery

When aortic elasticity decreases (ageing or disease):

Less expansion during systole $\rightarrow$ Incr. SBP

Less elastic recoil during diastole $\rightarrow$ decr. DBP

Pulse pressure? 
The Determinants of Arterial B.P.

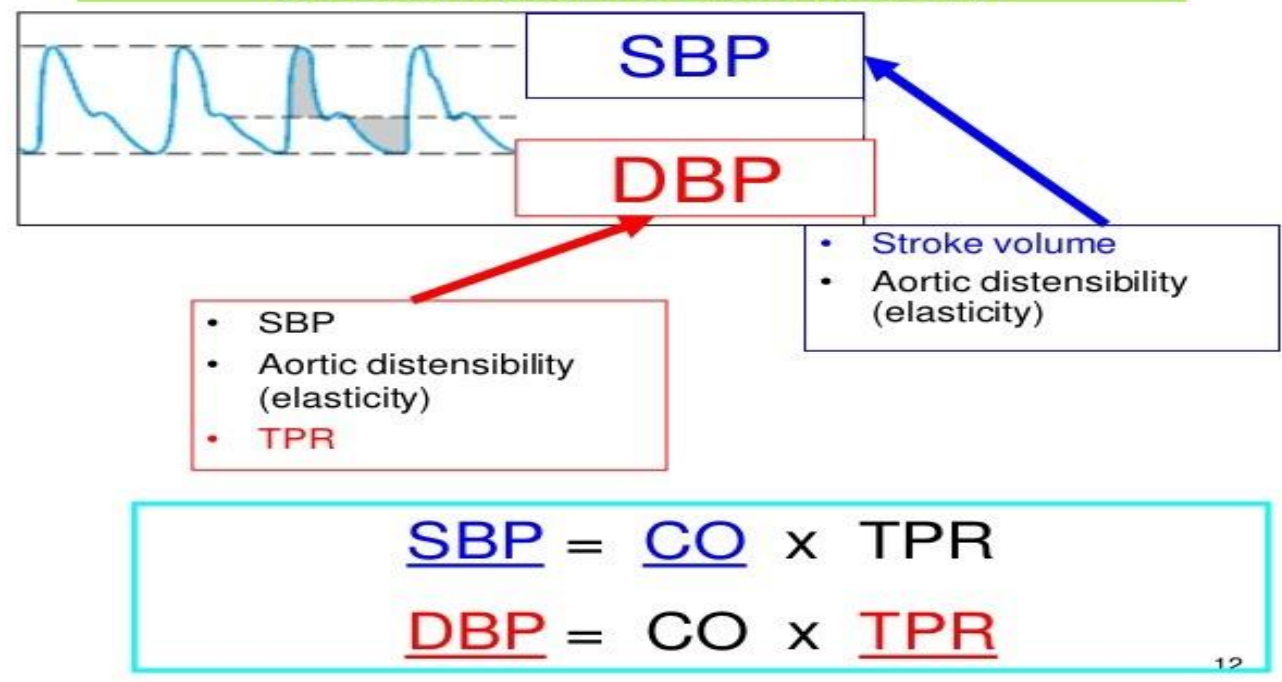

Figure No. 2: Blood Pressure during Systole and Diastole

\section{Physiological Variations in BP}

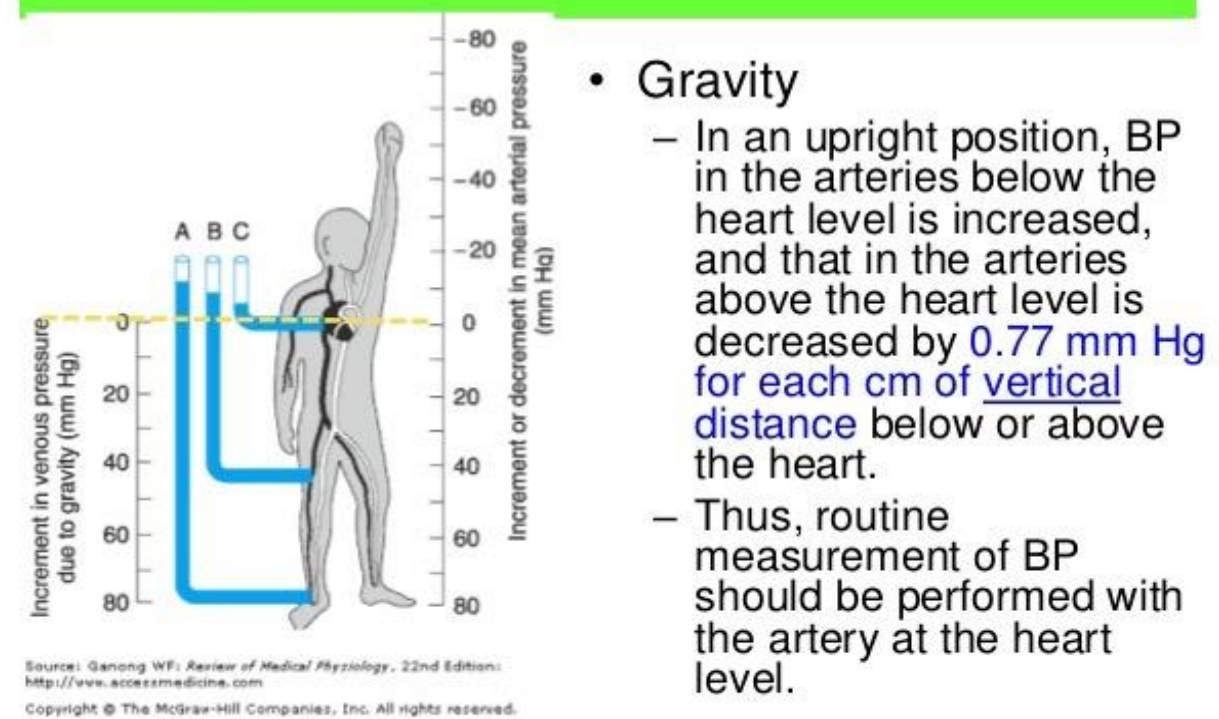

Figure No. 3: Physiological Variation in Blood Pressure 


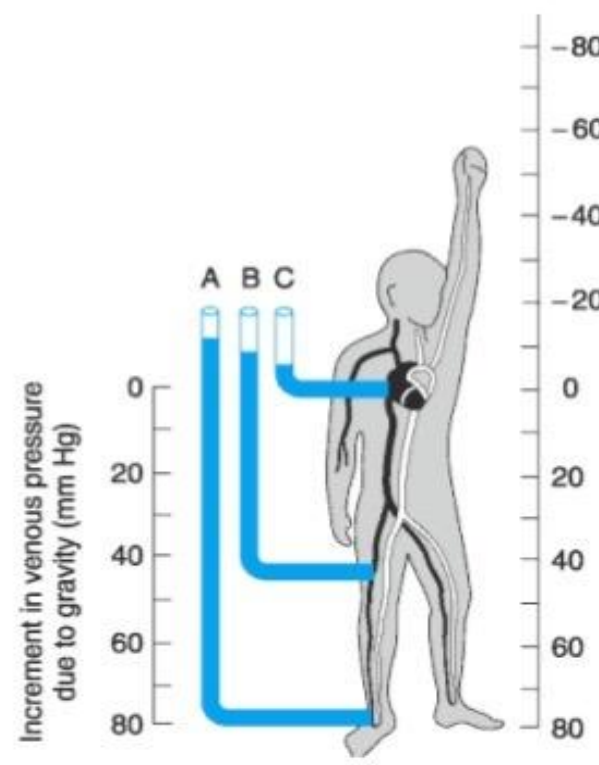

Source: Ganong WFi Review of Madical Phrsiology, 22nd Edition hetp i//vwr. accessmedieine. corm

Copyright $\theta$ The Mcaraw-Mill Companies, Ine All rights reserved.

\section{Effect of Gravity}

- Pressure in large artery in the foot $105 \mathrm{~cm}$ below the heart $=$ $[0.77 \mathrm{mmHg} / \mathrm{cm} \times 105 \mathrm{~cm}=80$ $\mathrm{mm} \mathrm{Hg})]+$

$100 \mathrm{~mm} \mathrm{Hg}$ (Mean ABP at heart level)

- $=180 \mathrm{~mm} \mathrm{Hg}$

- Pressure in vein in the foot 105 $\mathrm{cm}$ below the heart $=[0.77$ $\mathrm{mmHg} / \mathrm{cm} \times 105 \mathrm{~cm}=80 \mathrm{~mm}$ $\mathrm{Hg})]+$

- $4 \mathrm{~mm} \mathrm{Hg}$ (right atrial pressure)

- $=84 \mathrm{~mm} \mathrm{Hg}$

Systemic arterial blood pressure $=$ Cardiac output $\mathrm{x}$

Total peripheral resistance (arteriolar tone)

More immediate

More efficient:

$$
\text { RESISTANCE }=\frac{1}{\text { Radius } \mathbf{4}}
$$

More economical

Figure No. 4: Physiological Variation in Blood Pressure: Effect Of Gravity 


\section{How does sympathetic N.S. activity increase BP?}

- Direct cardiovascular effects

- Neuroendocrine effects: activation of

- adrenal medulla

- renin-angiotensin-aldosterone (RAA) system

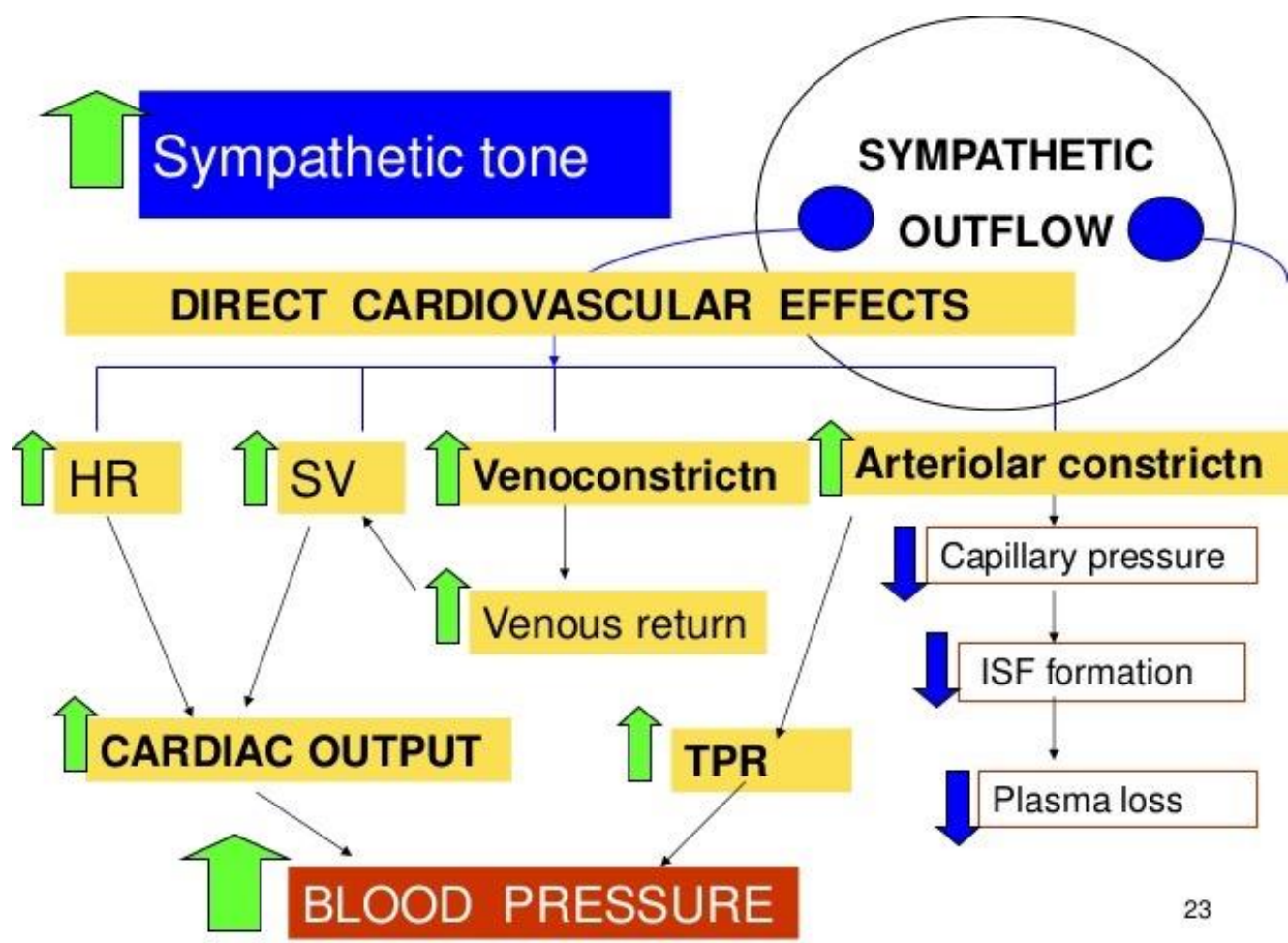

Figure No. 5: Sympathetic Tone and Blood Pressure 


\section{RENIN- ANGIOTENSIN-ALDOSTERONE SYSTEM}
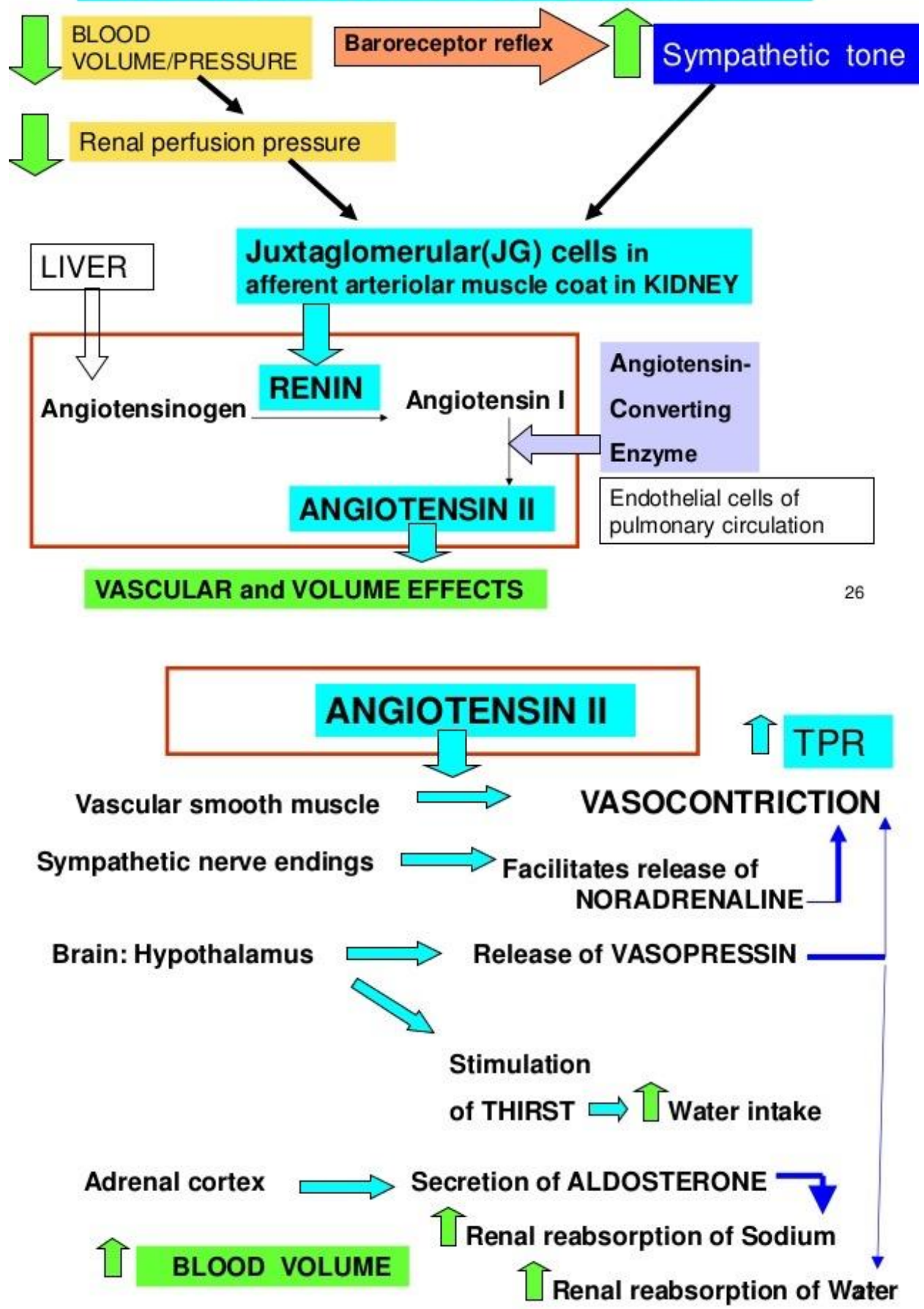

Figure No. 6: Renin- Angiotensin- Aldosterone System 


\section{Arterial baroreceptors}

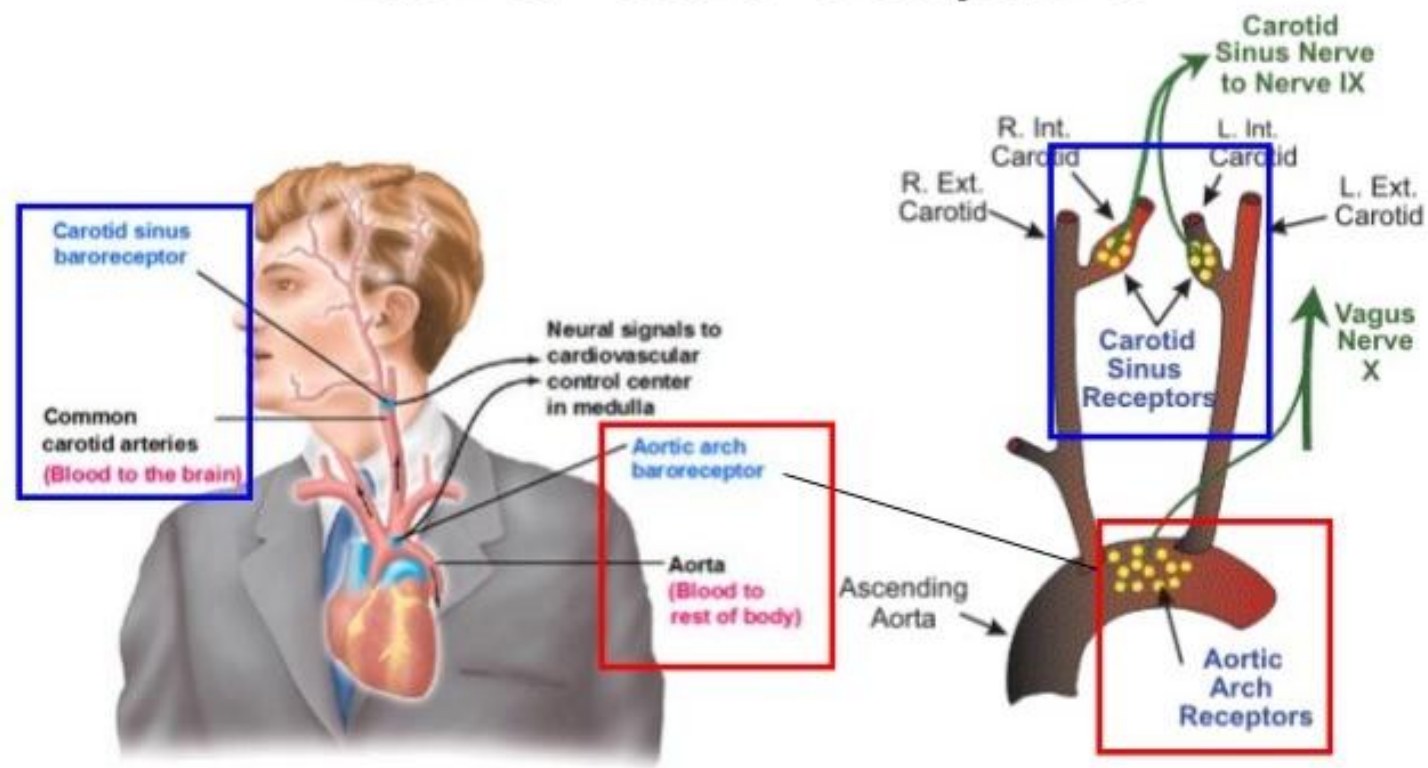

Figure No 7: Arterial Baroreceptors

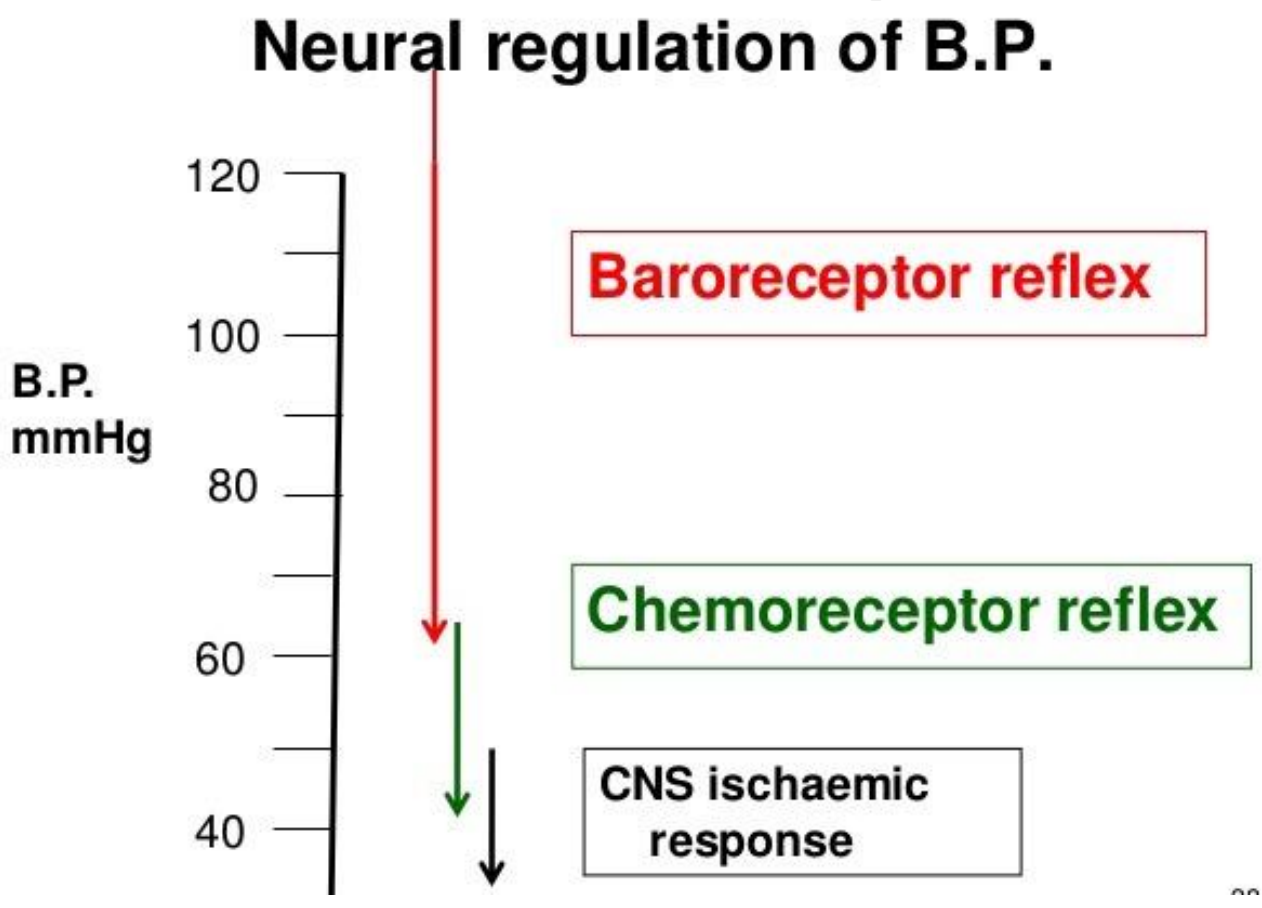




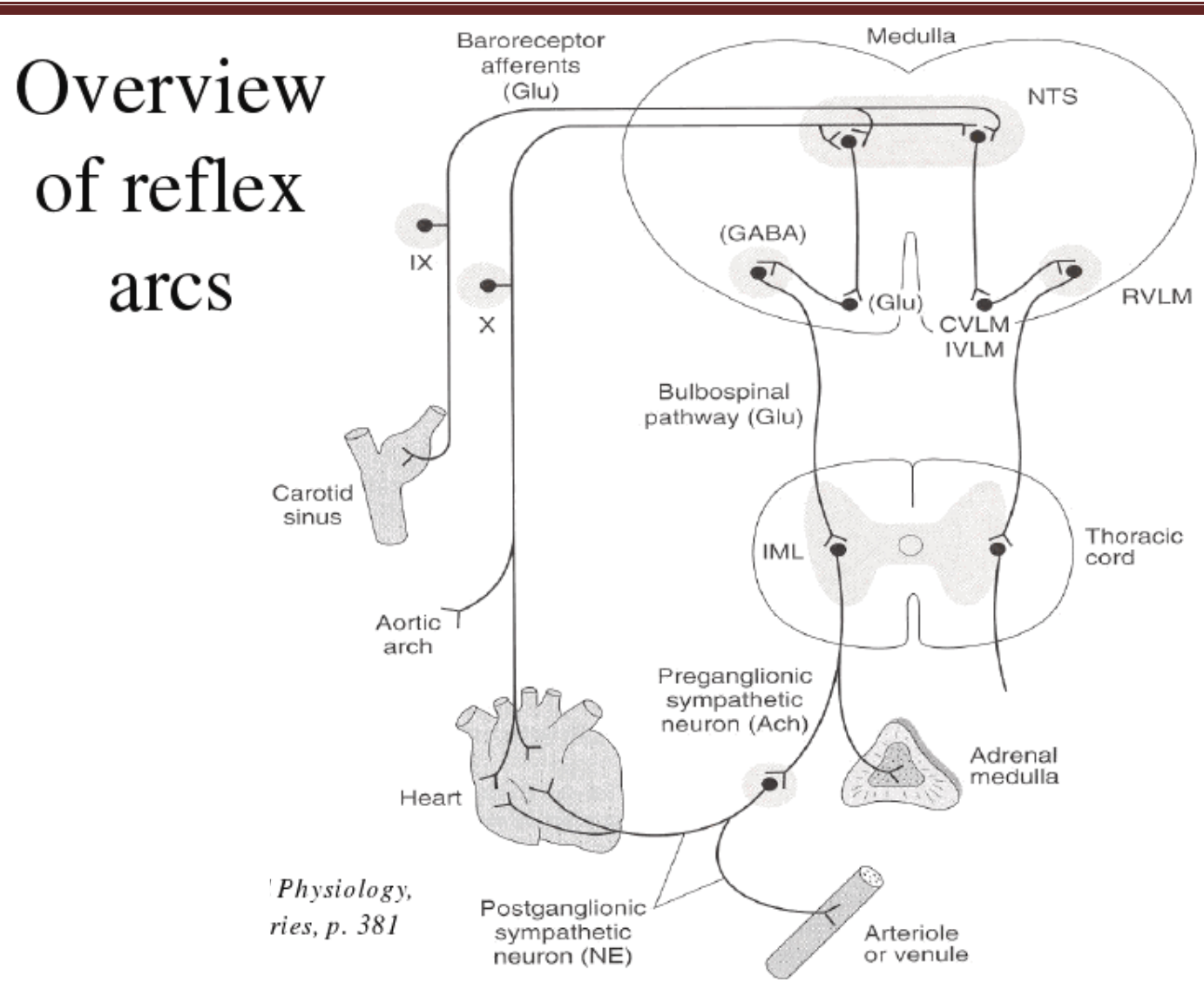

Figure No. 8: Arterial Chemoreflex and Baro Reflex

Heart rate is normally determined by the pacemaker activity of the sinoatrial node (SA node) located in the posterior wall of the right atrium. The SA node exhibits automaticity that is determined by spontaneous changes in $\mathrm{Ca}++, \mathrm{Na}+$, and $\mathrm{K}+$ conductances. This intrinsic automaticity, if left unmodified by neurohumoral factors, exhibits a spontaneous firing rate of 100-115 beats/min. This intrinsic firing rate decreases with age.

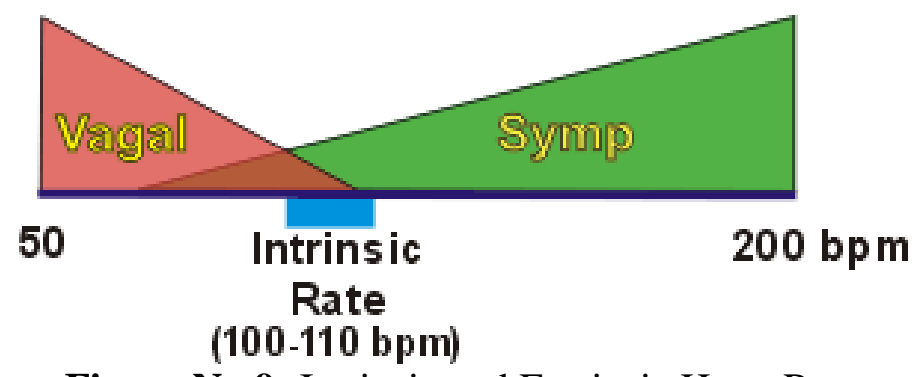

Figure No 9: Intrinsic and Extrinsic Heart Rate

Heart rate is decreased below the intrinsic rate primarily by activation of the vagus nerve innervating the SA node. Normally, at rest, there is significant vagal tone on the SA node so that the resting heart rate is between 60 and 80 beats/min. This vagal influence can be demonstrated by administration of atropine, a muscarinic receptor antagonist, which leads to a 20-40 beats/min increase in heart rate depending upon the initial level of vagal tone.

For heart rate to increase above the intrinsic rate, there is both a withdrawal of vagal tone and an activation of sympathetic nerves innervating the SA node. This reciprocal change in sympathetic and parasympathetic activity permits heart rate to increase during exercise, for example.

Heart rate is also modified by circulating catecholamines acting via $\beta 1$-adrenoceptors located on SA nodal cells. Heart rate is also modified by changes in circulating thyroxin (thyrotoxicosis causes tachycardia) and by changes in body core temperature (hyperthermia increases heart rate).

SA nodal dysfunction can lead to sinus bradycardia, sinus tachycardia, or sick-sinus syndrome. 
The maximal heart rate that can be achieved in an individual is estimated by Maximal Heart Rate $=220$ beats/min - age in years Therefore a 20 -year-old person will have a maximal heart rate of about 200 beats/min, and this will decrease to about 170 beats/min when the person is 50 years of age. This maximal heart rate is genetically determined and cannot be modified by exercise training or by external factors Perfusion Index (PI)

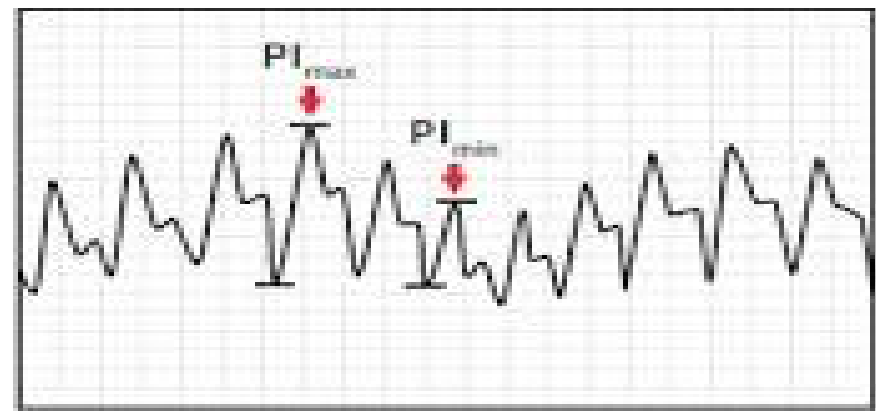

Figure No. 10: Perfusion Index Is Quantifiucation of Amplitude Of Plethysmograph

\section{What is PI?}

- Perfusion Index, or PI, is a relative assessment of the pulse strength at the monitoring site.

- PI display ranges from .02\% (very weak pulse strength) to $20 \%$ (very strong pulse strength).

- Perfusion Index is a numerical value that indicates the strength of the IR (infrared) signal return- ing from the monitoring site.

- The LED-based Masimo pulse oximeters such as Rad-5 and Rad-5v display PI as the Pulse Amplitude Index (PAI) where the height of the bar represents the pulse strength. Low PAI is indicated by the lower two bar segments turning red.

- PI is a relative number and varies between monitoring sites and from patient to patient, as physiologic conditions vary.

\section{How do I use PI in a clinical setting?}

- During sensor placement, use PI to quickly evaluate the appropriateness of an application site, looking for the site with the highest P.I. number.

- Placing the sensor at the site with the strongest pulse amplitude (highest P.I number) improves performance during motion. Monitor the trend of the PI for changes in physiologic conditions.

- Changes in sympathetic nervous tone affect smooth muscle tone, thereby altering levels of perfusion.

\section{How does PI work?}

- The Masimo SET infrared signal is influenced primarily by the amount of blood at the monitoring site, not by the level of oxygenation in the blood.

- The ratio of AC (pulsatile) to DC (non-pulsatile) components of the IR (infrared) signal corre- spond to the pulsatile and the non-pulsatile amounts of blood.

- The relationship of the pulsatile to the non-pulsatile amounts of blood at any particular site corresponds to PI at that site.

\section{Has PI been clinically validated?}

- Preliminary data suggest high PI values correspond with health and low PI values with illness on neonates.

- Clinical Applications of Perfusion Index

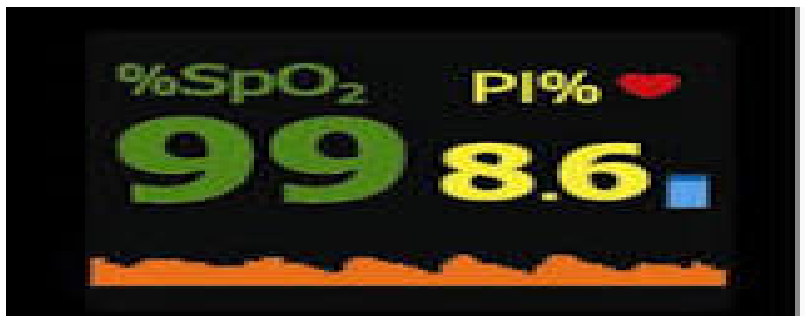




\section{Figure No. 11: Perfusion Index with Spo2 Monitoring with Plethysmograph Below}

To make informed patient management decisions, physicians often need to be aware of changes in peripheral perfusion and circulatory status. This is especially true in patients who are in critical condition, or who are anesthetized, undergoing surgery, or in labor. The perfusion index (PI) is the ratio of the pulsatile blood flow to the nonpulsatile or static blood in peripheral tissue. Perfusion Index thus represents a noninvasive measure of peripheral perfusion that can be continuously and noninvasively obtained from a pulse oximeter.

As the sensitivity of certain pulse oximeters has improved, the fidelity and reliability of PI has improved to a level where clinicians are beginning to explore various ways they can utilize PI to care for their patientsOptimal pulse oximetry monitoring accuracy is dependent on the selection of a monitoring site (fingertip, hand, toe, foot, forehead, nose, ear) characterized by good perfusion with oxygenated blood. The PI provides instant and continuous feedback as to the perfusion status of the selected monitoring site. In clinical scenarios where peripheral perfusion may drop below the minimums required for tissue oxygenation and cellular respiration, the PI alerts the clinician to consider another monitoring site. Optimal monitoring sites are chosen with a relatively high, stable PI.

\section{Clinical Interpretation of the Perfusion Index}

Perfusion index is an assessment of the pulsatile strength at a specific monitoring site (eg.the hand, finger or foot), and as such PI is an indirect and noninvasive measure of peripheral perfusion. It is calculated by means of pulse oximetry by expressing the pulsatile signal (during arterial inflow) as a percentage of the nonpulsatile signal, both of which are derived from the amount of infrared $(940 \mathrm{~nm})$ light absorbed.

The PI value is relative to a particular monitoring site, (eg. the fingertip or toe), of each patient as physiological conditions vary between monitoring sites and individual patients. Masimo SET pulse oximetry yields continual and simultaneous absolute values and trends with associated alarms for PI, arterial oxygen saturation $\left(\mathrm{SaO}_{2}\right)$, and pulse rate using validated signal extraction technology. Because SET technology has sensitivity ratings proven to be the industry standard, the PI parameter yields clinically useful information. Other indices of perfusion derived through infrared absorption data lack the sensitivity technology of SET, limiting the power of the index.

The ability to trend the P.I. is critical; only the trend reveals the often subtle changes in perfusion that are otherwise missed by static displays. These subtle changes captured by the trend provide immediate clinical feedback of the efficacy of anesthesia, analgesia, and/or therapeutic intervention. Integrating user-formatable alarms with PI provides clinicians with immediate feedback to increases or decreases of PI to gain optimal results in the clinical management of the patient.

Changes in P.I. can also occur as a result of local vasoconstriction (decrease in P.I.) or vasodilatation (increase in P.I.) in the skin at the monitoring site. These changes occur with changes in the volume of oxygenated blood flow in the skin microvasculature. The measurement of P.I. is independent of other physiological variables such as heart rate variability, $\mathrm{SaO}_{2}$, oxygen consumption, or temperature.

The interpretation of P.I. depends on the clinical context to which it is applied. The PI generally changes in proportion to peripheral perfusion. In certain instances, however, such as in a patient attached to a heart-lung machine, perfusion can be good but the pulsatile part of the signal is nearly zero because of the absence of a pulse. Even in such an instance, the monitoring of P.I. in conjunction with examining the photoplethysmogram (pleth) waveform, can give the clinician an indication of the accuracy of the saturation readings.

\section{Choosing a Monitoring Site in Adults}

The P.I. is useful for quickly evaluating the appropriateness of a monitoring site for pulse oximetry. A site with high pulse amplitude (high P.I. number) generally indicates an optimal monitoring site for other pulse oximetry and Pulse CO-Oximetry measures. The fingertip is the standard monitoring site for pulse oximetry. The hand or foot (sometimes toe) is often used in neonatal patients. Surgical patients, however, are subject to unpredictable changes in peripheral perfusion, particularly with a large degree of variability in body temperature. Such changesin peripheral perfusion may have variable effects at different sensor locations. It is useful, therefore, to have an alternative to the standard fingertip sensor site. 
Monitoring Perfusion Index in Anesthetized Patients

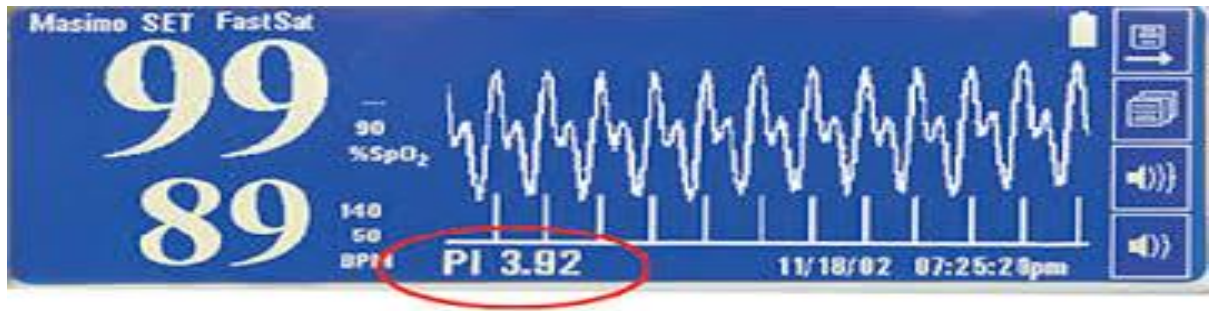

Figure No. 12: Perfusion Index As Seen In Masima Set Fastsat Monitor

Most anesthetics produce a vasodilatative effect by way of increasing the vasodilatation threshold and decreasing the vasoconstriction threshold. Anesthesia can also cause temperaturere distribution, which further effects peripheral perfusion. Perfusion index has been considereda useful tool for accurately monitoring changes in peripheral perfusion in real time caused by certainanesthetics. Masimo SET pulse oximetry was usedto monitor P.I. in a study of 7 patients undergoing major abdominal surgery.In this study, the P.I.showed a statistically significant correlation with end-expiratory sevoflurane $(R=0.005, p<0.001)$. In contrast, the more conventional forearm-finger tip gradient did not correlate with either sevoflurane concentration $(\mathrm{R}=0.05, \mathrm{p}=0.5)$ or PI $(\mathrm{R}=0.22, \mathrm{p}=0.15)$. These results suggests the future value of considering perioperative changes in P.I. during surgical procedures to monitor temperature redistribution, vasodilatation, and the efficacy of anaesthesia.

A prospective study was carried out in 101 neonates to evaluate the relationship between illness severity and P.I. Perfusion Index, $\mathrm{SpO}_{2}$, and heart rate were monitored using the Masimo SET Radical pulse oximeter.

A total of 43 neonates fit criteria for inclusion into the high severity group and 58 neonates met low severity group criteria (stratified based on SNAP II scores). The 2 groups were similar in terms of sex, gestational age, birth weight, body temperature, mean blood pressure, and use of peripheral vasoconstrictors and vasodilators. Significantly lower P.I. values $(0.86 \pm 0.26$ vs. $2.02 \pm 0.70, \mathrm{p}<0.0001), \mathrm{SpO} 2(93.3 \pm 5.4 \%$ vs. $95.1 \pm 3.9 \%, \mathrm{p}<0.0001)$, and higher pulse rate $(139 \pm 16 \mathrm{bpm}$ vs. $133 \pm 17 \mathrm{bpm}, \mathrm{p}<0.0001)$ were found in the high severity group.

The authors concluded a foot skin P.I. value of $\leq 1.24$ is an unambiguous and accurate predictor of illness severity and is independent of subjective means of interpreting neonatal health status. These authors also demonstrated that when used in conjunction with oxygen saturation and pulse rate, a diminished PI becomes an important indicator of chorioamnionitis (HCA) in term newborns - a condition that is often subclinical and associated with neonatal morbidity and mortality.

Simple bedside clinical tool for evaluating circulatory status in neonates that could become a standard for neonatal intensive care.

The perfusion index is an indirect, noninvasive, and continuous measure of peripheral perfusion that provides useful information to the practicing physician in several clinical settings. Pulse oximetry provides a relatively simple means to continuously monitor P.I. in conjunction with other critical parameters, i.e., oxygen saturation and pulse rate. Furthermore, the P.I. provides a means of determining an appropriate monitoring site for pulse oximetry.

Potential future applications for monitoring perfusion index:

Indication of circulatory function of re- implanted body parts such as fingers or hands.

Restoration of peripheral perfusion after cardiopulmonary bypass

- Estimation of volume status in trauma patients.

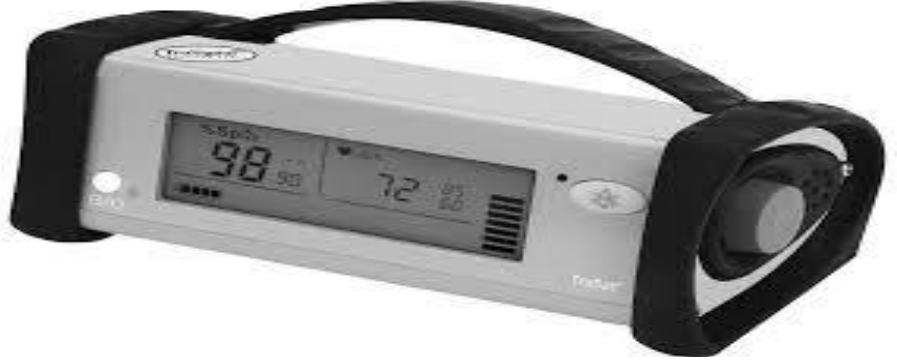

Figure No 13: GE Ohmeda Tru Sat Tm Model: Gtm21089-1512-T3 (This apparatus was used in study) 
In the neonatal acute care setting, a low PI has been shown to be an objective indicator of severe illness. In conjunction with oxygen saturation and pulse rate, a diminished P.I. becomes an important indicator of a critical state of neonatal health. As such, the P.I. may be important to consider as a standardized, objective measure in addition to conventional subjective means of assessing the state of the neonate.

P.I. determination has emerged as an important bedside diagnostic and monitoring tool with applications in multiple clinical settings. Advancements in signal processing and P.I. features have aided clinicians in further utilizing this valuable measurement. The ability to trend and set alarms for P.I. changes are now available in Masimo SET devices. Only the trend reveals the often subtle changes in perfusion that are otherwise missed by static displays. Integrating user formatable alarms with P.I. provide clinicians with immediate feedback to increases or decreases in PI. With further use and study, P.I. is likely to prove useful for evaluating patient outcomes and monitoring progress in other situations when peripheral perfusion and circulatory status should be evaluated.

\section{Pulse oximeter pleth (plethysmograph)}

Pulse oximeter can measure our $\mathrm{SpO}_{2}$ and blood flow fluctuations.A plethysmograph is an instrument for measuring changes in volume within an organ or whole body (usually resulting from fluctuations in the amount of blood or air it contains). A photoplethysmograph (PPG) is a plethysmograph that uses optical techniques. A pulse oximeter measures oxygen saturation level $\left(\mathrm{SpO}_{2}\right)$ and is also a PPG. It can measure the change in the volume of arterial blood with each pulse beat. This change in blood volume can be detected in peripheral parts of the body such as the fingertip or ear lobe using a technique called photoplethysmography. The pulse oximeter that detects the signal is called a plethysmograph (or 'Pleth' for short).

Because of the nature of pulse oximeter, measuring the pressure from a pleth waveform has not been possible. However, the information has shown to be useful in diagnosis and in gauging the severity of illness in newborns. Many doctors use the pulse oximeter's pleth waveform as an early indication for cyclic changes in physiology. If the variability increases, it indicates a change in the intrathoracic blood volume relationship.

The blood flow is normally shown as a waveform using a bar or graph. It can provide useful information regarding the heart condition.

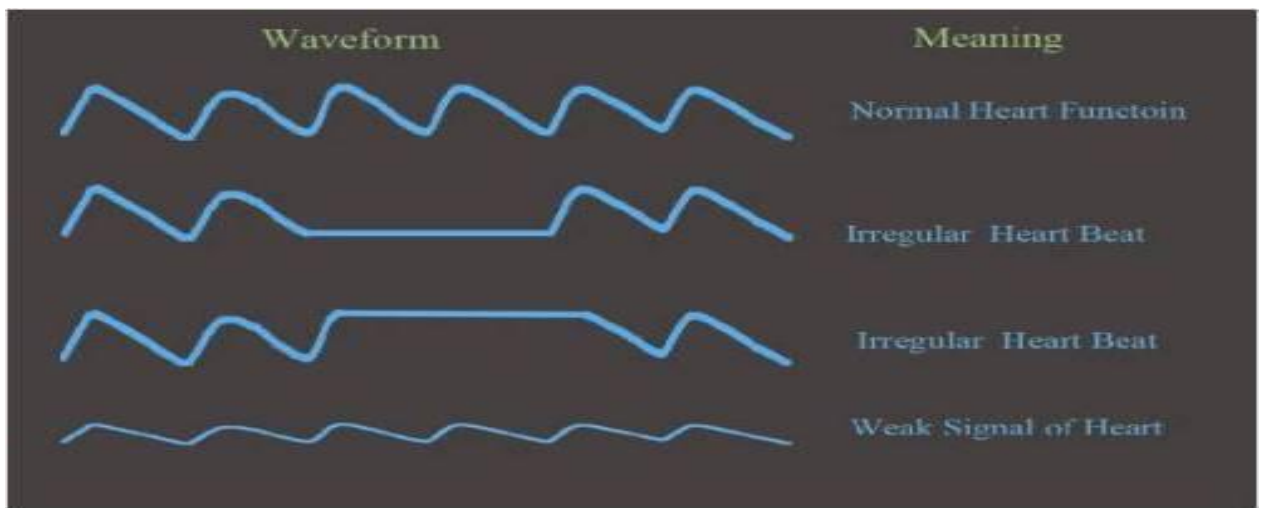

Figure No. 14: the following diagram illustrates the relationship among pleth (PPG), premature ventricular contraction (PVC), blood pressure (B.P.), and EKG.

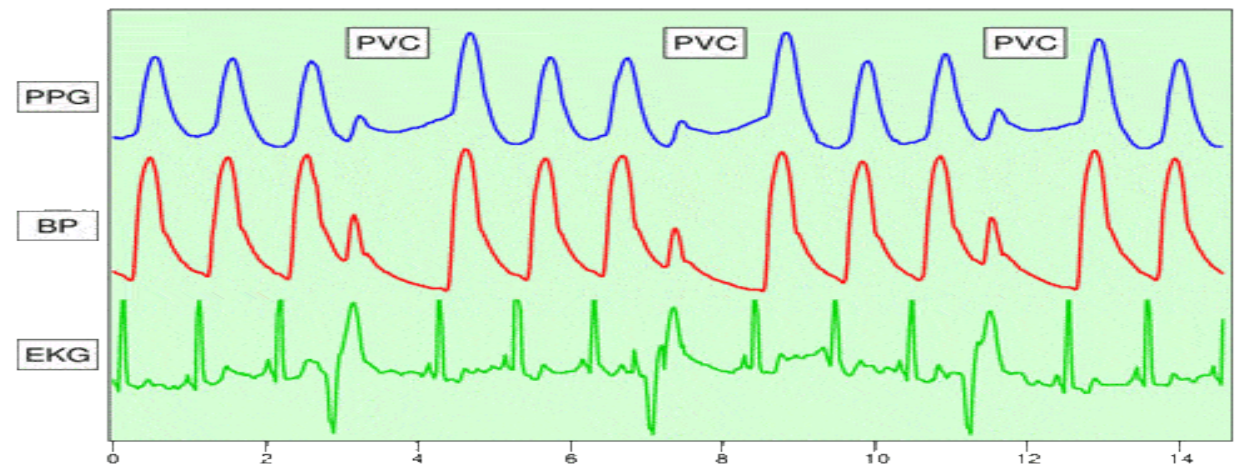

Figure No. 15: Plethysmograph in abnormal heart rate rhythm 
Heart rhythm. Pleth is useful to detect irregular heart rhythm. The primary function of the heart is to supply blood and nutrients to the body. The regular beating, or contraction, of the heart moves the blood throughout the body. By watching the pleth waveform, the heart beating pattern is clearly displayed. When a heart patient is having discomfort, it is important to observe the pleth waveform and relate the information to his doctor. Since the discomfort is generally transient, a doctor may not be able to see the waveform during an office visit.

Signal strength. Weak signal is indicated by the amplitude of the waveform. If the signal is too low, it would affect the accuracy and functioning of the pulse oximeter. If your oximeter is not giving the correct result, check if the signal strength is too low.

There are several causes for the weak signal:

- Low blood perfusion

- Dirty sensors or LED lights

- Improper positioning of the oximeter

Low blood perfusion can be caused by cold temperature and the person's general health.

Respiration During inspiration, intrapleural pressure decreases by up to $4 \mathrm{~mm} \mathrm{Hg}$ which distends the right atrium, allowing for faster filling from the vena cava, increasing ventricular preload, and increasing the stroke volume. Conversely during expiration, the heart is compressed, decreasing cardiac efficiency and reducing stroke volume. However, the overall net effect of respiration is to act as pump for the cardiovascular system. When the frequency and depth of respiration increases the venous return increase, leading to increased cardiac output.

Respiration effects the cardiac cycle by varying the intrapleural pressure, the pressure between the thoracic wall and the lungs. Since the heart resides in the thoracic cavity between the lungs, the partial pressure created by inhaling and exhaling affects the pressure on the heart and hence the blood flow volume.

During inspiration, intrapleural pressure decreases by up to $4 \mathrm{~mm} \mathrm{Hg}$ which distends the right atrium, allowing for faster filling from the vena cava, increasing ventricular preload, and increasing the stroke volume. Conversely during expiration, the heart is compressed, decreasing cardiac efficiency and reducing stroke volume. However, the overall net effect of respiration is to act as pump for the cardiovascular system. When the frequency and depth of respiration increases, the venous return increase leading to increased cardiac output. Pulse oximetry, promptly accepted as a standard for care in the intensive care unit, allows measurement of arterial oxygen saturation and indirect assessment of oxygen transport balance. Indeed, 70 years ago, Karl Matthes developed the first nonpulsatile device that would non- invasively and continuously monitor oxygen saturation. In 1972, Aoyagi invented the conventional pulse oximetry using the ratio of red and infrared light absorption of pulsating components at the measuring site (commonly the finger). Utilizing the optical density ratio, the arterial oxygen saturation $(\mathrm{SpO} 2)$ value was reported. The device combined spectrophotometery and a derived photo- plethysmography (PPG) based on the cyclic variations in blood volumes. Indeed, in addition to the digital read out of oxygen saturation, most pulse oximeters display photoplethysmographic waveforms that permit dis- tinguishing artefactual signal and reject it from the final data computation. A sharp waveform with a clear dicrotic notch indicates good perfusion, whereas a sinewave contour suggests poor perfusion. Photoplethysmography With each heartbeat, the ventricle pumps blood to the periphery and the pulse pressure distends the arteries and arterioles in the subcutaneous tissue. The concept of PPG is based on the illumination of skin and measurements of changes in light absorption . Indeed, the change in volume caused by the pulse pressure is detected after illuminating the skin using a light-emitting diode and measuring the amount of light either transmitted or reflected to a photodiode. The amount of haemoglobin present in the fingertip is abruptly increased and light absorption is decreased during systole, whereas the inverse phenomenon is observed during diastole. The pulse wave- form is then derived from the infrared signal, which is influenced mainly, but not exclusively, by arterial blood. Each pulse then appears as a peak simultaneous to the arterial pulse pressure curve displayed from a radial artery. PPG can be obtained from transmissive absorption (as at the finger tip) or reflective (as on the forehead). The shape of the PPG waveform differs from subject to subject and varies with the location and the manner in which the pulse oximeter is attached. The height of pulse component of the PPG is proportional to the pulse pressure. 
Plethysmographic curve can be used to assess the macro circulation A conventional pulse oximeter monitors the perfusion of blood to the dermis and subcutaneous tissue of the skin. Thus, PPG of the pulse oximeter has been proposed as a method of monitoring macrocirculation and microcirculation. Oximeters have been shown to be useful in detecting systolic blood pressure. On the contrary, some works proposed this method to assess the patency of arterial grafts, the viability of bowel and the early detection of radial artery occlusion owing to arterial lines. The signal of pulse oximetery curves, however, may be lost in the presence of cardiac output less than $2.4 \mathrm{l} / \mathrm{min} / \mathrm{m} 2$ and very high mean systemic vascular resistance index. In patients with severe tricuspid regurgitation, the measured curve may be not related to systolic pressure as venous flow becomes pulsatile. In one report, the PPG amplitude has been shown to increase following arterial vasodilatation, which enhances the respiratory variation of the waveform.

Since many years, several publications have suggested that the PPG waveform contains other underrated informations that is the respiratory variations factor of the waveform . Despite being related to relative change in blood volume in an undersized region of tissue, the PPG signal resembles the peripheral arterial pressure waveform . Indeed, by reflecting the pulsatile changes in absorption of light between the beam source and the photodetector of the pulse oximeter, the 'pulse'wave is assumed to be the result of the beatto-beat changes in stroke volume transmitted to the peripheral circulation. In this regard, analysis of the respiratory variation in the plethysmographic signal measured from pulse oximetry has been proposed for a long time as a technique to assess haemodynamic monitoring in paediatric patients and blood volume status in mechanically ventilated patients .

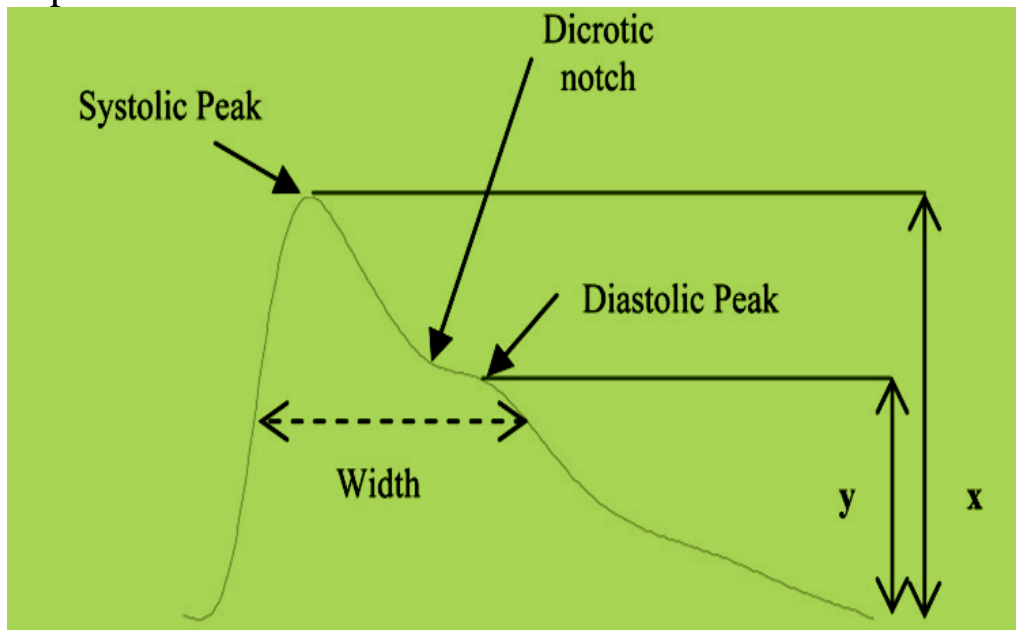

Figure No. 16: A Typical Photoplethysmograph and Its Components

A typical waveform of the PPG and its characteristic parameters, whereas the amplitude of the systolic peaks is $\mathrm{x}$ while $\mathrm{y}$ is the amplitude of the diastolic peak.

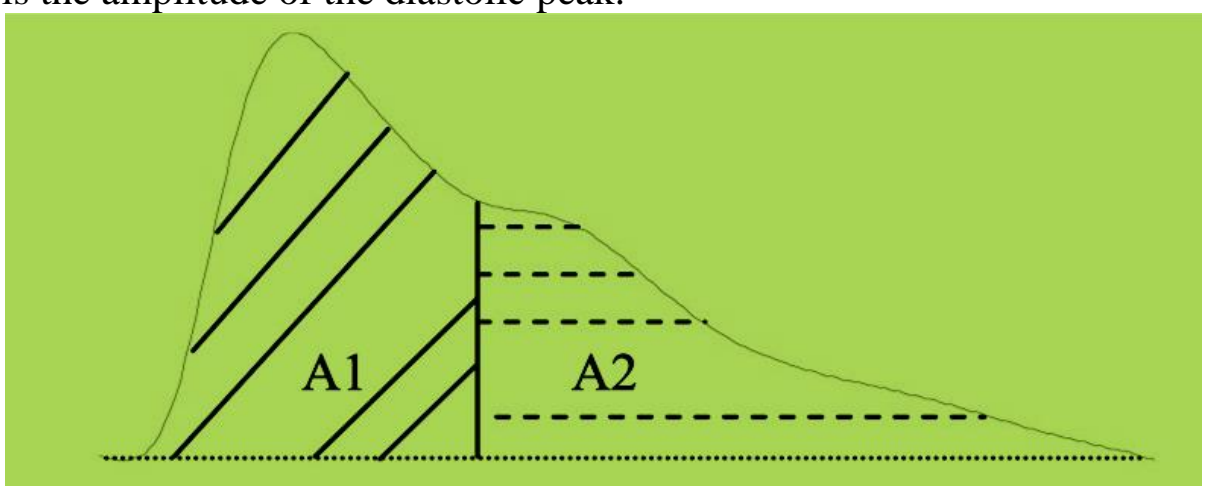

Figure No. 17: Inflection point of a photoplethysmograph

Original fingertip photoplethysmogram. A1 and A2 are the areas under the whole PPG wave separated at the point of inflection. Thus, the inflection point area ration can be calculated as the division of A2 by A1. 


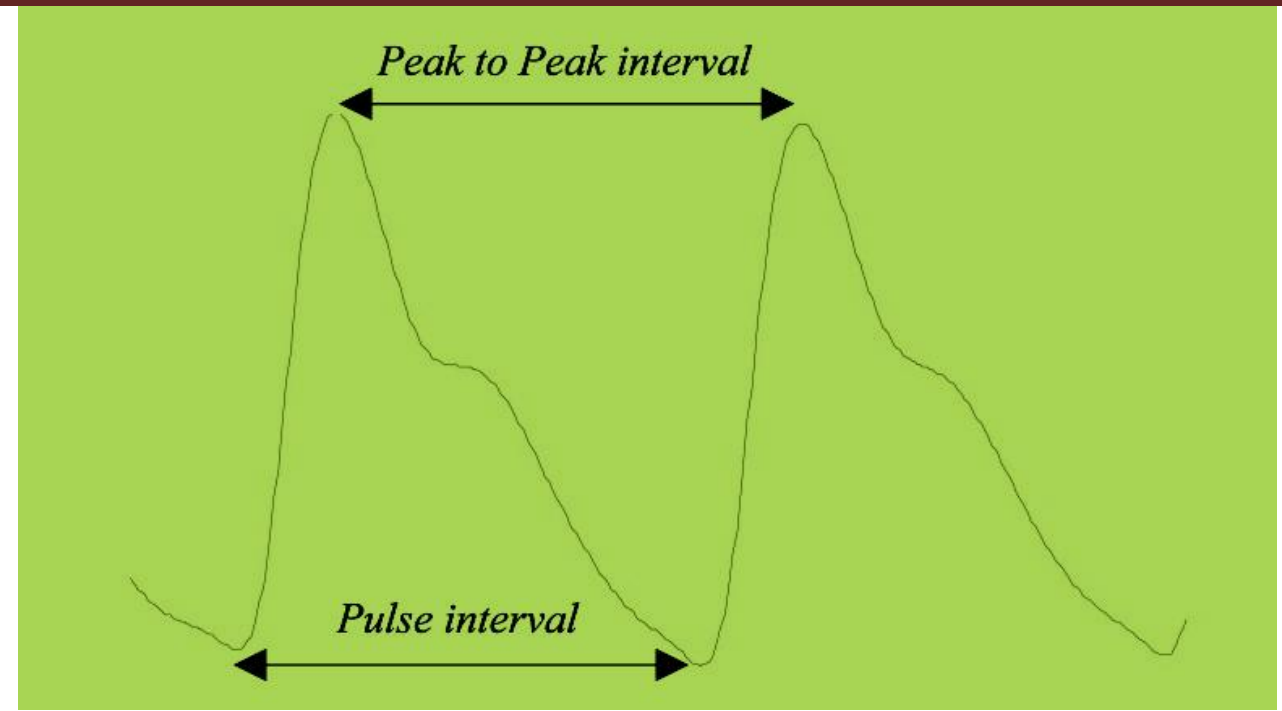

Figure No. 18: Two consecutive PPG waves.

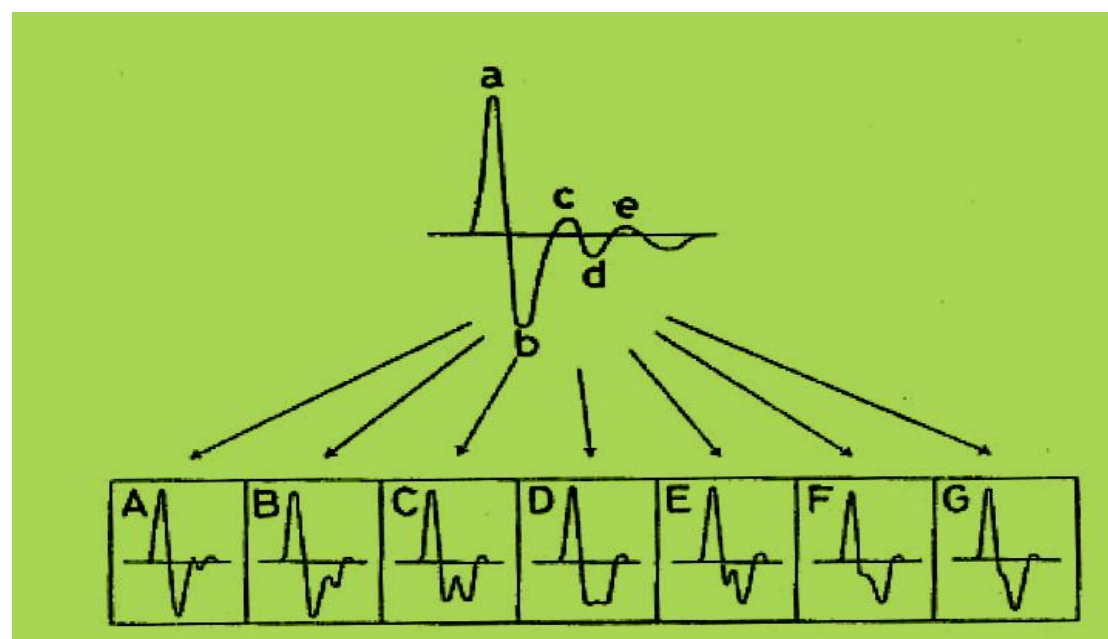

Figure No.19: APG waveforms and types of photoplethysmogram.

There are different types of APG waveforms. The first APG waveform A (far left) refers to good circulation, whereas the amplitude of $b$ wave is lower than $c$ wave. The last APG waveform G (far right) refers to distinctively bad circulation, whereas the amplitude of $\mathrm{c}$ wave is lower than $\mathrm{b}$ wave.

Brian L. Partridge ${ }^{1}$ studied in1987 on use of Pulse Oximetry as a noninvasive indicator of intravascular volume status. The use of pulse oximetery as a noninvasive method to assess intravascular volume status was described. Pulse oximeters providing a continuous display of the pulse waveform offer a new method of estimating relative volume status during positive-pressure ventilation. Like intraarterial pressure tracings, the peaks of the pulse waveform demonstrate increased variation in response to positive-pressure ventilation when a patient becomes hypovolemic. Pulse oximeter waveform tracings were compared with central venous pressure and intraarterial pressure tracings in 12 patients undergoing major operative procedures. A significant correlation $(\mathrm{r}=0.61)$ was seen between pulse waveform variation and systolic pressure variation, which has previously been shown to be a sensitive indicator of hypovolemia. When data from individual patients were analyzed separately, the correlation between pulse waveform variation and systolic pressure variation was as high as 0.88 .

Evans ML, Geddes LA. ${ }^{2}$ studied in 1988 on an assessment of blood vessel vasoactivity using photoplethysmography. The vasoactivity of the vessels of the earlobe, nailbed, finger pad, and nasal septum was investigated in 11 human subjects to determine the suitability of these sites for use in indirect oximetry. The subjects were healthy, nonsmoking volunteers in an age range of 20-32 years. Each site was illuminated with infrared radiation at $880 \mathrm{~nm}$, and the reflected pulsatile signals from the tissue beds were displayed on a multichannel analog recorder. The decrease in pulse amplitude detected at each site in response to a cold- 
pressor test was used as the measure of vasoactivity. Among the sites investigated, the earlobe was the least vasoactive. The nasal septum, nailbed, and finger pad were similar in degrees of vessel reactivity. Hales JR, Stephens FR, Fawcett AA, Daniel K, Sheahan J, Westerman RA, James SB. in $1989^{3}$ studied observations on a new non-invasive monitor of skin blood flow. A 'tissue perfusion monitor' (TPM) to noninvasively provide an index of skin blood flow (SkBF) has been developed; it employs photoelectric plethysmographic principles to measure changes in the net flux of red blood cells in superficial microvasculature. The 'tissue perfusion index' (TPI) varies in proportion to SkBF, provided local haemoglobin concentration does not change significantly. TPI of humans and experimental animals has been shown to indicate reliably, well established phenomena such as decreased SkBF in response to mechanical restriction, cold or Valsalva's manoeuvre, or increased SkBF in response to heat, acetylcholine, sodium nitrite or local nerve blockade. SkBF in sheep was varied between 1 and $156 \mathrm{~mL} / 100 \mathrm{~g}$ per min as measured with radioactive microspheres. Simultaneous measurements were made using the TPM and four laserDoppler instruments. The TPI yielded a correlation coefficient of 0.938 , and when data were expressed as percentage change, the regression line did not differ significantly from the line of identity and the rootmean-square-error was 6.2\%. Data for the laser-Doppler indices of SkBF were, respectively, 0.549-0.786, highly significant deviations in slopes, and 13.6-16.7\%. . Thus, the TPI is a reliable index of changes in SkBF. Compared with some other available instruments, the TPM is more precise; it is also less sensitive to movement artefact, can be completely portable by battery operation, probes can be multiplexed to a single meter and it is likely to be much less expensive than current lasers. Applications include, for example, experimental investigations of SkBF in man and animals, clinical uses such as evaluation of the efficacy of regional nerve blockade or of circulatory restitution after reconstructive surgery, and clinical tests of neurovascular function

Bernardi L, Radaelli A, Solda PL, Coats AJ, Reeder M, Calciati A, Garrard CS, Sleight P studied in $1989^{4}$ autonomic control of skin microvessels: assessment by power spectrum of photoplethysmographic waves.. Although it is well known that the microvessels of the skin constantly undergo spontaneous variations in volume, the significance of these rhythmic changes remains uncertain. In 10 healthy males and in 15 patients in intensive care, they assessed the origin of the autonomic influences on spontaneous fluctuations in the microcirculation of the skin, obtained by an infra-red photoplethysmographic device; they used spectral analysis techniques to compare these fluctuations (which were recorded simultaneously in two sites) with those of blood pressure, in order to test the presence of autonomic control of any synchronous fluctuations in these different measurements from the cardiovascular system. In order to minimize mechanical fluctuations caused by occasional slow breaths, rather than nervously mediated fluctuations in skin blood flow, respiration was controlled at 15 breaths/min $(0.25 \mathrm{~Hz})$. Spontaneous infra-red photoplethysmographic fluctuations were observed in different body areas (left index finger and left ear lobe, right and left index finger), and all were evident at $0.1 \mathrm{~Hz}$, as well as respiration-related components at $0.25 \mathrm{~Hz}$. Active standing increased the power of the $0.1 \mathrm{~Hz}$ fluctuations (sympathetic activity) in both blood pressure (from $62.7+/-7.1$ to $79.2+/-3.7$ normalized units, $\mathrm{P}<0.05$ ) and IRP (finger: from $68.5+/-6.4$ to $86.9+/-3.4$ normalized units, $\mathrm{P}<0.05$; ear: from $59.0+/-5.9$ to $88.1+/-2.0, \mathrm{P}<0.01)$. There was a high (> 0.5 ) coherence between the fluctuations obtained in blood pressure, in IRP signals obtained simultaneously at the finger and at the ear, and in R-R interval. This synchronization between the oscillations in all these signals, which were unrelated to the respiratory frequency or to the pulse rate, suggests a common neural, non-local origin. The phase between IRP and blood pressure was positive in the $0.1 \mathrm{~Hz}$ region $(+1.65+/$ 0.41 radians, i.e. IRP was leading blood pressure, showing that $0.1 \mathrm{~Hz}$ fluctuations were not passively transmitted to the skin microvessels from large arteries) and negative in the $0.25 \mathrm{~Hz}$ region $(-0.74+/-0.19$ radians, $\mathrm{P}<0.01$ compared with phase in the $0.1 \mathrm{~Hz}$ region, i.e. IRP was lagging behind blood pressure, suggesting possible passive transmission to the skin microvessels of blood pressure fluctuations caused by respiration). Fluctuations at lower frequency were observed in all IRP recordings, suggesting a local origin for these. Intra-arterial and IRP fluctuations were compared in the 15 intensive care patients and gave similar results. 4 . The skin microcirculation is thus not only under local control, but also reflects changes in sympathetic activity; the effect of these changes on the skin microcirculation can be easily evaluated by the spectral analysis of the IRP signal obtained simultaneously in multiple areas, in conjunction with the spectra of R-R interval and blood pressure. 
Shamir M, Eidelman LA, Floman Y, Kaplan L, Pizov R. studied in $1999^{5}$ on Pulse oximetry plethysmographic waveform during changes in blood volume.Systolic pressure variation (SPV) and its down component have been shown to be sensitive factors in estimating intravascular volume in patients undergoing mechanical ventilation. In this study, ventilation-induced changes in pulse oximeter plethysmographic waveform were evaluated after removal and after reinfusion of $10 \%$ estimated blood volume. The plethysmographic waveform variation (SPVplet) was measured as the difference between maximal and minimal peaks of waveform during the ventilatory cycle, and expressed as a percentage of the signal amplitude during apnoea. dUp(plet) and dDown(plet) were measured as the distance between the apnoeic plateau and the maximal or minimal plethysmographic systolic waveform, respectively. Intravascular volume was changed by removal of $10 \%$ of estimated blood volume and followed by equal volume replacement with haemaccel. A 10\% decrease in blood volume increased SPVplet from mean 17.0 (SD 11.8)\% to $31.6(28.0) \%(\mathrm{P}=0.005)$ and dDown(plet) from $8.7(5.1) \%$ to $20.5(12.9) \%(\mathrm{P}=0.0005)$ compared with baseline. Changes in plethysmographic waveform correlated with changes in arterial SPV and dDown $(r=0.85 ; P=0.0009)$. In the absence of invasive arterial pressure monitoring, ventilationinduced waveform variability of the plethysmographic signal measured from pulse oximetry is a useful tool in the detection of mild hypovolaemia.

Sinex JE studied in $1999^{6}$ on pulse oximetry: principles and limitations. The pulse oximeter has become an essential tool in the modern practice of emergency medicine. However, despite the reliance placed on the information this monitor offers, the underlying principles and associated limitations of pulse oximetry are poorly understood by medical practitioners. This article reviews the principles of pulse oximetry, with an eye toward recognizing the limitations of this tool. Among these are performance limitations in the settings of carboxyhemoglobinemia, methemoglobinemia, motion artifact, hypotension, vasoconstriction, and anemia. The accuracy of pulse oximetry is discussed in light of these factors, with further discussion of applications for pulse oximetry in emergency medicine, including both oximetric and plethysmographic operation. The pulse oximeter is an invaluable instrument for emergency medicine practice, but as with any test the data it offers must be critically appraised for proper interpretation and utilization.

Kihara S, Yaguchi Y, Watanabe S, Brimacombe J, Taguchi N, Yamasaki Y. studied in 2000 haemodynamic responses to the intubating laryngeal mask and timing of removal.We determined (a) the haemodynamic responses to intubating laryngeal mask (ILM) airway insertion/intubation and removal in anaesthetized patients, and (b) whether the timing of ILM removal influences these responses. One-hundred and twenty patients without cardiovascular disease were studied. ILM airway insertion/intubation was 5 min after induction with propofol $2 \mathrm{mg} \mathrm{kg(-1)}$ and maintenance of anaesthesia with sevoflurane $2 \%$ in oxygen $33 \%$ and nitrous oxide. Patients were randomly assigned for removal of the intubating laryngeal mask airway at 1,3 and $5 \mathrm{~min}$ after successful intubation. Systolic and diastolic arterial pressures and heart rate were recorded preinduction (baseline), before ILM airway insertion/intubation, at 1-min intervals after insertion/intubation, and at 1-min intervals for $5 \mathrm{~min}$ after ILM removal. ILM insertion was successful at the first attempt in all patients, but 46 patients required more than one intubation attempt. Compared with baseline values, there were no increases in systolic or diastolic arterial pressure, but there was an increase in heart rate $1 \mathrm{~min}$ after ILM insertion/intubation $(9 \%, \mathrm{P}<0.001)$ and $1 \mathrm{~min}$ after ILM removal $(8 \%, \mathrm{P}<0.01)$. There was a significant increase in systolic and diastolic pressures and heart rate 1 min after ILM insertion/intubation $(30 \%, 31 \%$ and $15 \%$; all: $\mathrm{P}<0.002)$ compared with before ILM insertion/intubation values and $1 \mathrm{~min}$ after ILM removal $(9 \%, 8 \%$ and $7 \%$; all $\mathrm{P}<0.05)$ compared with 1 min after ILM insertion/intubation values. Removal of the ILM $1 \mathrm{~min}$ after successful intubation resulted in higher arterial pressure compared with removal at $3 \mathrm{~min}$ (systolic arterial pressure 10\% higher for $1 \mathrm{~min}, \mathrm{P}=0.01$ ) and 5 min (systolic arterial pressure 10-23\% higher for $3 \mathrm{~min}, \mathrm{P}<0.01$; diastolic arterial pressure $10-20 \%$ higher for $4 \mathrm{~min}, \mathrm{P}>0.02$ ), but there were no differences in heart rate between groups. Systolic and diastolic arterial pressures were greater if more than one intubation attempt was required. Early removal or multiple intubation attempts did not exceed baseline haemodynamic values. It wasconclude that ILM insertion/intubation and removal in anaesthetized patients produces little or no haemodynamic response, even if multiple intubation attempts are required. The timing of removal exerts a small, but clinically unimportant influence on these responses. ${ }^{7}$ 
Shimoda O, Yoshitake A, Abe E, Koga T studied in 2002 onreflex responses to insertion of the intubating laryngeal mask airway, intubation and removal of the ILMA.They studied 21 patients (ASA 1 or 2) to investigate the skin vasomotor reflex (SVmR) and haemodynamic responses to insertion of an intubating laryngeal mask airway (ILMA), tracheal intubation using the ILMA and removal of the ILMA. Anaesthesia was induced with fentanyl, midazolam, vecuronium and nitrous oxide. A size 4 ILMA was inserted using the standard technique, and a silicone reinforced tracheal tube $(7.5 \mathrm{~mm}$, ID) was passed through it. After confirming successful intubation, the ILMA was removed using the stabilizing rod. The three procedures were performed at approximately one-minute intervals. Insertion of the ILMA, intubation and removal of the ILMA all significantly reduced the skin blood flow on the ring finger in all patients. The mean amplitudes of the SVmR were 0.46 (SD 0.29), $0.54(0.32)$ and 0.68 (0.21) respectively. The magnitude of the SVmR and the haemodynamic changes induced by removal of the ILMA were significantly larger than those accompanying the other two procedures. Use of the ILMA for intubation and removal of the ILMA produces three stimuli and the removal of the ILMA produces the greatest response. ${ }^{8}$

Takahashi S, Tanaka M, Toyooka H.studied in2002 on the efficacy of hemodynamic and T-wave criteria for detecting intravascular injection of epinephrine test dose in propofol-anesthetized adults.A recent study demonstrated $100 \%$ effectiveness of hemodynamic criteria during propofol anesthesia, when a full dose of an epinephrine (15 microg)-containing test dose was injected intravascularly. We designed this doseresponse study to determine minimal effective epinephrine doses and efficacies of hemodynamic and $\mathrm{T}$ wave criteria for detecting intravascular injection of the epinephrine test dose in propofol-anesthetized adults. Eighty healthy adult patients were randomly assigned to one of four groups according to a simulated IV test dose using propofol $(133$ microg center dot $\mathrm{kg}(-1)$ center dot $\min (-1))$ and nitrous oxide $(\mathrm{FIO}(2)=$ $0.33)$ anesthesia after endotracheal intubation $(\mathrm{n}=20$ each). The Saline group received $3 \mathrm{~mL}$ of normal saline IV; the Epinephrine-15 group received $3 \mathrm{~mL}$ of $1.5 \%$ lidocaine containing 15 microg epinephrine; and the Epinephrine-10 and -5 groups received 2 and $1 \mathrm{~mL}$ of the test dose of the identical components, respectively. Heart rate (HR), systolic blood pressure (SBP), and lead II of the electrocardiogram were recorded continuously for $5 \mathrm{~min}$ after the IV injection of the study drug via a peripheral vein. Sensitivities and specificities of $100 \%$ were obtained based on the modified HR (positive if greater-than-or-equal to 10 bpm increase) and the T-wave (positive if greater-than-or-equal 25\% in amplitude) criteria if greater-thanor-equal 5 microg of epinephrine was injected IV. Based on the SBP criterion (positive if greater-than-orequal $15 \mathrm{~mm} \mathrm{Hg}$ increase), however, $100 \%$ sensitivity and specificity were associated only with greaterthan-or-equal 10 microg of epinephrine doses. These results suggest that the minimal effective epinephrine doses for detecting unintentional intravascular injection are 5 microg based on the HR and T-wave criteria, and 10 microg based on the SBP criterion in adult patients anesthetized with propofol and nitrous oxide. Accidental migration of an epidural catheter into a blood vessel is often detected by hemodynamic changes after injecting an epidural test dose containing epinephrine. Our results suggest that 5 microg of epinephrine is not adequate to reliably produce hemodynamic and T-wave alterations in adult patients during propofol anesthesia. ${ }^{9}$

Yash Pole studied Evolution of the pulse oximeterPulse oximetry in 2002has revolutionized the concept of clinical monitoring. No other electronic monitoring device has found widespread use in the operating room more quickly than has the pulse oximeter. Oximetry is the spectrophotometric determination of the hemoglobin-oxygen saturation. The pulse oximeter is completely noninvasive and gives continuous realtime estimates of arterial-oxygen saturation. As of 1988, the AAGB\&I has recognized it as their standard for intraoperative monitoring. As of January 1, 1990, it became an ASA standard for intraoperative monitoring. This standard was adopted with the expectation that use of the instrument would improve patient safety. But who invented this modern wonder gadget? Perhaps the names of Dr. Takuo Aoyagi, the inventor, and Prof. Susumu Nakajima, the pioneer, and William New, Mark Yelderman and Jack Lloyd, who brought the pulse oximeter into the operating rooms, ought to be remembered, as they were the ones who recognized the potential importance of pulse oximetry and developed interest among anesthesiologists. However, it was Prof. Karl Matthes, a professor of physiology from Vienna, who is regarded as the Father of Oximetry for the dual achievement of constructing the first continuously reading oximeter in 1935 and of stating its principle. This paper presents the historical evolution of the pulse oximeter starting from Isaac Newton in $1666 .{ }^{10}$ 
Robert F. Tamburro, John C. Ring, Kimberly Womback studied in 2002 onDetection of Pulsus Paradoxus Associated With Large Pericardial Effusions in Pediatric Patients by Analysis of the Pulse-Oximetry WaveformObjective. To determine whether analysis of the pulse-oximetry waveform can be useful in detecting the pulsus paradoxus associated with large pericardial effusions in pediatric patients.Methods. A retrospective review of charts of 8 pediatric patients (age range: 5-19 years) who had echocardiographic evidence of large pericardial effusion, subsequently underwent pericardiocentesis, and had pulse-oximetry waveform tracings obtained before and after pericardiocentesis within an 18-month period was conducted in 2 tertiary-care pediatric intensive care units. They analyzed the pulse-oximetry waveform tracings for the presence of a pulsus paradoxus. Other abstracted data included clinical evidence of tamponade, echocardiographic findings, and the volume of pericardial fluid aspirated.Results. Before pericardiocentesis, a decrease in the highest value of the upper plethysmographic peak of the pulse-oximetry waveform was observed during inspiration in each patient. Echocardiographic evidence of large pericardial effusion with compromised cardiac filling was also present in each patient. Only 6 of these patients had clinical evidence of cardiac tamponade at that time, 4 with a documented pulsus paradoxus using standard methods of blood pressure analysis. After pericardiocentesis, the inspiratory fall in the highest value of the upper plethysmographic peak of the pulse-oximetry waveform lessened in every patient. Echocardiography documented a decrease in the size of the effusion and resolution of the compromised cardiac filling in every patient.Conclusions. Analysis of pulse-oximetry waveforms may be a widely available, easily interpretable, and reliable method of detecting the pulsus paradoxus associated with large pericardial effusions in pediatric patients. ${ }^{11}$

Barak M, Ziser A, Greenberg A, Lischinsky S, Rosenberg B. studied in2003 on hemodynamic and catecholamine response to tracheal intubation: direct laryngoscopy compared with fiberoptic intubation.To compare the stress response following tracheal intubation using direct laryngoscopy to that using fiberoptic bronchoscopy technique.It was aRandomized, prospective study.51 ASA physical status I and II patients who were scheduled for anPatients were randomly assigned to receive either direct laryngoscopy or fiberoptic orotracheal intubation, as part of general anesthesia. A uniform protocol of anesthetic medications was used.Blood pressure and heart rate were measured before induction, before endotracheal intubation, and 1, 2, 3, and 5 minutes afterwards. Catecholamine (epinephrine and norepinephrine) blood samples were drawn before the induction, and 1 and 5 minutes after intubation.Duration of intubation was shorter in the direct laryngoscopy group (16.9 (16.9 +/- $7.0 \mathrm{sec}$, range 8 to 40) compared with the fiberoptic intubation group $(55.0+/-22.5 \mathrm{sec}$, range 29 to 120$), \mathrm{p}<0.0,001$. In both groups, blood pressure and heart rate were significantly increased at 1,2 , and 3 minutes after intubation, but there was no significant difference between the two study groups. Catecholamine levels did not increase after intubation and did not correlate with the hemodynamic changes. The use of either direct laryngoscopy or fiberoptic bronchoscopy produces a comparable stress response to tracheal intubation. Catecholamine levels do not correlate with the hemodynamic changes. ${ }^{12}$

Koppel RI, Druschel CM, Carter T, Goldberg BE, Mehta PN, Talwar R, Bierman FZ. studied in2003 oneffectiveness of pulse oximetry screening for congenital heart disease in asymptomatic newborns.to determine the sensitivity, specificity, predictive value, and accuracy of a program of pulse oximetry screening of asymptomatic newborns for critical congenital cardiovascular malformation (CCVM).Pulse oximetry was performed on asymptomatic newborns in the well-infant nurseries of 2 hospitals. Cardiac ultrasound was performed on infants with positive screens (saturation <or $=95 \%$ at $>24$ hours). Data regarding true and false positives as well as negatives were collected and analyzed.Oximetry was performed on 11281 asymptomatic newborns, and 3 cases of CCVM were detected (total anomalous pulmonary venous return $\mathrm{x} 2$, truncus arteriosus). During the study interval, there were 9 live births of infants with CCVM from a group of 15 fetuses with CCVM detected by fetal echocardiography. Six infants with CCVM were symptomatic before screening. There was 1 false-positive screen. Two infants with negative screens were readmitted (coarctation, hypoplastic left pulmonary artery with aorto-pulmonary collaterals). Other cardiac diagnoses in the database search were nonurgent, including cases of patent foramen ovale, peripheral pulmonic stenosis, and ventricular septal defect. The prevalence of critical CCVM among all live births was 1 in 564 and among the screened population was 1 in 2256 (sensitivity: 60\%; specificity: 99.95\%; positive predictive value: $75 \%$; negative predictive value: $99.98 \%$; accuracy: $99.97 \%$ ).This screening test is simple, 
noninvasive, and inexpensive and can be administered in conjunction with state-mandated screening. The false-negative screen patients had lesions not amenable to detection by oximetry. The sensitivity, specificity, and predictive value in this population are satisfactory, indicating that screening should be applied to larger populations, particularly where lower rates of fetal detection result in increased CCVM prevalence in asymptomatic newborns. ${ }^{13}$

Hager H., Church S., Mandadi G., Pulley D., Kurz A. in the proceedings of the 2004 Annual Meeting of the American Society of Anesthesiologists:Perfusion Index Measured By a Pulse Oximeter Indicates Pain Stimuli in anesthetized Volunteers.Under physiologic conditions peripheral perfusion is regulated by the autonomic nerve system. During anesthesia this system is impaired due to the vasodilative effect of anesthetic drugs. This effect leads to redistribution of temperature and temperature loss and can effectively be monitored by the forearm fingertip temperature gradient. A new method of measuring peripheral perfusion is the perfusion index displayed on a pulse oximeter. The perfusion index is measured by the pulse oximeter for internal signal quality evaluation and is the relation between pulsatile and constant absorbed light in the finger. Major determinant of the perfusion index is vasoconstriction or vasodilation of peripheral vessels due to temperature, volume status and endtidal inhaled anesthetics like Sevoflurane.(1) Also pain is known to induce vasoconstriction, but it is unknown if this effects peripheral perfusion under the vasodilated condition in normothermic anesthetized subjects. We obtained informed consent after IRB approval from 2 healthy volunteers aged 28 and 33. The volunteers were monitored with ECG, NIBP and a Masimo®Radical SET pulse oximeter (Irvine, CA, USA) displaying the perfusion index. Anesthesia was induced using Propanol $2 \mathrm{mg} / \mathrm{kg}$ and maintained with Sevoflurane as a single agent anesthesia. We assigned the volunteers to 4 different concentrations of Sevoflurane $(1 \%, 1,5 \%, 2 \%$ and 2,5\%) in random order. In each condition we applied a standardized painful (electrical) stimulus via two 25-g needles which were inserted intradermally into the lower portion of each anterior thigh. A bilateral 65-70 milliampere, 100-Hertz tetanic electrical current, maintained for 10 seconds, provided the noxious stimulus.As expected the painful stimulus caused significant increase in heart rate from $62.5 \pm 9.5$ to $80.38 \pm 13.18(\mathrm{p}=0.01)$ and a significant increase in mean arterial pressure from $70.75 \pm 9.44$ to $92 \pm 15.11(\mathrm{p}=0.005)$. The average baseline perfusion index before each stimulus was $11.07 \pm 1.19$. Painful stimulus caused a significant decline of the perfusion index $5.42 \pm 2.39$ ( $\mathrm{p}<0.001)$. There was a weak correlation between endtidal sevoflorane concentration and perfusion index. ( $\mathrm{r} 2=0.343$ ) There was also a weak correlation between end-tidal sevoflurane concentration and the decline of the perfusion index during painful stimulus $(\mathrm{r} 2=-0.4)$. The perfusion index is able to independently indicate a pain stimulus in anesthetized volunteers in different concentrations of Sevoflurane. Thus it may be of clinical value to assess pain. ${ }^{14}$

Kakazu CZ, Chen BJ, Kwan WF. studied in2005Masimo SET Technology Using PerfusionIndex Is a Sensitive Indicator for EpiduralOnsetPerfusion Index (PI) reflects the degree of pulsatile blood flow at the monitoring site. Various factors including vascular tone can influence the PI values. The authors have previously demonstrated that increases in PI values are a rapid and reliable indication of a functioning epidural catheter. The authors conducted this study to determine the sensitivity of the PI value to small test doses of epidural anesthetic. The benefit of this would be that the functionality and placement of the epidural catheter could be determined prior to the infusion of the full anesthetic dose.A total of 16 adult women undergoing labor had a Masimo Radical attached to the toe prior to epidural catheter placement. Baseline values for blood pressure, heart rate and PI were noted and recorded every minute thereafter. Following this, an epidural catheter was placed and a small test dose of local anesthetic was administered. Five minutes later the epidural catheter was infused with incremental doses of $0.25 \%$ bupivicaine.Paired t-test comparing baseline PI vs 5 min PI, baseline PI vs 20 min PI and 5 min PI vs 20 min PI shown below, demonstrate significant changes in PI over time following infusion of anesthetic test dose. The box plot represents the distribution (with the lowest bar representing the 10th percentile and the upper most bar representing the 90th percentile of the data) of the PI values at each of the time periods; at baseline, 5 minutes, and 20 minutes. $^{15}$

In article STRESS RESPONSE TO TRAUMA AND SURGERY it has been stated that insulin concentration may decrease after induction of anesthesia and during surgery there is a failure o insulin secretion to match the catabolic,hyperglycemic response.this may be partly by alfa adrenergic inhibition of $b$ cell secretion .In addition there is afaliure of the usual cellular response to insulin , the so called "insulin 
resistance"promotes hepatic glycogenolysis.It also increases gluconeogenesis from aminoacid in the liver and has lipolytic activity.Although plasma glucagon concentration increases transiently after major sugery ,thus does not make a major contribution to hyperglycemic response during surgery.Cortisol secretion from the adrenal cortex increases rapidily following the start of surgery, as a result of stimulation by ACTH. From base line value of around 400nmol per litre, cortisol concentration increase to a maximum at about 4-6 hours and may reach $>1500 \mathrm{nmol}$ perlitre depending on the severity of traumait promote protein breakdown and gluconeogenesis .glucose use by cells are inhibited so that blood glucose levels remain high.Blood glucose levels concentration increases after surgery begins.Cortisol and catecholamnnes facilitates glucose production as a result of increase hepatic glycogenolysis and gluconeogenesis. In addition peripheral use of glucose decreased.blood glucose level are related to intensity of surgical injury; the changes follow closely the increase in catecholamines.In cardiac surgery, blood glucose level can increase upto 10-12 mmol per litre and remain elevated for $>24$ hour after surgery.The changes are less marked with minor surgery.The usual mechanisms that maintain glucose hemostasis are ineffective in the perioperative period.hyperglycemia persist because catabolic hormones promote glucose production and there is a relative lack of insulin together with peripheral insuline resistanceStress response and surgical outcomes There has been a great deal of interest in the modification of the stress response with respect to the potential beneficial effect on surgical outcome .The extent to which the responses are modified depends upon the choice of the analgesic technique used. Inhibition of stress responses is greatest with neural blockade with local anesthetics. Therefore attention has focused largely on the effects of regional anesthetics and analgesic regimens, particularly epidural blockade with local anesthetic agents. Individual studies has shown that provision of analgesia using neural blockade leads to improvement in physiological variables in specific organ system. single investigation often cannot show benefits in morbidity and mortality because the incidence of serious complications after analgesia has beneficial effect on surgical outcome. ${ }^{16}$

Lima A, Bakker J.studied in 2005 Noninvasive monitoring of peripheral perfusionBackground: Perfusion index (PI) is a non-invasive numerical value of peripheral perfusion obtained from a pulse oximeter. In this study, we evaluated the efficacy of PI for detecting haemodynamic stress responses to insertion of i-gel, laryngeal mask airway (LMA) and endotracheal tube and compare, its reliability with the conventional haemodynamic criteria in adults during general anaesthesia. Methods: Sixty patients scheduled for elective general surgery under general anaesthesia were randomised to three groups. (i-gel, LMA and ET groups ( $n=20$ /group). Heart rate (HR) (positive if $\geq 10 \mathrm{bpm}$ ), systolic blood pressure (SBP), diastolic blood pressure (DBP) (positive if $\geq 15 \mathrm{~mm} \mathrm{Hg}$ ) and PI (positive if $\leq 10 \%$ ) were monitored for $5 \mathrm{~min}$ after insertion. Main outcome measures: SBP, DBP, HR and PI were measured before induction of anaesthesia and before and after insertion of the airway device. Results: Insertion of airway devices produced significant increases in HR, SBP and DBP in LMA and ET groups. Moreover, PI was decreased significantly by 40\%, 100\% and $100 \%$ in the three groups. Using the PI criterion, the sensitivity was 100\% (CI 82.4-100.0\%). Regarding the SBP and DBP criterions, the sensitivity was 44.4\% (CI 24.6-66.3\%), 55.6\% (CI 33.7-75.4\%) respectively. Also, significant change in the mean PI over time (from pre-insertion value to the $1 \mathrm{st} \mathrm{min,} 3 \mathrm{rd}$ min, until the 4 th min after insertion without regard the device type), $(\mathrm{P}<0.001)$. Conclusion: PI is a reliable and easier alternative to conventional haemodynamic criteria for detection of stress response to insertion of i-gel, LMA and ET during propofol fentanyl isoflurane anaesthesia in adult patients. ${ }^{17}$

Takenaka K, Ogawa E, Wada H, Hirata T. studied in 2006 Systemic inflammatory response syndrome and surgical stress in thoracic surgery.To evaluate the clinical usefulness of postoperative systemic inflammatory response syndrome (SIRS) as an index of surgical stress in patients undergoing thoracic surgery.Forty-five consecutive patients who underwent thoracic surgery with thoracotomy were enrolled. The SIRS criteria were examined daily during the first 7 postoperative days. The serum interleukin-6 (IL-6) level, operation time, intraoperative blood loss, amount of thoracic drainage, and C-reactive protein levels were also measured.Sixteen cases were categorized into the SIRS group, whereas 29 cases were categorized into the non-SIRS group. Among the patients who underwent thoracic surgery, the physiological responses of the patients to the surgery, such as serum IL-6 levels and C-reactive protein levels, were significantly higher in the SIRS group than in the non-SIRS group $(\mathrm{P}=.002$ and .024 , respectively). The serum IL-6 level on the first postoperative day was an independent factor associated with SIRS (95\% CI 1.002-1.041; P $=.030$ ). Furthermore, there was a correlation between the number of SIRS days and the duration of the postoperative 
hospital stay $(\mathrm{r}=0.379, \mathrm{P}=.012)$. Our results demonstrated that SIRS reflected the degree of surgical stress, especially thoracotomic procedures, through the IL-6 levels, and affected the postoperative hospital stay. Systemic inflammatory response syndrome can be useful for the postoperative management of patients undergoing thoracic surgery. ${ }^{18}$

Galvin EM1, Niehof S, Verbrugge SJ, Maissan I, Jahn A, Klein J, van Bommel J. studied on peripheral flow index is a reliable and early indicator of regional block success.in $2006 \mathrm{We}$ investigated the usefulness of peripheral flow index (PFI) measurement using a standard pulse oximetry digit probe for early prediction of successful regional blocks. Sixty-six patients scheduled for limb surgery underwent either axillary or sciatic block using a nerve stimulator technique with mepivacaine $1.5 \%$. PFI, which is the ratio of the pulsatile versus the nonpulsatile component of the pulse oximetry signal, was recorded from 10 min before block insertion until $30 \mathrm{~min}$ afterwards. PFI recordings of the unblocked limb were similarly recorded. Pinprick and cold sensation were assessed at 5-min intervals until 30 min after blockade. An increase in PFI by a factor of 1.55 at $10 \mathrm{~min}$ after axillary block placement $(\mathrm{P}=0.006)$, and $12 \mathrm{~min}$ after sciatic block placement $(\mathrm{P}=0.001)$ was required to predict a successful block. The sensitivity and specificity of PFI was $100 \%$ for predicting axillary block outcomes at this time. Positive predictive value was $95 \%$ and negative predictive value was $93 \%$. For sciatic blocks, sensitivity and specificity were $90 \%$ and $100 \%$, respectively. The calculated positive predictive value at time 12 min for sciatic blocks was $94 \%$ and negative predictive value was $92 \%$. At 15 min after block placement, cold and pinprick sensations had the same calculated values for sensitivity and specificity at $71 \%$ and $100 \%$, respectively, for axillary blocks. For sciatic blocks, cold sensation had a sensitivity of $77 \%$ and a specificity of $100 \%$, whereas pinprick had a sensitivity of just $20 \%$ with a specificity of $100 \%$. We conclude that PFI provides a simple, early, and objective assessment of the success and failure of nerve blocks. ${ }^{19}$

Feissel M1, Teboul JL, Merlani P, Badie J, Faller JP, Bendjelid K. studied in 2007 on pethysmographic dynamic indices predict fluid responsiveness in septic ventilated patients.In septic patients, reliable noninvasive predictors of fluid responsiveness are needed. We hypothesised that the respiratory changes in the amplitude of the plethysmographic pulse wave (DeltaP(PLET)) would allow the prediction of changes in cardiac index following volume administration in mechanically ventilated septic patients.Prospective clinical investigation.setting:An 11-bed hospital medical intensive care unit.Twenty-three deeply sedated septic patients mechanically ventilated with tidal volume $>$ or $=8 \mathrm{ml} / \mathrm{kg}$ and equipped with an arterial catheter and a pulse oximetry plethysmographic sensor.Respiratory changes in pulse pressure (DeltaPP), DeltaP(PLET) and cardiac index (transthoracic Doppler echocardiography) were determined before and after volume infusion of colloids $(8 \mathrm{ml} / \mathrm{kg}$ ). Twenty-eight volume challenges were performed in 23 patients. Before volume expansion, DeltaPP correlated with DeltaP(PLET) $(\mathrm{r} 2=0.71, \mathrm{p}<0.001)$. Changes in cardiac index after volume expansion significantly $(\mathrm{p}<0.001)$ correlated with baseline DeltaPP $(\mathrm{r} 2=0.76)$ and DeltaP(PLET) $(\mathrm{r} 2=0.50)$. The patients were defined as responders to fluid challenge when cardiac index increased by at least $15 \%$ after the fluid challenge. Such an event occurred 18 times. Before volume challenge, a DeltaPP value of $12 \%$ and a DeltaP(PLET) value of $14 \%$ allowed discrimination between responders and non-responders with sensitivity of $100 \%$ and $94 \%$ respectively and specificity of $70 \%$ and $80 \%$ respectively. Comparison of areas under the receiver operator characteristic curves showed that DeltaPP and DeltaP(PLET) predicted similarly fluid responsiveness. The present study found DeltaP(PLET) to be as accurate as DeltaPP for predicting fluid responsiveness in mechanically ventilated septic patients. ${ }^{20}$ Awad AA1, Haddadin AS, Tantawy H, Badr TM, Stout RG, Silverman DG, Shelley KH. studied in 2007 the relationship between the photoplethysmographic waveform and systemic vascular resistance. The objective of this study was to determine the relationship between systemic vascular resistance (SVR), finger \& ear photoplethysmographic measurements in 14 adult patients undergoing coronary artery bypass grafting (CABG)Patients were monitored with photoplethysmographs of the finger and ear and continuous cardiac output (QT) via thermodilution catheter. The relationship between SVR, finger plethysmographic amplitude, width and ear plethysmographic amplitude, width was assessed with linear regression.The finger plethysmographic amplitude had a low correlation $r$ value $=-0.15$, while finger plethysmographic width had a better correlation $r$ value $=0.56$. The correlation between SVR and ear plethysmographic amplitude and width were -0.24 and 0.62 respectively. Using receiver operating characteristic analysis the ear plethysmographic width had both better sensitivity and specificity than the finger plethysmographic width in 
identifying high and low SVR. Using a multiple regression analysis, SVR was estimated from the pulse oximeter waveforms: SVR calculated $=27.27+(3978.53 \times$ Ear pulse oximeter width $)-(8.91 \times$ Ear pulse oximeter area $)+(1986.3 \times$ Finger pulse oximeter width). Bland-Altman analysis was used the bias was 29.8 dynes s cm(-5), standard deviation was 587.3, upper and lower limit of agreement were 1204.45, and 1144.8 dynes $\mathrm{s} \mathrm{cm}(-5)$ respectively. The data indicate that pulse width of finger and ear plethysmographic tracing are more sensitive to changes in SVR than the other indices. An appreciation of changes in pulse width may provide valuable evidence with respect to changes in peripheral vascular tone. ${ }^{21}$

Allen J. studied in 2007 on photoplethysmography and its application in clinical physiological measurement.Photoplethysmography (PPG) is a simple and low-cost optical technique that can be used to detect blood volume changes in the microvascular bed of tissue. It is often used non-invasively to make measurements at the skin surface. The PPG waveform comprises a pulsatile ('AC') physiological waveform attributed to cardiac synchronous changes in the blood volume with each heart beat, and is superimposed on a slowly varying ('DC') baseline with various lower frequency components attributed to respiration, sympathetic nervous system activity and thermoregulation. Although the origins of the components of the PPG signal are not fully understood, it is generally accepted that they can provide valuable information about the cardiovascular system. There has been a resurgence of interest in the technique in recent years, driven by the demand for low cost, simple and portable technology for the primary care and community based clinical settings, the wide availability of low cost and small semiconductor components, and the advancement of computer-based pulse wave analysis techniques. The PPG technology has been used in a wide range of commercially available medical devices for measuring oxygen saturation, blood pressure and cardiac output, assessing autonomic function and also detecting peripheral vascular disease. The introductory sections of the topical review describe the basic principle of operation and interaction of light with tissue, early and recent history of PPG, instrumentation, measurement protocol, and pulse wave analysis. The review then focuses on the applications of PPG in clinical physiological measurements, including clinical physiological monitoring, vascular assessment and autonomic function. ${ }^{22}$

Granelli Ad, Ostman-Smith I. studied in 2007on noninvasive peripheral perfusion index as a possible tool for screening for critical left heart obstruction.Peripheral perfusion index (PPI) has been suggested as a possible method to detect illness causing circulatory embarrassment. We aimed to establish the normal range of this index in healthy newborns, and compare it with newborns with duct-dependent systemic circulation. it was a case-control study. Their study population comprised 10,000 prospectively recruited newborns from Västra Götaland, Sweden.A total of 10,000 normal newborns and 9 infants with duct-dependent systemic circulation (left heart obstructive disease [LHOD] group) participated in the study.They conducted single pre- and postductal measurements of PPI with a new generation pulse oximeter (Masimo Radical SET) before discharge from hospital.PPI values between 1 and $120 \mathrm{~h}$ of age show an asymmetrical, non-normal distribution with median PPI value of 1.70 and interquartile range of 1.18-2.50. The 5th percentile $=0.70$ and 95th percentile $=4.50$. All infants in the LHOD group had either pre- or postductal PPI below the interquartile range, and 5 of $9(56 \%)$ were below the 5 th percentile cut-off of 0.70 (p $<0.0001$, Fisher's exact test). A PPI value $<0.70$ gave an odds ratio for LHOD of 23.75 (95\% CI 6.36-88.74).PPI values lower than 0.70 may indicate illness and a value $<0.50$ (1st percentile) indicates definite underperfusion. PPI values might be a useful additional tool for early detection of LHOD. ${ }^{23}$

Takuo Aoyagi: discovery of pulse oximetry.In the 1930s and 1940s, photo cells permitted German, English, and American physiologists to construct ear oximeters with red and infrared light, requiring calibration. In 1940 Squire recognized that changes of red and infrared light transmission caused by pneumatic tissue compression permitted saturation to be computed. In 1949 Wood used this idea to compute absolute saturation continuously from the ratios of optical density changes with pressure in an ear oximeter. In 1972 Takuo Aoyagi, an electrical engineer at Nihon Kohden company in Tokyo, was interested in measuring cardiac output noninvasively by the dye dilution method using a commercially available ear oximeter. He balanced the red and infrared signals to cancel the pulse noise which prevented measuring the dye washout accurately. He discovered that changes of oxygen saturation voided his pulse cancellation. He then realized that these pulsatile changes could be used to compute saturation from the ratio of ratios of pulse changes in the red and infrared. His ideas, equations and instrument were adapted, improved and successfully marketed 
by Minolta about 1978, stimulating other firms to further improve and market pulse oximeters worldwide in the mid 1980s. Dr. Aoyagi and associates provided a detailed history for this paper. ${ }^{24}$

Shelley KH. studied on Photoplethysmography: beyond the calculation of arterial oxygen saturation and heart rate in2007.In this article, I examine the source of the photoplethysmograph (PPG), as well as methods of investigation, with an emphasize on amplitude, rhythm, and pulse analysis. The PPG waveform was first described in the 1930s. Although considered an interesting ancillary monitor, the "pulse waveform" never underwent intensive investigation. Its importance in clinical medicine was greatly increased with the introduction of the pulse oximeter into routine clinical care in the 1980s. Its waveform is now commonly displayed in the clinical setting. Active research efforts are beginning to demonstrate a utility beyond oxygen saturation and heart rate determination. Future trends are being heavily influenced by modern digital signal processing, which is allowing a re-examination of this ubiquitous waveform. Key to unlocking the potential of this waveform is an unfettered access to the raw signal, combined with standardization of its presentation, and methods of analysis. In the long run, we need to learn how to consistently quantify the characteristics of the PPG in such a way as to allow the results from research efforts be translated into clinically useful devices.Impact of withdrawal of $450 \mathrm{ml}$ of blood on respiration-induced oscillations of the ear plethysmographic waveform. ${ }^{25}$

Gesquiere MJ, Awad AA, Silverman DG, Stout RG, Jablonka DH, Silverman TJ, Shelley KH. studie in 2007 onimpact of withdrawal of $450 \mathrm{ml}$ of blood on respiration-induced oscillations of the ear plethysmographic waveform.It has been widely appreciated that ventilation-induced variations in systolic blood pressure during mechanical ventilation correlate with changes in intravascular volume. The present study assessed whether alterations in volume status likewise can be detected with noninvasive monitoring (ear plethysmograph) in non-intubated subjects (awake volunteers).Eight healthy adults were monitored with EKG, noninvasive blood pressure, an unfiltered ear plethysmograph, and a respiratory force transduction belt before (pre) and after (post) withdrawal of $450 \mathrm{ml}$ of blood from an antecubital vein. Spectral-domain analysis was used to determine the peak ventilatory frequency and the power of the associated variation in the ear plethysmographic tracing; Interphase differences in the respiration-induced plethysmographic variations were assessed by Wilcoxon signed rank test. In addition, the changes in the ear plethysmographic tracing were compared to changes in heart rate and blood pressure. There was a significant increase in respiratory-associated oscillations at the respiratory frequency between the pre and post phases $(\mathrm{p}=0.012)$. These changes were detected despite lack of changes in heart rate or blood pressure.Respiration-induced changes of the ear plethysmographic waveform during spontaneous ventilation increase significantly as a consequence of withdrawal of approximately one unit of blood in healthy volunteers. ${ }^{26}$

Raamat R, Jagomägi K, Talts J. studied in2007 on calibrated photoplethysmographic estimation of digital pulse volume and arterial compliance.Digital pulse volume and arterial compliance have been estimated by using the finger arterial pressure and volume waveforms when performing measurements at rest and during local cold exposure in 17 volunteers. The amplitude-based algorithm determines compliance as a ratio of finger volume and pressure pulses. Cooling was conducted by immersion of the contra-lateral arm in water at 11 degrees $\mathrm{C}$ for $2 \mathrm{~min}$. Continuous non-invasive finger blood pressure was measured by the Finapres monitor, while the finger volume pulses were recorded by the UT9201 physiograph, which provides a photoplethysmographic registration of volume changes in a large range of transmural pressures $(\mathrm{P}(\operatorname{transm}))$. The photoplethysmographic signal was calibrated by applying a graded modulation to the cuff. The study demonstrated that contra-lateral arm cooling induced a statistically significant $(\mathrm{P}<0.001)$ reduction in the digital arterial compliance at $\mathrm{P}($ transm) approximately 0 as well as at $\mathrm{P}($ transm $)=50 \mathrm{mmHg}$. The median compliance diminished during cooling from $0.67 \mathrm{~mm}(3) \mathrm{mmHg}(-1)$ to the level of $55 \%$ at $\mathrm{P}(\operatorname{transm})$ approximately 0 , and from $0.14 \mathrm{~mm}(3) \mathrm{mmHg}(-1)$ to the level of $42 \%$ at $\mathrm{P}($ transm $)=50 \mathrm{mmHg}$ respectively. The observed changes were mostly due to the reduction in the pulse volume. Digital arterial pressure and volume waveforms recorded by the Finapres monitor combined with a calibrated photoplethysmograph can be used in estimating the absolute values of the dynamic compliance of finger arteries. A quantitative estimation instead of the non-calibrated recording of the digital pulse volume allows shortening the variability and widening the scope of finger arterial compliance measurements. ${ }^{27}$

Frey B, Waldvogel K, Balmer C studied in 2008 on clinical applications of photoplethysmography in paediatric intensive careThe photoplethysmographic wave is displayed by most pulse oximeters. It may be 
used as a non-invasive alternative to invasive arterial blood pressure trace analysis for continuous haemodynamic monitoring in selected situations.Four cardiac patients treated in a tertiary neonatalpaediatric intensive care unit.Simultaneous monitoring of the photoplethysmographic wave, ECG, and invasive blood pressure.Photoplethysmography allows for monitoring pulse rate in patients with (possible) heart rate/pulse rate dissociation (pacemaker dependency, pulsatile ventricular assist device); monitoring sudden changes in heart beat volume, which are unrelated to respiration (pulseless electrical activity, pulsus alternans); and monitoring respiratory-dependent fluctuations of the plethysmographic wave (heart failure, hypovolaemia, asthma, upper airway obstruction, pericardial effusion). Deterioration, slowly evolving over time, may be detected by this method. ${ }^{28}$

Mowafi HA, Arab SA, Ismail SA, Al-Ghamdi AA, Al-Metwalli RR studied in 2008 on plethysmographic pulse wave amplitude is an effective indicator for intravascular injection of epinephrine-containing epidural test dose in sevoflurane-anesthetized pediatric patientsPlethysmographic pulse wave amplitude (PPWA) was effective in detecting intravascular injection of epidural test dose with $100 \%$ sensitivity and specificity in adults. We evaluated the efficacy of PPWA in detecting intravascular injection of a simulated epidural test dose during sevoflurane anesthesia in pediatric patients.Eighty infants and children were randomized to receive either 0.5 minimal alveolar concentration (MAC) or 1 MAC sevoflurane and nitrous oxide in oxygen. Patients in each anesthesia group were further randomized to receive either $0.1 \mathrm{~mL} / \mathrm{kg}$ of $1 \%$ lidocaine with 1:200,000 epinephrine $(0.5 \mathrm{mug} / \mathrm{kg}$ of epinephrine) IV to simulate the intravascular injection of epidural test dose or saline. Heart rate (HR), systolic blood pressure (SBP), and PPWA were monitored for $5 \mathrm{~min}$ after injection. A positive test response was defined as HR increase > or $=10 \mathrm{bpm}, \mathrm{SBP}$ increase > or $=15 \mathrm{~mm} \mathrm{Hg}$, and PPWA decrease $>$ or $=10 \%$.Injecting the test dose resulted in an average maximum PPWA decrease by $69 \%+/-18 \%$ and $58 \%+/-14 \%$ at $79+/-22$ and $80+/-19 \mathrm{~s}$ in the 0.5 MAC and 1 MAC sevoflurane groups, respectively. The sensitivity, specificity, positive predictive, and negative predictive values for PPWA were 100\% in both sevoflurane groups, whereas by using HR and SBP criteria, the sensitivity was $90 \%$ and $95 \%$ respectively during 0.5 MAC sevoflurane anesthesia and $85 \%$ for both during 1 MAC sevoflurane anesthesia.PPWA is effective for detection of an intravascular injection of a simulated epidural epinephrine-containing test dose in pediatric patients. ${ }^{29}$

Landsverk SA, Hoiseth LO, Kvandal P, Hisdal J, Skare O, Kirkeboen KA. studied in 2008 regarding poor agreement between respiratory variations in pulse oximetry photoplethysmographic waveform amplitude and pulse pressure in intensive care unit patients.To identify fluid responsiveness, a correlation between respiratory variations in pulse pressure (DeltaPP) and respiratory variations in pulse oximetry photoplethysmographic waveform amplitude (DeltaPOP) in mechanically ventilated patients has been demonstrated. To evaluate the agreement between the two methods, knowledge about the repeatability of the methods is imperative. However, no such data exist. Based on knowledge of slow oscillation in skin blood flow, the authors hypothesized that the variability of DeltaPOP would be larger than that of DeltaPP when calculations were performed continuously over a long recording period.Respiration, continuous invasive blood pressure, pulse oximetry, and skin microcirculation were recorded in 14 mechanically ventilated intensive care unit patients. No intravenous fluid challenges were given, and no other interventions were performed during the measurements. Seventy consecutive comparisons between DeltaPP and DeltaPOP were calculated for each of the 14 patients.For all patients, DeltaPOP was $13.7+/-5.8 \%$ and DeltaPP was $5.8+/-2.6 \%(\mathrm{P}<0.001)$. There was a larger intraindividual $(8.94$ vs. $1.29 ; \mathrm{P}<0.001)$ and interindividual (26.01 vs. 5.57; $\mathrm{P}<0.001$ ) variance of DeltaPOP than of DeltaPP. In six patients, there was no significant correlation between DeltaPP and DeltaPOP. A Bland-Altman plot showed poor agreement between the two methods.A large variability of DeltaPOP and a poor agreement between DeltaPP and DeltaPOP limits DeltaPOP as a tool for evaluation of fluid responsiveness in intensive care unit patients. This is in contrast to DeltaPP, which shows a small variability. ${ }^{30}$

M. Koch1, D. De Backer2, J. L. Vincent2, L. Barvais1, D. Hennart1 and D. Schmartz studied in2008 onEffects of propofol on human microcirculationIt is increasingly believed that acute microvascular alterations may be involved in the development of organ dysfunction in critically ill patients. Propofol significantly decreases vascular tone and venous return, which can induce arterial hypotension. However, little is known about the microcirculatory effects of propofol in healthy humans. We conducted a prospective, open-labelled trial in 15 patients anaesthetized by propofol for transvaginal oocyte retrieval. 
The sublingual microcirculatory network was studied before, during, and after propofol infusion using orthogonal polarization spectral imaging. Mean (SD) calculated propofol effect-site concentration was 6.5 (1.8) $\mu \mathrm{g} \mathrm{ml}-1$. During propofol administration, systemic haemodynamic and oxygenation variables were unchanged, but total microvascular density decreased by $9.1 \%(\mathrm{P}<0.05)$. The venular density remained unchanged, but the density of perfused capillaries was significantly reduced by $16.7 \% \quad(\mathrm{P}<0.05)$. Microcirculatory alterations resolved $3 \mathrm{~h}$ after discontinuation of the propofol infusion. Propofol infusion for anaesthesia in man reduces capillary blood flow. ${ }^{31}$

Li SQ, Luo BR, Wang BG studied in 2008 onCorrelation of tip perfusion index with hemodynamics and catecholamines in patients undergoing general anesthesia].To explore the correlation of tip perfusion index (TPI) with the hemodynamics and catecholamines and to assess the value and meaning of TPI for monitoring stress responses in general anesthesia.Twenty patients who were to receive selective neurosurgery underwent induction and maintenance of propofol and remifentanil by target controlled infusion (TCI). A $60 \mathrm{~mA}-50 \mathrm{~Hz}-5$ s electrostimulus was applied before tracheal intubation and head holder setting respectively. TPI, SBP, diastolic blood pressure (DBP), heart rate (HR), and bispectral index (BIS) were recorded at nine time points: before induction (baseline), before and after electrostimulation, before and after tracheal intubation, before and after electrostimulation, and before and after head holder setting. The changes of parameters (DeltaTPI, DeltaSBP, DeltaDBP, DeltaHR, and DeltaBIS) were calculated after each stimulation. Five out of 20 patients were selected randomly to monitor the plasma concentration of catecholamine at the above time points. The responses to tracheal intubation and head holder setting were defined as the SBP increasing $>15 \mathrm{~mm} \mathrm{Hg}$ over the baseline value.DeltaTPI was significantly correlated with DeltaSBP and DeltaDBP $(r=0.623$ and $0.317, \mathrm{P}<0.01$ and 0.05$)$. The responses to tracheal intubation and head holder setting were inhibited effectively when the DeltaTPI caused by electrostimulus was less than $10 \%$ of the pre-electrostimulus TPI. TPI was significantly negatively correlated with the plasma concentrations of norepinephrine and epinephrine $(\mathrm{r}=-0.679$, and $\mathrm{r}=-0.364, \mathrm{P}<0.05$ and 0.01 ). CONCLUSION: (1) The TPI change is correlated well with the blood pressure change during stresses, and the TPI change caused by electrostimulus can predict the blood pressure change caused by tracheal intubation and head holder setting. (2) The TPI change reflects the norepinephrine plasma level indirectly. ${ }^{32}$ Reisner A1, Shaltis PA, McCombie D, Asada HH. studied in 2009 onUtility of the photoplethysmogram in circulatory monitoring. The photoplethysmogram is a noninvasive circulatory signal related to the pulsatile volume of blood in tissue and is displayed by many pulse oximeters and bedside monitors, along with the computed arterial oxygen saturation. The photoplethysmogram is similar in appearance to an arterial blood pressure waveform. Because the former is noninvasive and nearly ubiquitous in hospitals whereas the latter requires invasive measurement, the extraction of circulatory information from the photoplethysmogram has been a popular subject of contemporary research. The photoplethysmogram is a function of the underlying circulation, but the relation is complicated by optical, biomechanical, and physiologic covariates that affect the appearance of the photoplethysmogram. Overall, the photoplethysmogram provides a wealth of circulatory information, but its complex etiology may be a limitation in some novel applications. ${ }^{33}$

Korhonen I, Yli-Hankala A.studied in 2009 onPhotoplethysmography and nociception.Korhonen I, YliHankala A.Photoplethysmography (PPG), i.e. pulse oximetric wave, is a non-invasive technique that is used in anaesthesia monitoring primarily to monitor blood oxygenation. The PPG waveform resembles that of the arterial blood pressure but instead of pressure it is related to the volume changes in the measurement site and hence contains information related to the peripheral blood circulation, including skin vasomotion, which is controlled by the sympathetic nervous system. Because of this link, skin vasomotor response and PPG amplitude response have been associated with nociception under general anaesthesia. Recently, there has been interest in monitoring nociception during general anaesthesia. In many of the published studies, PPG waveform information has been included. The focus of this topical review is to provide an overview on the information embedded in the PPG waveform especially in the context of the autonomic nervous system and analgesia monitoring. ${ }^{34}$

S. Mustola ,T. Parkkari, K. Uutela, M. Huiku, M. Kymäläinen,J. Toivonen1 studied in 2010 performance of Surgical Stress Index during Sevoflurane-Fentanyl and Isoflurane-Fentanyl AnesthesiaThe performance of recently introduced Surgical Stress Index (SSI), based on heart rate and photoplethysmography, was estimated during sevoflurane-fentanyl and isoflurane-fentanyl anesthesia during surgical procedures. Forty 
ASA I-III patients were enrolled. Anesthesia was induced with fentanyl 2 and thiopentone $3-5 \mathrm{mg}$. Tracheal intubation was performed 5 minutes after fentanyl bolus. Patients were randomly allocated to receive sevoflurane () or isoflurane () in 30\% oxygen/air. State entropy was kept at 40-60, target being 50. During surgery, fentanyl boluses 1.5 were given at 30-40-minute intervals. SSI increased significantly after intubation. During surgery, the decrease of SSI after fentanyl boluses was similar in sevoflurane and isoflurane groups but SSI values were higher in sevoflurane than in isoflurane group. Tracheal intubation, skin incision, and surgical stimuli increased SSI from baseline, indicating that nociceptive stimuli increase SSI. Fentanyl boluses during surgery decreased SSI, indicating that increasing analgesia decreases SSI. ${ }^{35}$

Francesco Cresi, Emanuela Pelle, Roberto Calabrese, Luciana Costa, Daniela Farinasso and Leandra Silvestro studied in 2010 onPerfusion index variations in clinically and hemodynamically stable preterm newborns in the first week of lifeBackgroundThe perfusion index, derived from the pulse oximeter signal, seems to be an accurate predictor for high illness severity in newborns. The aim of this study was to determine the perfusion index values of clinically and hemodynamically stable preterm newborns in the first week of life.Perfusion index recordings were performed on the first, third and seventh day of life on 30 preterm newborns. Their state of health was assessed according to clinical and behaviour evaluation and to the Score for Neonatal Acute Physiology.Results- The median(interquartile range) perfusion index values were $0.9(0.6)$ on the first, 1.2(1.0) on the third, and 1.3(0.9) on the seventh day, with a significant increase between the first and the third day.Conclusions- Perfusion index proved to be an easily applicable, noninvasive method for monitoring early postnatal changes in peripheral perfusion. Its trend during the first week of life suggests that its clinical application should take age into account. Further studies are needed to obtain reference perfusion index values from a larger sample of preterm newborns, to identify specific gestational age-related cut-off values for illness and to test the role of perfusion index in monitoring critically ill neonates. ${ }^{36}$

Claudio Sandroni| Fabio Cavallaro| Cristina Marano Chiara Falcone| Paolo De Santis| Massi studies in 2010 Accuracy of plethysmographic indices as predictors of fluid responsiveness in mechanically ventilated adults: a systematic review and meta-analysisTo systematically review the accuracy of the variation in pulse oxymetry plethysmographic waveform amplitude $(\triangle \mathrm{POP})$ and the Pleth Variability Index $(\mathrm{PVI})$ as predictors of fluid responsiveness in mechanically ventilated adults. Scopus and the Cochrane Database of Systematic Reviews were screened for clinical studies in which the accuracy of $\triangle \mathrm{POP} / \mathrm{PVI}$ in predicting the hemodynamic response to a subsequent fluid bolus had been investigated. Random-effects meta-analysis was used to summarize the results. Data were stratified according to the amount of fluid bolus (large vs. small) and to the study index ( $\triangle \mathrm{POP}$ vs. PVI).Ten studies in 233 patients were included in this metaanalysis. All patients were in normal sinus rhythm. The pooled area under the receiver operating characteristic curve (AUC) for identification of fluid responders was 0.85 [95 confidence interval (CI) 0.790.92]. Pooled sensitivity and specificity were 0.80 (95\% CI 0.74-0.85) and 0.76 (0.68-0.82), respectively. No heterogeneity was found within studies with the same amount of fluid bolus, nor between studies on $\triangle \mathrm{POP}$ and those on PVI. The AUC was significantly larger in studies with a large bolus amount than in those with a small bolus [0.92 (95\% CI 0.87-0.96) vs. $0.70(0.62-0.79) ; \mathrm{p}<0.0001]$. Sensitivity and specificity were also higher in studies with a large bolus [0.84 (95\% CI 0.77-0.90) vs. $0.72(0.60-0.82)$ (small bolus), $\mathrm{p}=0.08$ and 0.86 (95 \% CI 0.75-0.93) vs. $0.68(0.56-0.77$ ) (small bolus), $\mathrm{p}=0.02$ ], respectively.Based on our meta-analysis, we conclude that $\triangle \mathrm{POP}$ and PVI are equally effective for predicting fluid responsiveness in ventilated adult patients in sinus rhythm. Prediction is more accurate when a large fluid bolus is administered. ${ }^{37}$

Sahni R, Schulze KF, Ohira-Kist K, Kashyap S, Myers MM, Fifer WP. studied in 2012 on interactions among peripheral perfusion, cardiac activity, oxygen saturation, thermal profile and body position in growing low birth weight infants. To investigate the correlation between the 'perfusion index' (PI) and other commonly used estimates of cutaneous blood flow [heart rate (HR), surface temperatures (ST) and centralto-peripheral thermal gradients (C-P grad)] and to use this new non-invasive tool to compare differences between prone and supine sleep position in low birth weight (LBW) infants.Six-hour continuous recordings of pulse oximetry, cardiac activity and absolute ST from three sites (flank, forearm and leg), along with minute-to-minute assessment of behavioural states were performed in $31 \mathrm{LBW}$ infants. Infants were randomly assigned to the prone or supine position for the first $3 \mathrm{~h}$ and then reversed for the second $3 \mathrm{~h}$. PI 
data were correlated with HR and C-P grad, and compared across sleep positions during quiet sleep (QS) and active sleep (AS).Perfusion index correlated significantly with $\mathrm{HR}(\mathrm{r}(2)=0.40)$ and flank-to-forearm thermal gradient $(\mathrm{r}(2)=0.28)$. In the prone position during QS, infants exhibited higher PI $(3.7+/-0.9$ vs. $3.1+/-0.7)$, HR (158.4 +/- 8.9 vs. $154.1+/-8.8$ bpm), $\mathrm{SpO}(2)(95.8+/-2.6$ vs. $95.2+/-2.6 \%)$, flank (36.7 +/- 0.4 vs. $36.5+/-0.4$ degrees C), forearm (36.1 +/- 0.6 vs. $35.5+/-0.4$ degrees C) and leg $(35.4+/-0.7$ vs. $34.7+/-0.7$ degrees $C)$ temperatures and narrower flank-to-forearm $(0.6+/-0.4$ vs. $0.9+/-0.3$ degrees $C)$ and flank-to-leg $(1.3+/-0.6$ vs. $1.8+/$ - 0.7 degrees $\mathrm{C})$ gradients, compared to those of the supine position. Similar differences were observed during AS.Perfusion index is a good non-invasive estimate of tissue perfusion. Prone sleeping position is associated with a higher PI, possibly reflecting thermoregulatory adjustments in cardiovascular control. The effects of these position-related changes may have important implications for the increased risk for sudden infant death syndrome in prone position. ${ }^{38}$

Xinzhong Chen,,Carsten Thee,Matthias Gruenewald, Christoph Ilies, Jan Höcker, Robert Hanss, Markus Steinfath,and Berthold Bein in 2010 studied Correlation of Surgical Pleth Index with Index with Stress Hormones during Propofol-Remifentanil AnaesthesiaEighty patients undergoing elective ear-nose-throat surgery were enrolled in the present study to investigate the relationship between surgical pleth index (SPI) and stress hormones (ACTH, cortisol, epinephrine, norepinephrine) during general anaesthesia which was induced and maintained with propofol and remifentanil using a target-controlled infusion. The study concluded that the SPI had moderate correlation to the stress hormones during general anaesthesia, but no correlation during consciousness. Furthermore, SPI values were able to predict ACTH values with high sensitivity and specificity. ${ }^{39}$

Donald H Arnold,Cathy A Jenkins, and Tina V Hartert studied on in 2010Noninvasive assessment of asthma severity using pulse oximeter plethysmograph estimate of pulsus paradoxus physiologyBackgroundPulsus paradoxus estimated by dynamic change in area under the oximeter plethysmograph waveform (PEP) might provide a measure of acute asthma severity. Our primary objective was to determine how well PEP correlates with forced expiratory volume in 1-second (\%FEV1) (criterion validity) and change of \%FEV1 (responsiveness) during treatment in pediatric patients with acute asthma exacerbations MethodsWe prospectively studied subjects 5 to 17 years of age with asthma exacerbations. PEP, \%FEV1, airway resistance and accessory muscle use were recorded at baseline and at 2 and 4 hours after initiation of corticosteroid and bronchodilator treatments. Statistical associations were tested with Pearson or Spearman rank correlations, logistic regression using generalized estimating equations, or Wilcoxon rank sum tests.ResultsWe studied 219 subjects (median age 9 years; male 62\%; African-American 56\%). Correlation of PEP with \%FEV1 demonstrated criterion validity $(\mathrm{r}=-0.44,95 \%$ confidence interval $[\mathrm{CI}],-0.56$ to $0.30)$ and responsiveness at 2 hours $(r=-0.31,95 \% \mathrm{CI},-0.50$ to -0.09$)$ and 4 hours $(\mathrm{r}=-0.38,95 \% \mathrm{CI}$, 0.62 to - 0.07). PEP also correlated with airway resistance at baseline $(r=0.28$ for ages 5 to $10 ; r=0.45$ for ages 10 to 17), but not with change over time. PEP was associated with accessory muscle use (OR 1.16, $95 \% \mathrm{CI}, 1.11$ to $1.21, \mathrm{P}<0.0001)$. ConclusionsPEPdemonstrates criterion validity and responsiveness in correlations with \%FEV1. PEP correlates with airway resistance at baseline and is associated with accessory muscle use at baseline and at 2 and 4 hours after initiation of treatment. Incorporation of this technology into contemporary pulse oximeters may provide clinicians improved parameters with which to make clinical assessments of asthma severity and response to treatment, particularly in patients who cannot perform spirometry because of young age or severity of illness. It might also allow for earlier recognition and improved management of other disorders leading to elevated pulsus paradoxus. ${ }^{40}$

Hood, Julian A.; Wilson R.; Jonathan T. studied in 2011onPleth Variability Index to Predict Fluid Responsiveness in Colorectal Surgery background: Goal-directed fluid therapy during major abdominal surgery may reduce postoperative morbidity. The Pleth Variability Index (PVI), derived from the pulse oximeter waveform, has been shown to be able to predict fluid responsiveness in a number of surgical circumstances. In the present study, we sought to determine whether PVI could predict fluid responsiveness in low-risk colorectal surgery patients who had fluid therapy guided by esophageal Doppler stroke volume measurements. Twenty-five low-risk patients undergoing colorectal resection under general anesthesia were studied. Baseline values for esophageal Doppler stroke volume and PVI taken from finger and ear probes were compared with final values after (a) a $500-\mathrm{mL}$ fluid bolus immediately after induction (steady state) and tracheal intubation before the start of the surgery, and (b) $250-\mathrm{mL}$ boluses given in response to a 
decrease in stroke volume of $10 \%$ during surgery as measured by esophageal Doppler (dynamic). Patients were classified into responders and nonresponders based on a stroke volume increase of $>10 \%$. Baseline PVI at the finger was significantly higher in responders in both steady-state and intraoperative conditions. In steady state, PVI at both finger and earlobe had significant predictive ability of an increase in stroke volume: area under the curve for finger 0.96 (95\% confidence interval $[\mathrm{CI}], 0.88-1.00 ; \mathrm{P}=0.011)$ and for earlobe 0.98 (95\% CI, 0.93-1.00; $\mathrm{P}=0.008$ ). In dynamic intraoperative conditions, PVI at the finger predicted increases in stroke volume, area under the curve $0.71(95 \% \mathrm{CI}, 0.57-0.85 ; \mathrm{P}=0.006)$, but PVI at the earlobe had no predictive value. PVI measured at the finger may be able to predict fluid responsiveness during surgery in ventilated patients. ${ }^{41}$

Mohamed H. Abdullah, , Ashraf S. Hasanin, Fatma M. Mahmou studied in 2011 Goal directed fluid optimization using Pleth variability index versus corrected flow time in cirrhotic patients undergoing major abdominal surgeriesSeveral studies have shown that hemodynamic and fluid optimization may result in improved outcome. The aim of this study was to compare between two methods of goal directed fluid optimization using protocols guided by corrected flow time (FTc) of the transesophageal doppler versus Pleth variability index (PVI group) in cirrhotic patients undergoing major abdominal surgeries.sixty cirrhotic patients Child A to B scheduled for major abdominal surgery were randomized into two groups. In both groups $500 \mathrm{~mL}$ of Ringer's acetate was infused during induction followed by a $2 \mathrm{~mL} / \mathrm{kg} / \mathrm{h}$ continuous infusion. In FTc group $(\mathrm{n}=30)$ patients with $(\mathrm{FTc})$ less than $350 \mathrm{~ms}$ were treated with bolus of fluid challenge according to a preset protocol. In PVI group $(n=30)$, PVI higher than $13 \%$ patients were given $250 \mathrm{~mL}$ of fluid bolus. There was no significant differences in the volume of crystalloids or colloids transfused to both groups with a mean value of $2670 \pm 1680 \mathrm{~mL}$ and $670 \pm 330 \mathrm{~mL}$ in the FTc guided fluid group while mean values were $2730 \pm 1760 \mathrm{~mL}$ and $690 \pm 290 \mathrm{~mL}$ in the PVI fluid guided group respectively $(\mathrm{P}>0.05)$. Also, there was no significant differences between groups regarding the intra or postoperative hemodynamic parameters. There was no significant difference regarding the overall morbidity or the hospital stay between the two groups $(\mathrm{P}>0.05)$.In conclusion, in cirrhotic patients Child A to B, FTc and PVI were considered to be adequate methods for perioperative fluid optimization, However, combination of every clinical finding, recent and conventional monitoring techniques to all haemodynamic data should be applied whenever possible. ${ }^{42}$

Bonhomme V, Uutela K, Hans G, Maquoi I, Born JD, Brichant JF, Lamy M, Hans P.studied in 2011 onComparison of the surgical Pleth Index ${ }^{\mathrm{TM}_{\text {With }}}$ haemodynamic variables to assess nociception-antinociception balance during general anaesthesia. The Surgical Pleth Index (SPI) is proposed as a means to assess the balance between noxious stimulation and the anti-nociceptive effects of anaesthesia. In this study, we compared SPI, mean arterial pressure (MAP), and heart rate (HR) as a means of assessing this balance.We studied a standard stimulus [head-holder insertion (HHI)] and varying remifentanil concentrations (CeREMI) in a group of patients undergoing neurosurgery. Patients receiving targetcontrolled infusions were randomly assigned to one of the three CeREMI (2, 4, or $\left.6 \mathrm{ng} \mathrm{m}^{-1}\right)$, whereas propofol target was fixed at $3 \mu \mathrm{g} \mathrm{ml}^{-1}$. Steady state for both targets was achieved before HHI. Intravascular volume status (IVS) was evaluated using respiratory variations in arterial pressure. Prediction probability (Pk) and ordinal regression were used to assess SPI, MAP, and HR performance at indicating CeREMI, and the influence of IVS and chronic treatment for high arterial pressure, as possible confounding factors. The maximum SPI, MAP, or HR observed after HHI correctly indicated CeREMI in one of the two patients [accurate prediction rate $(A P R)=0.5$ ]. When IVS and chronic treatment for high arterial pressure were taken into account, the APR was 0.6 for each individual variable and 0.8 when all of them predicted the same CeREMI. That increase in APR paralleled an increase in Pk from 0.63 to 0.89.SPI, HR, and MAP are of comparable value at gauging noxious stimulation-CeREMI balance. Their interpretation is improved by taking account of IVS, treatment for chronic high arterial pressure, and concordance between their predictions. $^{43}$

Elizabeth Douglas studied in 2011 on perfusion Index Used as Tool To Confirm Epidural PlacementThe perfusion index, as measured by the pulse oximeter, is a sensitive and reliable indicator of proper epidural catheter placement in obstetric patients, according to the results of studies performed at Harbor-UCLA Medical Center in Torrance, Calif. An increase in the perfusion index consistently reflected a functioning epidural catheter in patients undergoing Caesarean section, and also doubled in response to the 
administration of a standard epidural test dose in laboring parturients in two observational studies. Use of the perfusion index to confirm a functioning epidural is "a very powerful tool," Clinton Z. Kakazu, MD, told Anesthesiology News . He predicted its greatest use will be in laboring parturients."I can put an epidural in that may take five to seven minutes to work. But at two minutes I can reassure the patient, 'Within the next five minutes, your pain will go away.' I can know with confidence that the epidural will work." Dr. Kakazu currently works in private practice at Physicians' Anesthesia Service, Honolulu.The perfusion index is the first objective measure of whether an epidural catheter is functioning, said Dr. Kakazu. And confirmation of a functioning catheter occurs before the onset of sensory blockade.The ability of the perfusion index to detect the onset of epidural anesthesia derives from its sensitivity to the onset of sympathetic blockade. The perfusion index increases in response to the vasodilation resulting from the administration of the very small amount of local anesthetic contained in a standard test dose, said Dr. Kakazu's group used the Radical pulse oximeter (Masimo; Irvine, Calif.) in the study of laboring parturients and the HP model 66 oximeter (Hewlett-Packard; Andover, Mass.) in the study of patients undergoing Caesarean section. These two oximeters are among a very few with an option to display a perfusion index.

The perfusion index is calculated with proprietary algorithms. In the Masimo oximeter, the basis of the algorithm is the ratio of the fluctuating portion of light absorbance to total light absorbance, Steven $\mathrm{J}$. Barker, MD, PhD, said in an interview with Anesthesiology News. "It is not a direct measure of peripheral perfusion, but at least it is a relative measure. If perfusion index falls by a factor of two, then perfusion has probably fallen by a similar factor."

Use of the perfusion index to detect the sympathetic blockade that precedes epidural sensory block is logical, he said. Other potential applications of the perfusion index are to predict the viability of reimplanted digits or hands, to estimate volume status in trauma patients and to indicate the restoration of peripheral perfusion after cardiopulmonary bypass, suggested Dr. Barker, Department Head of Anesthesiology at the University of Arizona College of Medicine in Tucson.

Dr. Kakazu reported at the 2005 annual meeting of the American Society of Anesthesiologists that the perfusion index is a sensitive indicator of a standard epidural test dose. The investigators studied the response of the perfusion index to the administration of $3 \mathrm{~mL}$ of 1.5\% lidocaine plus 1:200,000 epinephrine in 16 laboring parturients.

The tissue perfusion index was measured with the Masimo Radical pulse oximeter by placing the probe on the patient's toe before placement of the epidural catheter (L2-3 or L3-4 and threaded 4 to $5 \mathrm{~cm}$ into the epidural space). Following a negative test dose and a 5-minute wait period, the catheter was incrementally loaded with $0.25 \%$ bupivacaine. The perfusion index was recorded every minute up to 20 minutes.

At five minutes after administration of the test dose, the perfusion index value more than doubled, from $1.11+0.88$ (mean+ standard deviation) at baseline to 2.96+2.12 ( $\mathrm{P}=0.0001$; paired t-test). At 20 minutes, the perfusion index was $7.38+3.09$ ( $\mathrm{P}<0.0001$ vs. baseline $)$.

At the annual meeting of the American Society of Anesthesiologists in 2004, Dr. Kakazu's group had reported using the HP model 66 oximeter to determine the perfusion index in 23 patients who underwent Caesarean section. Following a negative test dose, patients were administered epidural anesthesia with $2 \%$ lidocaine and 1:200,000 epinephrine delivered incrementally via an epidural catheter.

A steadily increasing perfusion index indicated a functioning epidural catheter, Dr. Kakazu reported. In contrast, a flat perfusion index characterized a failed epidural. "Toe perfusion index increased earlier in comparison to a decrease in [blood pressure]," he noted. ${ }^{44}$

Cannesson M, Desebbe O, Rosamel P, Delannoy B, Robin J, Bastien O, Lehot JJ. studied 2011 pleth variability index to monitor the respiratory variations in the pulse oximeter plethysmographic waveform amplitude and predict fluid responsiveness in the operating theatre.Respiratory variations in pulse oximetry plethysmographic waveform amplitude (DeltaPOP) can predict fluid responsiveness in mechanically ventilated patients but cannot be easily assessed at the bedside. Pleth variability index (PVI) is a new algorithm allowing for automated and continuous monitoring of DeltaPOP. We hypothesized that PVI can predict fluid responsiveness in mechanically ventilated patients under general anaesthesia.Twenty-five patients were studied after induction of general anaesthesia. Haemodynamic data [cardiac index (CI), respiratory variations in arterial pulse pressure (DeltaPP), DeltaPOP, and PVI] were recorded before and after volume expansion $(500 \mathrm{ml}$ of hetastarch $6 \%)$. Fluid responsiveness was defined as an increase in CI > 
or $=15 \%$.Volume expansion induced changes in CI [2.0 (sd 0.9) to 2.5 (1.2) litre $\min (-1) \mathrm{m}(-2)$; $\mathrm{P}<0.01]$, DeltaPOP [15 (7)\% to 8 (3)\%; P<0.01], and PVI [14 (7)\% to 9 (3)\%; P<0.01]. DeltaPOP and PVI were higher in responders than in non-responders $[19$ (9)\% vs $9(4) \%$ and 18 (6)\% vs $8(4) \%$, respectively; P<0.01 for both]. A PVI $>14 \%$ before volume expansion discriminated between responders and non-responders with $81 \%$ sensitivity and $100 \%$ specificity. There was a significant relationship between PVI before volume expansion and change in $\mathrm{CI}$ after volume expansion ( $\mathrm{r}=0.67$; $\mathrm{P}<0.01)$. PVI, an automatic and continuous monitor of DeltaPOP, can predict fluid responsiveness non-invasively in mechanically ventilated patients during general anaesthesia. This index has potential clinical applications. ${ }^{45}$

Takeyama M, Matsunaga A, Kakihana Y, Masuda M, Kuniyoshi T, Kanmura Y. studied in 2011 on impact of skin incision on the pleth variability index.The pleth variability index (PVI), which is calculated from respiratory variations in the perfusion index (PI), reportedly predicts fluid responsiveness. However, vasomotor tone fluctuations induced by nociceptive stimuli change the PI and may reduce the accuracy of PVI. The aim of this study was to confirm the effects of surgical stimuli on PVI.Twenty-four patients were examined after the induction of general anesthesia. Heart rate (HR), mean arterial blood pressure (MBP), PI, PVI, stroke volume variation (SVV), and cardiac index (CI) were recorded before and after the skin incision. PI and PVI were calculated using a Radical 7 pulse oximeter, and SVV and CI were calculated using the FloTrac/Vigileo system.

After the skin incision, the PI decreased significantly from 5.3 (4.0-6.2\%) to $3.6 \%$ (1.8-4.7\%), whereas the PVI increased significantly from 9.5 (7.0-12.0\%) to $13.5 \%$ (9.0-16.0\%). A significant negative correlation was observed between the changes in PI and PVI before and after the skin incision. The skin incision did not affect the HR, CI, or SVV but increased the MBP.This study showed a significant increase in the PVI and a negative correlation between the changes in PVI and PI before and after the skin incision. The PVI can be calculated from the variations in the PI caused not by mechanical ventilation, but rather by fluctuations in vasomotor tone. When using the PVI as an indicator for fluid responsiveness, it is crucial to pay attention to fluctuations in vasomotor tone induced by nociceptive stimuli. ${ }^{46}$

Mohamed algendi studied in 2011 on the Analysis of Fingertip Photoplethysmogram SignalsPhotoplethysmography (PPG) is used to estimate the skin blood flow using infrared light. Researchers from different domains of science have become increasingly interested in PPG because of its advantages as non-invasive, inexpensive, and convenient diagnostic tool. Traditionally, it measures the oxygen saturation, blood pressure, cardiac output, and for assessing autonomic functions. Moreover, PPG is a promising technique for early screening of various atherosclerotic pathologies and could be helpful for regular GP-assessment but a full understanding of the diagnostic value of the different features is still lacking. Recent studies emphasise the potential information embedded in the PPG waveform signal and it deserves further attention for its possible applications beyond pulse oximetry and heart-rate calculation. Therefore, this overview discusses different types of artifact added to PPG signal, characteristic features of PPG waveform, and existing indexes to evaluate for diagnoses. ${ }^{47}$

Hajime Yamazaki, Junichi Nishiyama, Toshiyasu Suzuki studied in march 182012 use of perfusion index from pulse oxymetry to determine efficacy of stellate ganglon block.Stellate ganglion block (SGB) is a widely used procedure for treatment of pain in the head and upper body, but the clinical signs used to verify the effectiveness of SGB can be ambiguous or variable in some patients. We observed the chronological changes in perfusion index (P.I.) from pulse oximetry to determine if these changes correlated with the clinical signs associated with an effective SGB. We hypothesized that PI could provide an easy method to assess the effectiveness of SGB.We compared the chronologies in PI on the treated and untreated sides of 21 patients in whom treatment by SGB was found to be effective. The SGB was performed by administering 6 $\mathrm{mL}$ of $1 \%$ mepivacaine. The effectiveness of the SGB was confirmed on the basis of clinical signs. Additionally, in two patients we tested whether increased PI on the treatment side correlated with increased microcirculation as measured by laser-Doppler blood flowmetry. On the side treated by SGB, PI increased $61.4 \%$ in the earlobe and $60.5 \%$ from baseline values in the upper limbs, at 5 minutes after initiation of the procedure. Differences in PI before and after treatment were significant at both sites. No time-course increases in PI were found on the untreated side at either site. Following SGB, increases in PI correlated with increases in blood flow as measured by laser-Doppler flowmetry.PI increased in the earlobe and upper limbs on the treated side of 21 patients who received an effective SGB but not on the untreated side. The 
positive correlations between changes in PI and both presence of clinical signs and changes in blood flow in the skin microcirculation indicate a sympatholytic effect, suggesting that the PI could be useful in determination of the efficacy of SGB. ${ }^{48}$

J. Y. Yin andK. M. Ho Studied in 2012 use of plethysmographic variability index derived from the Massimo® pulse oximeter to predict fluid or preload responsiveness: a systematic review and metaanalysisThis systematic review and meta-analysis assessed the accuracy of plethysmographic variability index derived from the Massimo ${ }^{\circledR}$ pulse oximeter to predict preload responsiveness in peri-operative and critically ill patients. A total of 10 studies were retrieved from the literature, involving 328 patients who met the selection criteria. Overall, the diagnostic odds ratio (16.0; 95\% CI 5-48) and area under the summary receiver operating characteristic curve $(0.87 ; 95 \%$ CI $0.78-0.95)$ for plethysmographic variability index to predict fluid or preload responsiveness was very good, but significant heterogeneity existed. This could be explained by a lower accuracy of plethysmographic variability index in spontaneously breathing or paediatric patients and those studies that used pre-load challenges other than colloid fluid. The results indicate specific directions for future studies. ${ }^{49}$

Hamunen K, Kontinen V, Hakala E, Talke P, Paloheimo M, Kalso E. studied 2012 effect of pain on autonomic nervous system indices derived from photoplethysmography in healthy volunteers.Photoplethysmographic pulse wave amplitude (PPGA) and heart rate (HR) can be used to measure cold, nociception-induced autonomic responses, or both. The aim of our study was to correlate the intensity of experimental pain to changes in physiological variables reflecting the autonomic nervous system response to pain.PPGA, HR, and subjective measurements of pain intensity were measured in 29 healthy male volunteers during two heat stimuli $\left(43^{\circ} \mathrm{C}\right.$ and $\left.48^{\circ} \mathrm{C}\right)$ and the cold pressor test $(\mathrm{CPT})$. Surgical pleth index (SPI), autonomic nervous system state (ANSS), and ANSS index (ANSSi) were calculated using PPGA and HR.Pain intensity scores increased on the average by $1.6,3.5$, and 8.1 for the $43^{\circ} \mathrm{C}, 48^{\circ} \mathrm{C}$, and CPT stimuli, respectively. The pain intensity scores for all three stimuli groups were significantly different from each other $(\mathrm{P}<0.001)$. All three stimuli changed HR, PPGA, SPI, ANSS, and ANSSi values significantly from their respective baseline values $(\mathrm{P}<0.001$ for all). Heat stimuli-induced pain intensity did not correlate with the magnitude of the respective changes in HR, PPGA, SPI, ANSS, and ANSSi. CPTinduced pain intensity correlated with the magnitude of the respective changes in HR, PPGA, SPI, ANSS, and ANSSi. PPGA, ANSSi, ANSS, and SPI differentiated between heat and cold stimuli-induced pain.All three thermal stimuli produced a significant change in photoplethysmograph-derived parameters. All photoplethysmograph-derived parameters appear to be suitable to study autonomic nervous system activation.Uemura A., Yagihara M., Miyabe M. studied 2013 Pulse Oximeter Perfusion Index as a Predicator for the Effect of Pediatric Epidural Block.Pediatric epidural block becomes routine for the management of pain during perioperative period. However it is difficult to evaluate the effect of pediatric epidural block non- invasively. Since the pulse oximeter perfusion index (P.I.) has been suggested to reflect the peripheral perfusion, it is hypothesized that the pulse oximeter PI might predict the effect of epidural block. Therefore we examined if the changes in P.I. reflects the effect of epidural block.Obtaining institutional approval ethics committee and written informed consent, a prospective study was operated with 50 children. They were planned to receive inguinal herniorrhaphy. After arrival at operating room, patients were monitored electrocardiography, non-invasive blood pressure, and 4 pulse oximeters (Masimo SET Radical U.S.A four limb each). Anesthesia was induced with nitrous oxide-oxygen-sevoflurane via mask. Forty patients were received one-shot lumbar epidural block. Epidural space (L2/3) was identified with drip infusion method and $0.2 \%$ ropivacaine $(0.7 \mathrm{ml} / \mathrm{kg})$ was administered. PI was recorded with Physiolog®(Masimo Corp. U.S.A). Non-invasive blood pressure, heart rate, endtidal $\mathrm{CO}_{2}$, endtidal Sevo\% and respiratory rate were also recorded. Ten patients were not performed epidural block and administered acetoaminophen suppository114 min after one shot lumbar epidural injection, PI values of both lower limb were significantly increased compared with both upper $\operatorname{limb}(\mathrm{P}<0.05)<$ Figure $>$. Failed epidural block (elevated heart rate, respiratory rate and movement after incision) showed low PI of lower limb. Without epidural block group, PI also remained low in every limb.

Failed epidural block or without epidural block did not affect the PI values. The pulse oximeter PI reflects the peripheral perfusion and changed by epidural block, PI value can be used as a prediction for the effect of 
epidural block. As we use pulse oximeter routinely in every patients during operation, PI value is useful, objective, and non-invasive method to evaluate the effect of epidural block in pediatric patients. ${ }^{50}$

Sahni R. studied in 2012 on noninvasive monitoring by photoplethysmographyThe photoplethysmogram (PPG) is a noninvasive circulatory signal related to the pulsatile volume in tissue and is displayed by many pulse oximeters. The PPG is similar in appearance to the invasive arterial waveform, but is noninvasive and ubiquitous in hospitals. There is increasing interest in seeking circulatory information from the PPG and developing techniques for a wide variety of novel applications. This article addresses the basic physics of photoplethysmography, physiologic principles behind pulse oximetry operation, and recent technological advances in the usefulness of the PPG waveform to assess microcirculation and intravascular fluid volume monitoring during intensive care. ${ }^{51}$

Jung-Nam Kwon and Whan-Bong Lee studied in 2012 onUtility of digital pulse oximetry in the screening of lower extremity arterial diseaseThe aim of this study was to evaluate screening methods in the lower extremities by measurement of the digital pulse oximetry (oxygen percent saturation $\left[\mathrm{SpO}_{2}\right]$ ) of toes for peripheral arterial disease (PAD).A prospective study was performed among 49 patients (98 limbs) with lower extremity arterial occlusive disease. We attempted to measure the ankle-brachial index (ABI), digital pulse oximetry $\left(\mathrm{SpO}_{2}\right)$, and computerized tomographic angiography (CTA). Patients were divided into three groups by the traditional Fontaine classification system by symptom and CTA criteria: 1) Critical limb ischemia (Fontaine III and IV), 2) Claudication; (Fontaine II), and 3) asymptomatic limbs (Fontaine I). The sensitivity, specificity, positive and negative predictive values between active treatment groups (group I and II; endovascular and open surgery) and conservative group (group III) are all statistically significant. ABI; $55.09 \%, 94 \%, 96.7 \%, 39.02 \%(\mathrm{R}=12.54, \mathrm{P}<0.000) \mathrm{SpO}_{2} ; 87.06 \%, 87.8 \%, 84.3 \%, 90 \%(\mathrm{R}=40.11, \mathrm{P}<$ 0.000). Pre- $\mathrm{SpO}_{2}$ and pre-ABI all show statistically significant correlation in group I vs. group II, symptomatic PAD (group I and II) vs. asymptomatic PAD (group III), and the total PAD comparison. The Pearson's correlation coefficient between SpO2 and ABI all show significant correlation in group II. Pre$\mathrm{SpO}_{2}$ vs. Pre-ABI show strong positive correlation except asymptomatic group (group III).Digital pulse oximetry can be a useful, simple, noninvasive screening device as well as ABI in PAD. ${ }^{52}$

Toyama S, Kakumoto M, Morioka M, Matsuoka K, Omatsu H, Tagaito Y, Numai T, Shimoyama M.Perfusion index studied in 2013 on perfusion index derived from a pulse oximeter can predict the incidence of hypotension during spinal anaesthesia for Caesarean delivery.tHypotension during spinal anaesthesia for Caesarean delivery is a result of decreased vascular resistance due to sympathetic blockade and decreased cardiac output due to blood pooling in blocked areas of the body. Change in baseline peripheral vascular tone due to pregnancy may affect the degree of such hypotension. The perfusion index (P.I.) derived from a pulse oximeter has been used for assessing peripheral perfusion dynamics due to changes in peripheral vascular tone. The aim of this study was to examine whether baseline P.I. could predict the incidence of spinal anaesthesia-induced hypotension during Caesarean delivery.Parturients undergoing elective Caesarean delivery under spinal anaesthesia with hyperbaric bupivacaine $10 \mathrm{mg}$ and fentanyl $20 \mu \mathrm{g}$ were enrolled in this prospective study. The correlation between baseline PI and the degree of hypotension during spinal anaesthesia and also the predictability of spinal anaesthesia-induced hypotension during Caesarean delivery by P.I. were investigated.Baseline P.I. correlated with the degree of decreases in systolic and mean arterial pressure $(r=0.664, \mathrm{P}<0.0001$ and $\mathrm{r}=0.491, \mathrm{P}=0.0029$, respectively). The cut-off P.I. value of 3.5 identified parturients at risk for spinal anaesthesia-induced hypotension with a sensitivity of $81 \%$ and a specificity of $86 \%(\mathrm{P}<0.001)$. The change of P.I. in parturients with baseline P.I. $\leq 3.5$ was not significant during the observational period, while PI in parturients with baseline PI $>3.5$ demonstrated marked decreases after spinal injection.We demonstrated that higher baseline PI was associated with profound hypotension and that baseline PI could predict the incidence of spinal anaesthesia-induced hypotension during Caesarean delivery. ${ }^{53}$

Kinoshita M, Hawkes CP, Ryan CA, Dempsey EM. studied in 2013 on perfusion index in the very preterm infant.Perfusion Index (P.I.) is a quantifiable measurement of peripheral perfusion and may be a useful adjunct to the assessment of circulatory status in the newborn. (i) To assess reproducibility of P.I. and (ii) To determine whether there is a difference between simultaneously obtained limb measurements of P.I. in newborns $<32$ weeks GA in the transitional period.Perfusion Index was measured in newborns <32 weeks during the first $48 \mathrm{~h}$ of life. To examine reproducibility, the pulse oximetry probe was replaced on the same 
limb consecutively by the same operator. Upper and lower limbs were then simultaneously evaluated over a 5-min period. Heart rate, blood pressure, birth weight, ventilation requirement, inotrope use, lactate, $\mathrm{PCO}_{2}$ and CRIB-II score were also recorded.Thirty infants were assessed. Intraclass correlation coefficient for reproducibility in the same limb was high $(\mathrm{r}$ value $=0.982 \mathrm{p}<0.001)$. Measurements obtained in the right upper limb were consistently higher than either lower limb. The median (IQR) PI for the entire cohort was 0.70 (0.29-1.35). No correlation existed between gestational age, birth weight, CRIB scores, systolic and diastolic blood pressure, mean blood pressure and median PI values.Perfusion Index measurement is reproducible, and values are highest in the right upper limb. Wide differences between right upper and lower limb readings are most likely related to transitional circulatory changes. ${ }^{54}$

ZHANG Yan-ming,XUN Bin,CHEN Bin,et al studied in 2013 on the clinical application in preventing laparoscopic harmful stimulation using fentanyl with butorphanolObjective:To compare the prevention and control of harmful stimulation when fentanyl with butorphanol and fentanyl were used for gynecological laparoscopic surgery.Methods:There were 100 cases scheduled for general anesthesia,gynecological laparoscopy patients,ASA I or grade $I I$, aged from 18 to 45 years old, who were randomly divided into two groups,the fentanyl with butorphanol group(BF) and fentanyl(F) group.The patients going into operation room would be given the ECG,noninvasiveblood pressure,SPO2,PI,the BIS monitoring.Patients were observed before induction(T0), after induction before intubation(T1),endotracheal intubation(T2),skin incision(T3), $\mathrm{CO}_{2}$ pneumoperitoneum(T4),pulling the peritoneum(T5),suction(T6), extubation(T7) at each time point,BP and $\mathrm{HR}, \mathrm{SPO}_{2}$, the BIS,PI;the BIS, breathing recovery time,time to eye opening, extubation time, and RSS, the VAS, the RS score after surgery;induction period's choke cough and 24 hours postoperative nausea and vomiting,hoar seness,sore throat occurrence.Results:BF patients in intubation,incision,pulling the peritoneum, $\mathrm{CO}_{2}$ gas abdomen and other strong harmful stimulation.Heart rate,mean arterial pressure compared with the induction of anesthesia although elevated,the P0.05;peripheral perfusion index compared with induction of anesthesia decreased slightly,but P0.05,bispectral index stabilized at 40 45.After surgery the BIS values of BF group is lower than the F,P0.05.RSS score,RS score,VAS score,the BF group was lower than the F,P0.05.Breathing recovery time,time to eye opening,extubation time,the BF group was much shorter than those in Group F,P0.05. The difference was statistically significant.Conclusion:Fentanyl with butorphanol in gynecological laparoscopy under general anesthesia can effectively inhibit the harmful stimulation caused by surgery and anesthesia to maintain stable vital signs;can recover quickly after surgery,restore satisfactory spontaneous breathing and orientation.Because of residual analgesic effects,patients will have no abdominal pain,bloating,nausea,vomiting and other discomforts, have no additional postoperative analgesia pump,but also have no cost of treatment of adverse reactions.Compared with traditional opioid fentanyl alone,fentanyl with butorphanol has obvious advantage. ${ }^{55}$

Zifeng Xu, Jianhai Zhang, Hao Shen, and Jijian Zheng in 2013 studied Assessment of Pulse Oximeter Perfusion Index in Pediatric Caudal Block under Basal Ketamine Anesthesia. Whether pulse oximeter perfusion index (PI) may be applied to detect the onset of caudal block in pediatric patients under ketamine intravenous basal anesthesia is investigated. 40 ASA I, 2-8-year-old boys scheduled for elective circumcision surgery were randomized into two groups. Group I: 20 patients were anesthetized by $2 \mathrm{mg} \cdot \mathrm{kg}-1$ ketamine intravenous injection (IV) followed by caudal block using $1 \mathrm{~mL} \cdot \mathrm{kg}-1$ lidocaine $(1 \%)$; Group II: 20 patients were anesthetized by $2 \mathrm{mg} \cdot \mathrm{kg}-1$ ketamine IV only. PI on the toe in Group II decreased by $33 \pm 12 \%, 71 \pm 9 \%$ and $65 \pm 8 \%$ at $1 \mathrm{~min}, 15 \mathrm{~min}$, and $30 \mathrm{~min}$ after ketamine injection. The maximum increase in MAP and HR after ketamine IV was $11 \pm 6 \%$ at $3 \mathrm{~min}$ and $10 \pm 6 \%$ at $2 \mathrm{~min}$. Compared to the PI value before caudal injection of lidocaine, PI in Group I increased by $363 \pm 318 \%$ and $778 \pm 578 \%$ at $5 \mathrm{~min}$ and 20 min after caudal block, while no significant changes in MAP and HR were found compared to the baseline before caudal block. Thus, PI provides an earlier, more objective, and more sensitive indicator to assess the early onset of caudal block under basal ketamine anesthesia. ${ }^{56}$

Michał Kowalczyk, Anna Fijałkowska, Andrzej Nestorowicz studied in 2013 New generation pulse oximetry in the assessment of peripheral perfusion during general anaesthesia - a comparison between propofol and desflurane background: A pulse oximeter is a standard device for perioperative monitoring. It is well known that the early detection of tissue hypoxia is of great importance. It has been made easier due to a new generation pulse oximetry device from Masimo. This enables measurements of the peripheral 
perfusion index (P.I.) in real time. It has been found that volatile anaesthetics such as sevoflurane and desflurane increase the perfusion index. As we know, no data is available about perfusion index during propofol/remifentanil total intravenous anaesthesia.

Methods: ASA I and II class women scheduled for elective gynaecological surgery were eligible for the study. Patients were divided into two groups: group $\mathrm{P}$ receiving propofol/remifentanil intravenous anaesthesia and group D receiving desflurane/fentanyl general anaesthesia. PI was noted before anaesthesia, after remifentanil/fentanyl injection, after endotracheal intubation, at the beginning of surgery, during the procedure at ten minute intervals, at the end of the procedure, after awakening, after extubation and before discharge to the ward.

Results: Eighty-three patients were enrolled to the study. In both groups, P.I. increased significantly from the start to the end of surgery. There was a significant correlation between P.I. and end-tidal desflurane concentration $(\mathrm{r}=0.807 ; \mathrm{P}=0.001)$. No correlation was found between propofol or remifentanil concentrations and P.I.

Conclusion: Both intravenous propofol/remifentanil and desflurane/fentanyl general anaesthesia increase peripheral perfusion. An increase in end-tidal desflurane concentration raises peripheral perfusion. ${ }^{57}$

van Genderen ME, Bartels SA, Lima A, Bezemer R, Ince C, Bakker J, van Bommel J. studied in 2013 peripheral perfusion index as an early predictor for central hypovolemia in awake healthy volunteers.In healthy volunteers, we investigated the ability of the pulse oximeter-derived peripheral perfusion index (PPI) to detect progressive reductions in central blood volume.Twenty-five awake, spontaneously breathing, healthy male volunteers were subjected to progressive reductions in central blood volume by inducing stepwise lower body negative pressure (LBNP) with $20 \mathrm{~mm} \mathrm{Hg}$ for 5 minutes per step, from 0 to $-20,-40$, 60 , and back to $0 \mathrm{~mm} \mathrm{Hg}$. Throughout the procedure, stroke volume (SV), heart rate (HR), and mean arterial blood pressure were recorded using volume-clamp finger plethysmography. Assessment of the PPI was done by pulse oximetry. Additionally, the forearm-to-fingertip skin-temperature gradient was measured. Data are presented as mean \pm SE. PPI underwent $\log$ transformation and is presented as median (25th-75th). Of the 25 subjects, one did not complete the study because of cardiovascular collapse. After the first LBNP step (-20 $\mathrm{mm} \mathrm{Hg}$ ), PPI decreased from $2.2(1.6-3.3)$ to $1.2(0.8-1.6)(\mathrm{P}=0.007)$ and $\mathrm{SV}$ decreased from $116 \pm 3.0 \mathrm{~mL}$ to $104 \pm 2.6 \mathrm{~mL}(\mathrm{P}=0.02)$. The magnitude of the PPI decrease $(41 \% \pm 6.0 \%)$ was statistically different from that observed for SV $(9 \% \pm 1.3 \%)$ and HR $(3 \% \pm 1.9 \%)$. During progression of LBNP, SV decreased and HR increased progressively with the increased applied negative pressure, whereas the PPI remained low throughout the remainder of the protocol and returned to baseline values when LBNP was released. At -60 $\mathrm{mm}$ Hg LBNP, SV decreased and HR increased by $36 \% \pm 0.9 \%$ and $33 \% \pm 2.4 \%$ from baseline, respectively. Mean arterial blood pressure remained in the same range throughout the experiment.

These results indicate that the pulse oximeter-derived PPI may be a valuable adjunct diagnostic tool to detect early clinically significant central hypovolemia, before the onset of cardiovascular decompensation in healthy volunteers. ${ }^{58}$

Hosam M Atef, Salah Abd Fattah, Mohammed Emad Abd Gaffer, and Ahamed Abd Al Rahman in 2013 studied Perfusion index versus non-invasive hemodynamic parameters during insertion of i-gel, classic laryngeal mask airway and endotracheal tubePerfusion index (P.I.) is a non-invasive numerical value of peripheral perfusion obtained from a pulse oximeter. In this study, we evaluated the efficacy of P.I. for detecting hemodynamic stress responses to insertion of i-gel, laryngeal mask airway (LMA) and endotracheal tube and compare, its reliability with the conventional haemodynamic criteria in adults during general anaesthesia.Sixty patients scheduled for elective general surgery under general anaesthesia were randomised to three groups. (i-gel, LMA and ET groups ( $n=20 /$ group). Heart rate (HR) (positive if $\geq 10$ bpm), systolic blood pressure (SBP), diastolic blood pressure (DBP) (positive if $\geq 15 \mathrm{~mm} \mathrm{Hg}$ ) and PI (positive if $\leq 10 \%$ ) were monitored for 5 min after insertion. Main outcome measures: SBP, DBP, HR and PI were measured before induction of anaesthesia and before and after insertion of the airway device.Insertion of airway devices produced significant increases in HR, SBP and DBP in LMA and ET groups. Moreover, PI was decreased significantly by $40 \%, 100 \%$ and $100 \%$ in the three groups. Using the PI criterion, the sensitivity was 100\% (CI 82.4-100.0\%). Regarding the SBP and DBP criterions, the sensitivity was $44.4 \%$ (CI 24.6-66.3\%), 55.6\% (CI 33.7-75.4\%) respectively. Also, significant change in the mean PI over time (from pre-insertion value to the $1 \mathrm{st} \mathrm{min}$, 3rd min, until the 4th min after insertion without regard the device 
type), $(\mathrm{P}<0.001) . \mathrm{PI}$ is a reliable and easier alternative to conventional haemodynamic criteria for detection of stress response to insertion of i-gel LMA and ET during propofol fentanyl isoflurane anaesthesia in adult patients. $^{59}$

Chiharu Kuroki, Kohei Godai, Maiko Hasegawa-Moriyama, Tamotsu Kuniyoshi, Akira Matsunaga, Yuichi Kanmura, Tomoyuki Kuwaki studied in 2013Perfusion index as a possible predictor for postanesthetic shivering. Postanesthetic shivering can be triggered by surgical stress and several aspects of anesthetic management and is frequently preceded by a decrease in peripheral blood flow due to thermoregulatory vasoconstriction. As perfusion index correlates with peripheral blood flow, we examined whether perioperative perfusion index, measured using pulse oximetry, might be correlated with postanesthetic shivering.

Twenty-eight patients presenting for elective abdominal surgery were enrolled. Core (esophagus) and peripheral (finger) temperatures and perfusion index were recorded in the perioperative periods. Correlations between perfusion index and peripheral temperature and core-to-peripheral temperature gradient were then explored. Plasma levels of epinephrine and norepinephrine were also measured. The extent of shivering was graded after emergence from anesthesia.

Perfusion index declined before emergence from anesthesia in patients who then developed postanesthetic shivering. This coincided with the time at which the difference between core and peripheral temperature became dissociated and peripheral temperature declined. Perioperative perfusion index was correlated with peripheral temperature and peripheral-core temperature gradient. Perfusion index at closure of the peritoneum predicted postanesthetic shivering and was significantly correlated with the extent of shivering. Plasma levels of both epinephrine and norepinephrine were significantly elevated after shivering events.Perfusion index was significantly lower in patients with postanesthetic shivering before emergence from anesthesia, indicating that measurement of perfusion index during and before the end of anesthesia might be a useful means of predicting postanesthetic shivering. ${ }^{60}$

Toshiki Nishimura,Aya Nakae,Masahiko Shibata, Takashi Mashimo, and Yuji Fujinoin 2014 studied Agerelated and sex-related changes in perfusion index in response to noxious electrical stimulation in healthy subjectsEven though pain is a subjective phenomenon, its objective evaluation in humans is important because subjects requiring pain evaluation may be unable to describe their pain intensity because of decreased awareness or impaired cognitive function. Previous reports indicate that the perfusion index (PI), which is calculated from pulse oximeter waveforms, has some utility in assessing pain. However, ageassociated and sex-associated differences in change of PI have hitherto not been evaluated for assessment of pain. Therefore, we aimed to estimate the utility of age-related differences in PI change among healthy volunteers subjected to electrical stimulation.We measured PI and pulse rate in 70 healthy volunteers exposed to gradually increasing electrical stimulation. The subjects were classified into four groups, ie, young men, young women, aged men, and aged women. Stimulation was stopped when subjects reached their pain tolerance threshold. The average PI and pulse rate were calculated 10 seconds before and after electrical stimulation and compared across the four groups. Changes in PI and pulse rate were analyzed using the paired t-test.The PI was significantly decreased in response to pain stimulation in young men $(\mathrm{P}<0.0001)$, young women $(\mathrm{P}=0.0002)$, and aged men $(\mathrm{P}=0.0158)$. However, aged women failed to show significant changes in PI before or after stimulation. The pulse rate was not significantly altered in any of the groups.PI may be an independent parameter reflecting the perception of noxious stimuli and could be used for objective evaluation of pain perception in healthy volunteers, except when it is used for pain evaluation in elderly women. ${ }^{61}$

Loupec T, Nanadoumgar H, Frasca D, Petitpas F, Laksiri L, Baudouin D, Debaene B, Dahyot-Fizelier C, Mimoz OPleth studied in 2014 variability index predicts fluid responsiveness in critically ill patients.To investigate whether the pleth variability index, a noninvasive and continuous tool, can predict fluid responsiveness in mechanically ventilated patients with circulatory insufficiency.It was aProspective study.In Surgical intensive care unit of a university hospital.Forty mechanically ventilated patients with circulatory insufficiency in whom volume expansion was planned by attending physician. Exclusion criteria included spontaneous respiratory activity, cardiac arrhythmia, known intracardiac shunt, severe hypoxemia (Pao2/Fio2 $<100 \mathrm{~mm} \mathrm{Hg}$ ), contraindication for passive leg raising, left ventricular ejection fraction of $<50 \%$, and hemodynamic instability during the procedure.Fluid challenge with $500 \mathrm{~mL}$ of 130/0.4 hydroxyethyl- 
starch if respiratory variations in arterial pulse pressure were $\geq 13 \%$ or with passive leg raising if variations in arterial pulse pressure were $<13 \%$.Pleth variability index, variations in arterial pulse pressure, and cardiac output estimated by echocardiography were recorded before and after fluid challenge. Fluid responsiveness was defined as an increase in cardiac output of $\geq 15 \%$. Twenty-one patients were responders and 19 were nonresponders. Mean \pm sd pleth variability index $(28 \% \pm 13 \%$ vs. $11 \% \pm 4 \%)$ and arterial pulse pressure variation $(22 \% \pm 11 \%$ vs. $5 \% \pm 2 \%)$ values at baseline were significantly higher in responders than in nonresponders. The pleth variability index threshold value of $17 \%$ allowed discrimination between responders and nonresponders with a sensitivity of $95 \%$ (95\% confidence interval, $74 \%$ to $100 \%$ ) and a specificity of $91 \%$ (95\% confidence interval, $70 \%$ to $99 \%$ ). The pleth variability index at baseline correlated $(\mathrm{r}=.72, \mathrm{p}<.0001)$ with the percentage change in cardiac output induced by fluid challenge, suggesting that a higher pleth variability index at baseline will correlate with a higher percentage change in cardiac output after volume expansion. The pleth variability index can predict fluid responsiveness noninvasively in intensive care unit patients under mechanical ventilation. ${ }^{62}$

Hakan N1, Dilli D, Zenciroglu A, Aydin M, Okumus N. studied on Reference values of perfusion indices in hemodynamically stable newborns during the early neonatal period in2014 they aimed to determine reference values of perfusion index (PI) in healthy newborns during the early neonatal period. Preductal (right hand) and postductal (foot) PI values were assessed during the first 5 days of life by using a new generation pulse oximetry. A total of 241 newborn infants (196 [81.3\%] term and 45 [18.7\%] preterm) were enrolled to the study. On the first day, in term infants, the median (interquartile range [IQR]) preductal and postductal PI were $1.35(1.02-1.91)$ and $0.88(0.62-1.22)$, respectively $(\mathrm{p}=0.001)$. These values were $0.88(0.60-1.26)$ and $0.61(0.35-0.92)$ in preterm infants, with the same respect $(p=0.001)$. From the first to third days, preductal PI remained significantly higher than the postductal PI ( $<<0.001$, for all comparisons). Both preductal and postductal PI of term newborns were significantly higher than those of preterm infants ( $p<0.001$, for both comparisons). These differences in PI disappeared on the fifth day of life. It was Concluded PI values which reflect peripheral perfusion seem to reach to a steady state on the fifth day of life following physiological maturation. ${ }^{63}$

Xu Z1, Zhang J2, Xia Y2, Deng X3Accuracy of pulse oximeter perfusion index in thoracic epidural anesthesia under basal general anesthesia.To observe the change of PVI after thoracic epidural block on the basis of general anesthesia.in 26 patients undergoing elective upper abdominal operations, changes of SVI, PVI, SVV, PPV and CVP were monitored immediately before and 10 minutes after T8-9 thoracic epidural anesthesia on the basis of general anesthesia. The definition was that patients with $\Delta$ SVI greater than $10 \%$ belonged to response group to epidural block.

Before epidural block, the PVI, SVV and PPV baseline values in patients of response group were significantly higher than those in patients of non-response group. PVI, SVV and PPV after epidural block were significantly higher than immediately before epidural block $(\mathrm{P}<0.001)$. PVI, SVV and PPV baseline values immediately before epidural block were positively correlated with $\triangle \mathrm{SVI}$; the correlation coefficients were $0.70,0.71$ and 0.63 , respectively, $\mathrm{P} \leq 0.001$. The optimal critical values for PVI, SVV and PPV to predict response to T8-9 gap epidural block under general anesthesia were $16 \%$ (sensitivity $80 \%$, specificity $92 \%$ ), $13 \%$ (sensitivity $90 \%$, specificity $62 \%$ ) and $12 \%$ (sensitivity $90 \%$, specificity $77 \%$ ), respectivelly.PVI can be used as a noninvasive indictor to monitor volume change after thoracic epidural block on the basis of general anesthesia ${ }^{64}$

van Genderen ME, Paauwe J, de Jonge J, van der Valk RJ, Lima A, Bakker J, van Bommel J. studied in 2014 clinical assessment of peripheral perfusion to predict postoperative complications after major abdominal surgery early: a prospective observational study in adults.Altered peripheral perfusion is strongly associated with poor outcome in critically ill patients. We wanted to determine whether repeated assessments of peripheral perfusion during the days following surgery could help to early identify patients that are more likely to develop postoperative complications.

Haemodynamic measurements and peripheral perfusion parameters were collected one day prior to surgery, directly after surgery (D0) and on the first (D1), second (D2) and third (D3) postoperative days. Peripheral perfusion assessment consisted of capillary refill time (CRT), peripheral perfusion index (PPI) and forearmto-fingertip skin temperature gradient (T(skin-diff)). Generalized linear mixed models were used to predict severe complications within ten days after surgery based on Clavien-Dindo classification.We prospectively 
followed 137 consecutive patients, from among whom 111 were included in the analysis. Severe complications were observed in 19 patients (17.0\%). Postoperatively, peripheral perfusion parameters were significantly altered in patients who subsequently developed severe complications compared to those who did not, and these parameters persisted over time. CRT was altered at D0, and PPI and T(skin-diff) were altered on D1 and D2, respectively. Among the different peripheral perfusion parameters, the diagnostic accuracy in predicting severe postoperative complications was highest for CRT on D2 (area under the receiver operating characteristic curve $=0.91(95 \%$ confidence interval $(\mathrm{CI})=0.83$ to 0.92$)$ ) with a sensitivity of $0.79(95 \% \mathrm{CI}=0.54$ to 0.94$)$ and a specificity of $0.93(95 \% \mathrm{CI}=0.86$ to 0.97$)$. Generalized mixed-model analysis demonstrated that abnormal peripheral perfusion on D2 and D3 was an independent predictor of severe postoperative complications $(\mathrm{D} 2$ odds ratio $(\mathrm{OR})=8.4,95 \% \mathrm{CI}=2.7$ to $25.9 ; \mathrm{D} 2 \mathrm{OR}=$ $6.4,95 \% \mathrm{CI}=2.1$ to 19.6 ). In a group of patients assessed following major abdominal surgery, peripheral perfusion alterations were associated with the development of severe complications independently of systemic haemodynamics. Further research is needed to confirm these findings and to explore in more detail the effects of peripheral perfusion-targeted resuscitation following major abdominal surgery. ${ }^{65}$

Joana Jardim, Ruben Rocha, Gorett Silva, Hercília Guimarães studied in 2014 onPeripheral perfusion indexreference range in healthy Portuguese term newborns

Abstract Peripheral perfusion index (PPI) is a non-invasive numerical value of peripheral perfusion derived from a pulse oximeter signal. It has been suggested that PPI may be a valuable adjunct diagnostic tool to detect early clinically significant hemodynamic embarrassment. The aim of this paper was to determine normal PPI in healthy newborns, in order to establish cut-off values that can be use in different pathologic settings. Prospective observational study performed with term newborns, born in a tertiary level care hospital between January 1st to December 31st 2012. Demographic data such as gender, gestational age, birth weight and Apgar score were collected. PPI, heart rate (HR) and arterial blood oxygen saturation (SpO2) were evaluated simultaneous on the right hand (preductal) and on the left foot (postductal) of the newborn, before discharge from the hospital. 2,032 newborns, 52\% male, with a mean birth weight of 3,237 $\pm 432 \mathrm{~g}$ and median gestational age of 39 weeks, were evaluated. PPI values obtained were: preductal median of 1.6 with interquartile range of 1.2-2.3, postductal median of 1.4 with interquartile range of 1-2 (p $<0.001)$. PPI is an easily applicable non invasive method to monitor peripheral perfusion changes. We established normal PPI values in healthy Portuguese newborns. PPI was higher on the upper limb (preductal) when compared to the lower limb (postductal).This finding has important implications, in the time of choosing a single probe placement, and in the interpretation of the results. ${ }^{66}$

Huai-wu He,Da-wei Liu, Yun Long, and Xiao-ting Wang studied 2015 the peripheral perfusion index and transcutaneous oxygen challenge test are predictive of mortality in septic patients after resuscitationThe peripheral perfusion index (PI) is a noninvasive numerical value of peripheral perfusion, and the transcutaneous oxygen challenge test (OCT) is defined as the degree of transcutaneous partial pressure of oxygen $\left(\mathrm{PtcO}_{2}\right)$ response to $1.0 \mathrm{FiO}_{2}$. The value of noninvasive monitoring peripheral perfusion to predict outcome remains to be established in septic patients after resuscitation. Moreover, the prognostic value of PI has not been investigated in septic patients.Forty-six septic patients, who were receiving PiCCO-Plus cardiac output monitoring, were included in the study group. Twenty stable postoperative patients were studied as a control group. All the patients inspired 1.0 of $\mathrm{FiO}_{2}$ for 10 minutes during the OCT. Global hemodynamic variables, traditional metabolic variables, PI and OCT related-variables were measured simultaneously at 24 hours after PiCCO catheter insertion. We obtained the 10min-OCT ((PtcO2 after 10 minutes on inspired 1.0 oxygen) - (baseline $\mathrm{PtcO} 2))$, and the oxygen challenge index $\left((10 \mathrm{~min}-\mathrm{OCT}) /\left(\mathrm{PaO}_{2}\right.\right.$ on inspired 1.0 oxygen - baseline $\left.\mathrm{PaO}_{2}\right)$ ) during the OCT.The PI was significantly correlated with baseline $\mathrm{PtcO} 2,10 \mathrm{~min}-\mathrm{OCT}$ and oxygen challenge index (OCI) in all the patients. The control group had a higher baseline $\mathrm{PtcO}_{2}, 10 \mathrm{~min}-\mathrm{OCT}$ and PI than the septic shock group. In the sepsis group, the macro hemodynamic parameters and $\mathrm{ScvO}_{2}$ showed no differences between survivors and nonsurvivors. The nonsurvivors had a significantly lower PI, 10min-OCT and OCI, and higher arterial lactate level. The PI, 10min-OCT and OCI predicted the ICU mortality with an accuracy that was similar to arterial lactate level. A PI $<0.2$ and a $10 \mathrm{~min}-\mathrm{OCT}<66 \mathrm{mmHg}$ were related to poor outcome after resuscitation.The PI and OCT are predictive of mortality for septic patients after resuscitation. Further investigations are required to 
determine whether the correction of an impaired level of peripheral perfusion may improve the outcome of septic shock patients. ${ }^{67}$

Mousa WF studied in 2015 effect of hypercapnia on pleth variability index during stable propofol: Remifentanil anesthesia.The pleth variability index (PVI), which is calculated from respiratory variations in the perfusion index (P.I.), has been shown to predict fluid responsiveness in mechanically ventilated patients; however, vasomotor tone changes induced by hypercapnia can affect PI and hence may slim down the accuracy of PVI. This study was designed to find out the impact of mild hypercapnia on PVI.

A total of 30 patients were randomized after induction of general anesthesia with target controlled infusion propofol and remifentanil to either hypercapnia, (etCO2 $=45 \mathrm{mmHg}$ ), (group 1, 15 patients) or normocapnia (etCO $2=35 \mathrm{mmHg}$ ) (group 2, 15 patients). After a stabilization period of $10 \mathrm{~min}$, patients were crossed over to the other intentional level of etCO $\mathrm{C}_{2}$. Heart rate (HR), mean arterial pressure (MAP), PI, PVI were collected at the end of each stabilization period.

Patient characteristics and baseline values of HR, MAP, PI and PVI were comparable between the groups. Carryover effect was statistically excluded. Hypercapnia significantly increased PI and decreased PVI with significant negative correlation.Hypercapnia retracts back PVI values compared with normocapnia. Precise judgment of fluid responsiveness as indicated by PVI necessitates its comparison against similar etCO ${ }_{2}$ levels. ${ }^{68}$

Yukihiro Sawada Gohichi Tanaka studied in 2015 Normalized pulse volume (NPV) derived photoplethysmographically as a more valid measure of finger vascular toneNormalized pulse volume (NPV) was advocated as a more valid measure for the assessment of finger vascular tone. Based on the optical model in the finger tip expressed by Lambert--Beer's law, NPV is expressed as Delta I(a)/I. Here, Delta I(a) is the intensity of pulsatile component superimposed on the transmitted light (I). Theoretically, NPV seems to be superior to the conventional pulse volume (PV; corresponding to Delta I(a)). Firstly, NPV is in direct proportion to Delta $\mathrm{V}(\mathrm{a})$, which is the pulsatile component of the arterial blood volume, in a more exact manner. Relatedly, NPV can be processed as if it is an absolute value. Secondly, the sensitivity of NPV during stressful stimulations is expected to be higher. These expectations were supported experimentally using 13 male students. Firstly, the correlation between cutaneous vascular resistance in the finger tip (CVR) and NPV was higher than that between CVR and PV among all the subjects, although there was not much difference between these correlations within each subject. Secondly, NPV decreased much more than PV during mental stress. Some limitations of the present study were addressed, including the point that certain factors can violate the direct proportional relationship of NPV and PV to Delta V(a). ${ }^{69}$

Recent advance in patient monitoring Photoplethysmographic waveform variation is used to detect volume changes in the microvascular bed of tissue and is also a reliable monitor of changes od sympathetic function .Perfusion index derived from photoplethysmography waveform amplitude .In patient ventilated with tidal volume over $8 \mathrm{ml} / \mathrm{kg}$ under sedation, pleth variability index can detect hemodynamic effect o positive end expiratory pressure. ${ }^{70}$

\section{MATERIAL AND METHODS}

After the Institutional Ethics Committee of Krishna Institute Medical Sciences Deemed university , KARAD, approved the study protocol and written informed consent from the patients was obtained .240 Patient were selected and randomized by computer .60 patient were selected for each one of four group.30 male and 30 females patient were allocated to each group. Variation in perfusion index was studied separately in four technique of anesthesia namely neuraxial anesthesia, inhalational anesthesia,total intravenous anesthesia and general anesthesia with endotracheal intubation .

All patient were given 10 diazepam 12 hours before operation

All patients were adequately hydrated with $500 \mathrm{ml}$ of colloid 30 minutes before operation.

\section{INCLUSION CRITERIA FOR THE PATIENTS}

- ASA 1 and ASA 2 patients

- All kind of operations (routine and emergencies)

- Age group 20-40 years

- All type of surgical procedures 


\section{Exclusion criteria for the patients}

- $>40$ years of age

- Pre existing heart disease

- Pre existing renal disease

- Peripheral vascular disease

- Hypertension

- Hypovolumia

- Diabetes mellitus

- Use of vasopressor perioperatively

- PI was monitored with using GE ohmeda Tru Sat Tm model : GTM21089-1512-T3 .Upper and lower limits of P.I reported by the manufacturer is $.02 \%-20 \%$. The oximeter probe was attached to the middle finger of the patient's contra-lateral side of the B.P. monitoring. Finger was wrapped in a towel to minimise heat loss and contamination with ambience light. Simultaneous measurement of electrocardiographic H.R. and non invasive oscillometric arterial blood pressure was measured. $\mathrm{SPO}_{2}$ was also measured.

- Fluid administration was standardised to $10 \mathrm{ml} / \mathrm{kg} /$ minutes of ringer lactacte solutionAmbient temperature was maintained at 25- 26 degree centigrade.

- Each patient was monitored 10 minutes before start of anesthesia and mean basal heart rate mean basal mean arterial pressure and mean basal perfusion index was calculated after observing the parameter for over five minutes.

- Also average change in perfusion index, average change in heart rate and average change of mean arterial pressure was calculated by observing these parameter for 5 minutes by following formula. Maximum value-minimum value/2.

- Perfusion index, heart rate and mean arterial pressure was noted at time of induction and then 2 minutes ,5 minutes 10 minutes and then every 5 minutes.

\section{Significant criterion were set as following}

- Change in Heart rate was taken significant if there was change in heart rate more than 10 beats per minutes.

- Change inMean arterial pressure was taken significant if there was change in MAP was more than $10 \mathrm{~mm}$ of hg.

- Change in perfusion index was taken significant when change in perfusion index was more than $20 \%$

- All three parameters were studied in neuraxial anesthesia, inhalatonal anesthesia, total intravenous anesthesia, and general anesthesia at the time of induction, 2 minutes, and 5 minutes 10 minutes.

- Preanaesthetic check up

- History

Previous Exposure to Anaesthesia

- Medication and Treatment History

- Allergies

- Dentition

- The Airway

- Air way assessment 


\begin{tabular}{|c|c|c|c|}
\hline & $\begin{array}{l}\text { Step 1: Is there evidence of airway } \\
\text { obstruction now? }\end{array}$ & $\begin{array}{l}\text { Step 2: Is there a risk } \\
\text { of anticipated airway } \\
\text { obstruction? }\end{array}$ & $\begin{array}{l}\text { Step 3: Is there a risk of } \\
\text { Aspiration from failure to } \\
\text { PROTECT their airway? }\end{array}$ \\
\hline $\begin{array}{l}\text { Signs \& } \\
\text { Symptoms }\end{array}$ & $\begin{array}{l}\text { Complete Obstruction } \\
\text { Silence without chest rise Or See- } \\
\text { Saw Chest movement Partial } \\
\text { Obstruction } \\
\text { Stridor airway } \\
\text { swelling/compression by hematoma } \\
\text { Secretions - saliva, blood } \\
\text { Snoring - tongue relaxation } \\
\text { Smash - risk of teeth/blood in the } \\
\text { airway }\end{array}$ & $\begin{array}{l}\text { Singe or Sputum } \\
\text { (carbonaceous) - risk } \\
\text { of delayed airway } \\
\text { swelling } \\
\text { inhalational burnStab } \\
\text { or Swelling neck - } \\
\text { risk of delayed } \\
\text { airway compression } \\
\text { from expanding } \\
\text { hematoma or neck } \\
\text { mass }\end{array}$ & Sleepy (low GCS) \\
\hline
\end{tabular}

- Cases Identification of the Patient

- Concurrent Illness

\section{METHODS OF SPINAL ANESTHESIA TECHNIQUES}

Patients were given bupivacaine $0.5 \%$ in 1415 space in left lateral decubitus position with 25 gauge quinces spinal needle under aseptic precautions. Level of anesthesia was set at required level. Dose of bupivacain was customized according to the patient weight type of operation.

\section{METHOD OF INHALATION ANESTHESIA}

Inhalational anesthesia implies GA on mask .after giving $1-2$ microgram $/ \mathrm{kg}$ of Fentanyl and $0.5 \mathrm{mg}$ midazolam, patient was induduced with propofol $2 \mathrm{mg} / \mathrm{kg}$ and anesthesia was maintained with isoflurane11.5 macand nitrous oxide and oxygen in 50-50 ratio.

\section{METHODS OF TIVA}

Total intravenous anesthesia after giving $0.5 \mathrm{mg}$ of midazolam and 1 microgram fentanyl $/ \mathrm{kg}$ patient was given propofol $1-2 \mathrm{mg} / \mathrm{kg}$ and ketamine $1 \mathrm{mg} / \mathrm{kg}$ with all monitors recommended by ASA and oxygen at 61/min via Hudson mask.

\section{METHODS OF GA with ETT}

Patient was taken to operation theatre

Monitors were attached as per ASA standards

Midaz $0.5 \mathrm{mg}$ and fentanyl 1-2 microgram $/ \mathrm{kg}$ was administered as sedation

Thiopentone $5 \mathrm{mg} / \mathrm{kg}$ was administered

Loss of eye reflex was checked

Non depolarizing or depolarizing muscle relaxant was given

Fassiculations were checked

Laryngoscopy was done

Appropriate size endotracheal tube was inserted into trachea under direct visualization of cords

Cuff is inflated to appropriate pressure.

Tube was fixed

Non depolarizing muscle relaxant was given after patient comes out of the effect of succinylcholine, in case given.

Sevoflurane kept at 1-1.5 mac with nitrous oxide at 50:50 ratio.

Tidal volume was set at $8-10 \mathrm{ml} / \mathrm{kg}$ with respiratory rate at $12-14$

\section{STATISTICAL ANALYSIS}

- Analysis was performed using the program SSPS version 20 for windows. 
- The 95\% confidence interval for sensitivity, specificity, and predictive values were calculated Wilson score method as described by Newcombe.

- Numerical data were presented as mean \pm standard categorical data as proportions (\%).

- Statistical significance will be determined at 95\%level of confidence (difference will be considered statistically significant if $\mathrm{p}<0.5$ )

- One way ANOVA was used for comparision of mean differences in demographic data, and baseline values of hemodynamic parameter and perfusion index between groups.For comparision of changes in the crieteria over time in different group, data was analysed by mixed designed ANOVA.

- Multiple tukey post hoc test for multiple comparision was then calculated for significant difference in ANOVA for analysis of categorical variables ,

- The chi square test was used when any expected frequency was $<1$ or $20 \%$ of the expected frequency was $\leq 5$.

The measurement was taken by following methods:

- Percentage change in perfusion index=P.I.max-P.I.min/P.I. $\times 100$

- Preinduction average perfusion index index over 5 minutes $=$ PI max- PImin/2

- Preinduction heart rate variability in percentage over 5 minutes $=$ HR $\max -\mathrm{HR} \min / \mathrm{HR} \min \times 100$

- Preinduction average HR over 5 minutes $=\mathrm{HR} \max -\mathrm{HR} \min / 2$

- Preinduction MAP \% variability= MAP max-MAPmin/MAP $\min \times 100$

- Preinduction MAP average value over $5 \mathrm{~min}=\mathrm{MAP} \max -\mathrm{MAPmin} / 2$

- Percentage perfusion index variatioin overall operation PI max =PI min (whole diuration of operation)/ PImin (whole duration of operation) $\times 100$

\section{OBSERVATION AND ANALYSIS}

Table No. 1: Comparison of four groups of anesthesia with age

\begin{tabular}{|c|c|c|c|c|c|c|c|c|c|c|}
\hline$\stackrel{\infty}{\infty}$ & 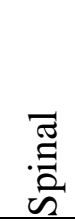 & $0^{\circ}$ & 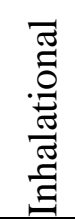 & $\delta^{\circ}$ & $\underset{E}{\mathbb{E}}$ & $\delta^{\circ}$ & 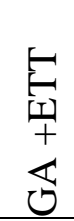 & $\delta^{\circ}$ & $\underset{\tilde{0}}{\tilde{0}}$ & $\delta^{\circ}$ \\
\hline $30 \mathrm{yrs}$ & 3 & 5.00 & 4 & 6.67 & 8 & 13.33 & 7 & 11.67 & 22 & 9.17 \\
\hline $31 \mathrm{yrs}$ & 15 & 25.00 & 17 & 28.33 & 15 & 25.00 & 14 & 23.33 & 61 & 25.42 \\
\hline 32 yrs & 7 & 11.67 & 21 & 35.00 & 12 & 20.00 & 11 & 18.33 & 51 & 21.25 \\
\hline 33 yrs & 15 & 25.00 & 8 & 13.33 & 12 & 20.00 & 14 & 23.33 & 49 & 20.42 \\
\hline 34 yrs & 8 & 13.33 & 10 & 16.67 & 7 & 11.67 & 6 & 10.00 & 31 & 12.92 \\
\hline $35 \mathrm{yrs}$ & 12 & 20.00 & 0 & 0.00 & 6 & 10.00 & 8 & 13.33 & 26 & 10.83 \\
\hline Total & 60 & 100.00 & 60 & 100.00 & 60 & 100.00 & 60 & 100.00 & 240 & 100.00 \\
\hline
\end{tabular}

$* \mathrm{p}<0.05$

Note: There was no significant difference regarding age of patients groups in neuraxial anaesthesia, TIVA, inhalatioal anaesthesia and general anaaesthesia with ETT. 


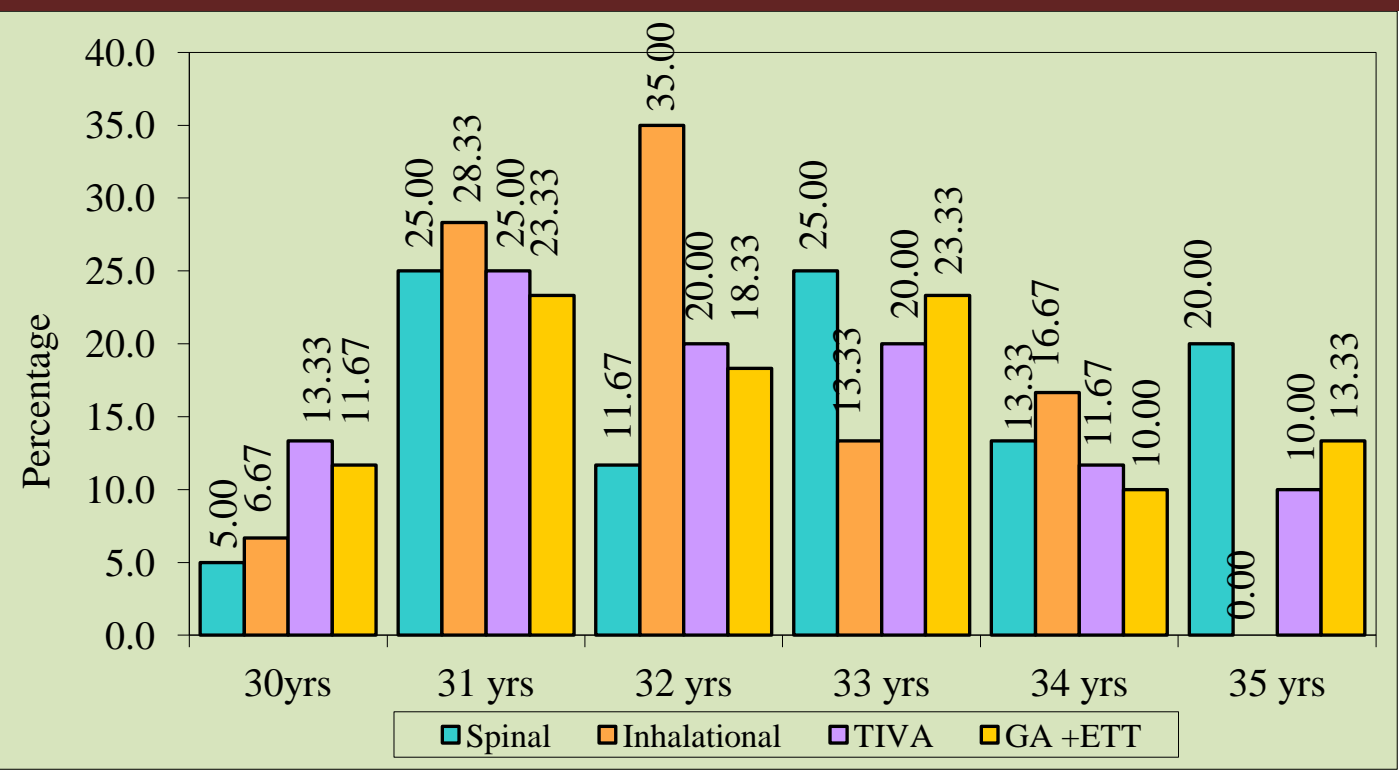

Figure No. 20: Comparison of four groups of anesthesia with age

Table No. 2: Comparison of four groups of anesthesia with ASA status

\begin{tabular}{|c|c|c|c|c|c|c|c|c|c|c|}
\hline$\underset{\longleftarrow}{4}$ & $\begin{array}{l}\bar{\pi} \\
\stackrel{\Xi}{0} \\
\tilde{n}\end{array}$ & $\delta^{\circ}$ & 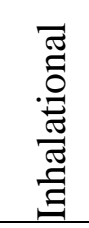 & $\delta^{\circ}$ & $\underset{E}{\gtrless}$ & $\delta^{\circ}$ & $\begin{array}{l}E \\
\qquad \\
+ \\
\mathbb{U}\end{array}$ & $a^{2}$ & 吾 & $\delta^{\circ}$ \\
\hline Score 1 & 33 & 55.00 & 21 & 35.00 & 26 & 43.33 & 20 & 33.33 & 100 & 41.67 \\
\hline Score 2 & 27 & 45.00 & 39 & 65.00 & 34 & 56.67 & 40 & 66.67 & 140 & 58.33 \\
\hline Total & 60 & 100.00 & 60 & 100.00 & 60 & 100.00 & 60 & 100.00 & 240 & 100. \\
\hline
\end{tabular}

Note: Threre was no significant difference between different patient groups regarding ASA status.

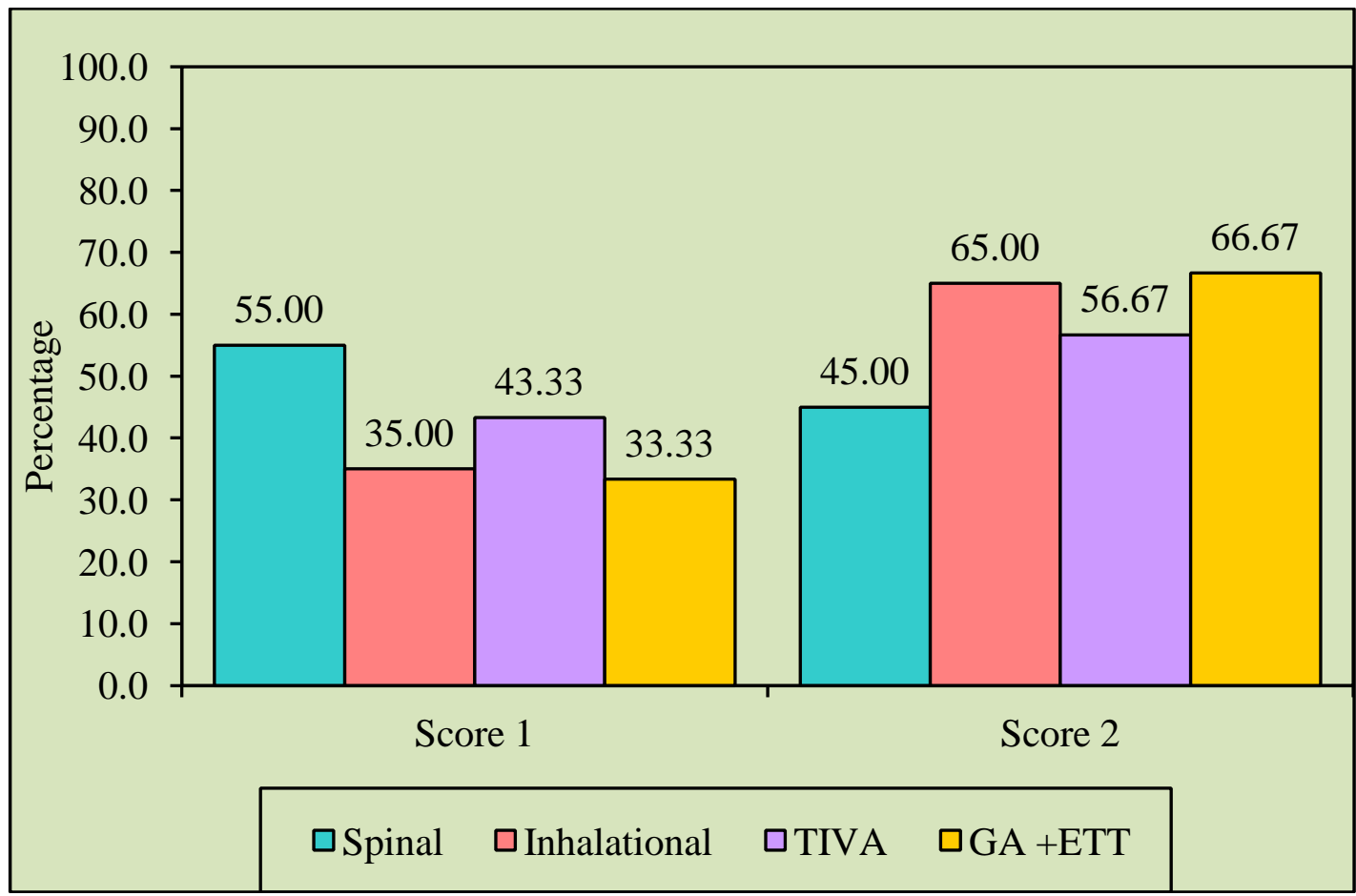

Figure No. 21: Comparison of four groups of anesthesia with ASA status 
Table No. 3: Comparison of four groups of anesthesia with duration of surgery by one way ANOVA

\begin{tabular}{|l|l|l|l|}
\hline Groups & Mean & SD & SE \\
\hline Spinal & 55.40 & 7.56 & 0.98 \\
\hline Inhalational & 30.63 & 6.20 & 0.80 \\
\hline TIVA & 33.18 & 10.30 & 1.33 \\
\hline GA +ETT & 67.48 & 14.81 & 1.91 \\
\hline F-value & 180.2809 & \\
\hline P-value & $0.0001^{*}$ & \\
\hline Pair wise comparisons by Tukeys multiple posthoc procedures & \\
\hline Spinal vs Inhalational & $\mathrm{p}=0.0001^{*}$ & \\
\hline Spinal vs TIVA & $\mathrm{p}=0.0001^{*}$ & \\
\hline Spinal vs GA +ETT & $\mathrm{p}=0.0001^{*}$ & \\
\hline Inhalational vs TIVA & $\mathrm{p}=0.5236$ & \\
\hline Inhalational vs GA +ETT & $\mathrm{p}=0.0001^{*}$ & \\
\hline TIVA vs GA +ETT & $\mathrm{p}=0.0001^{*}$ & \\
\hline
\end{tabular}

*p<0.05

All operation considred in the study was confined to 1-1.5 hours

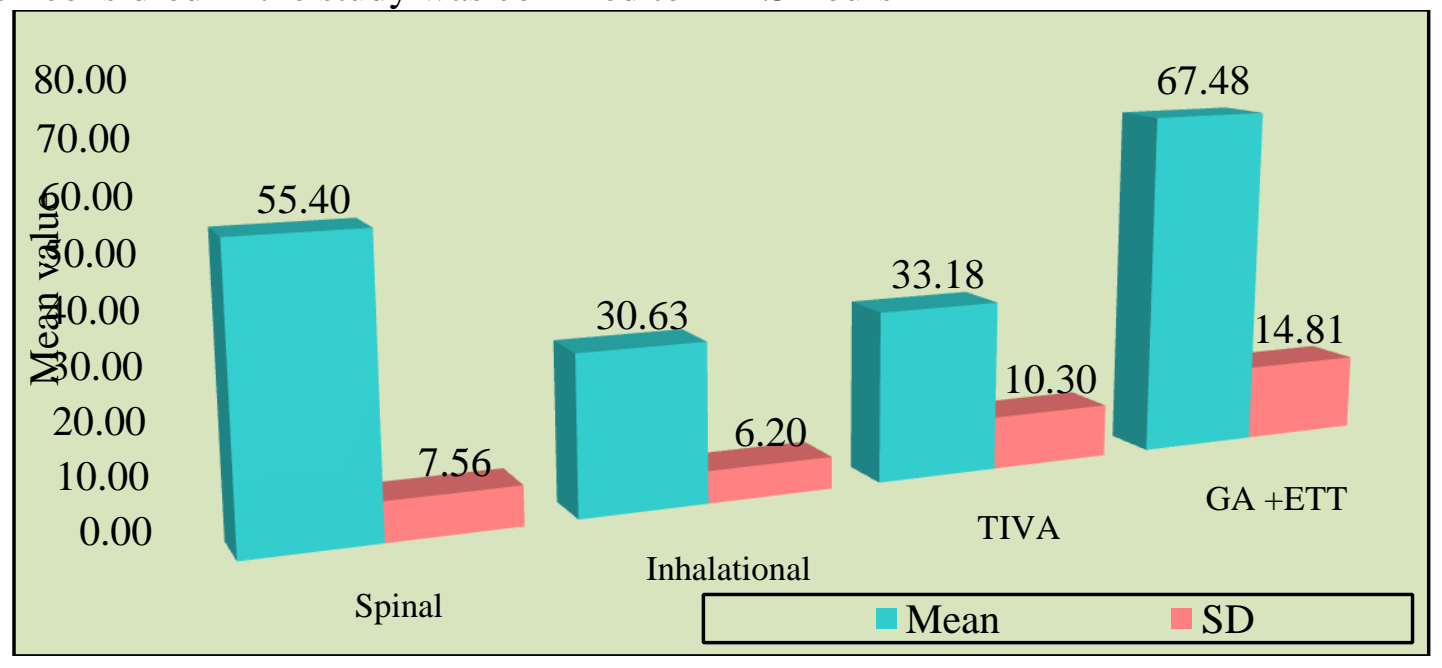

Figure No.22: Comparison of four groups of anesthesia with mean duration of surgery by one way ANOVA 
Table No. 4: Comparison of four groups of anesthesia with weight scores by one way ANOVA

\begin{tabular}{|l|l|l|l|}
\hline Groups & Mean weight in K.g & SD & SE \\
\hline Spinal & 57.42 & 7.06 & 0.91 \\
\hline Inhalational & 58.58 & 9.78 & 1.26 \\
\hline TIVA & 44.02 & 19.76 & 2.55 \\
\hline GA +ETT & 58.02 & 6.28 & 0.81 \\
\hline F-value & 20.5047 & \\
\hline P-value & $0.0001 *$ & \\
\hline Pair wise comparisons by Tukeys multiple posthoc procedures \\
\hline Spinal vs Inhalational & $\mathrm{p}=0.9511$ & \\
\hline Spinal vs TIVA & $\mathrm{p}=0.0001 *$ \\
\hline Spinal vs GA +ETT & $\mathrm{p}=0.9928$ & \\
\hline Inhalational vs TIVA & $\mathrm{p}=0.0001 *$ \\
\hline Inhalational vs GA +ETT & $\mathrm{p}=0.9939$ & \\
\hline TIVA vs GA +ETT & $\mathrm{p}=0.0001 *$ \\
*p $<0.0$ &
\end{tabular}

Note: There was no significant difference between all groups patient population regarding weight

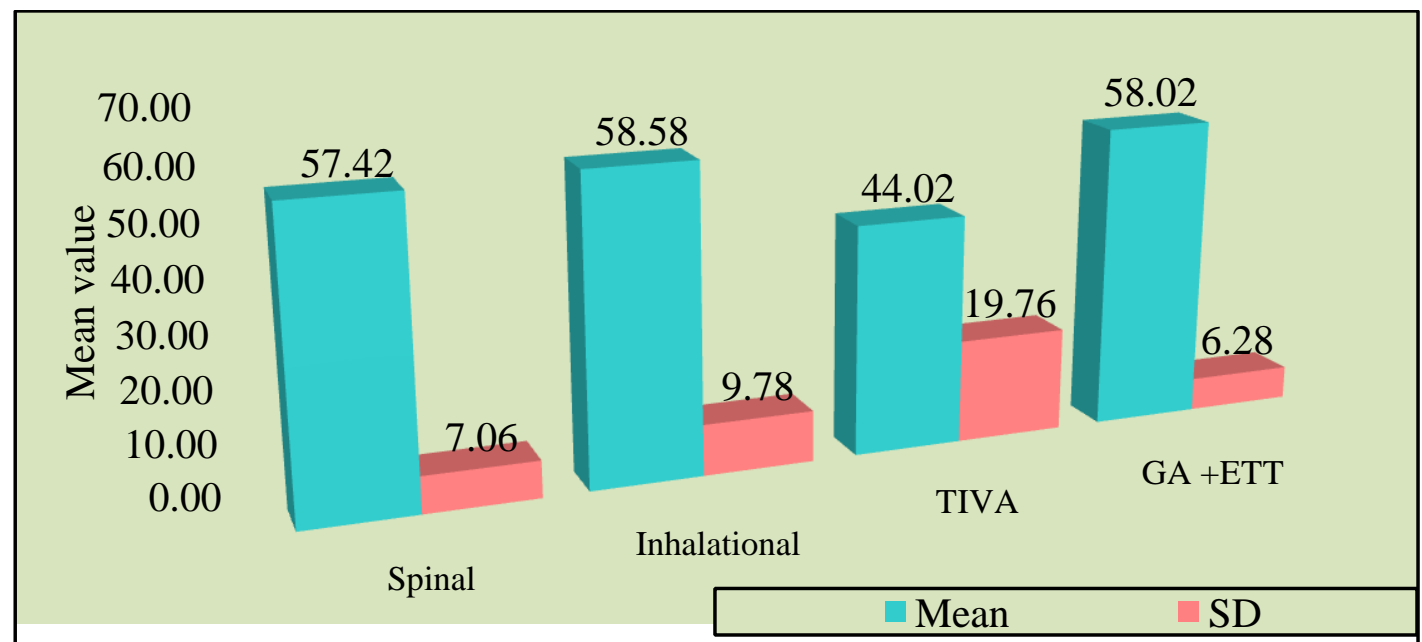

Figure No. 23: Comparison of four groups of anesthesia with weight scores by one way ANOVA 
Table No. 5: Comparison of four groups of anesthesia with PI scores at different time points by one way ANOVA

\begin{tabular}{|c|c|c|c|c|c|c|c|c|}
\hline$\frac{\frac{\tilde{O}}{\pi}}{\frac{\vec{\pi}}{\vec{\sigma}}}$ & 芯 & $\begin{array}{l}\overline{\mathfrak{z}} \\
\stackrel{\mathscr{2}}{n}\end{array}$ & 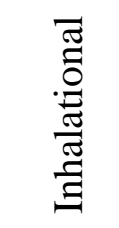 & $\underset{\Downarrow}{\gtrsim}$ & 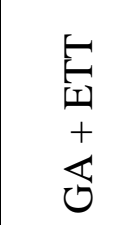 & $\stackrel{\pi}{0}$ & 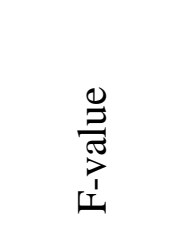 & 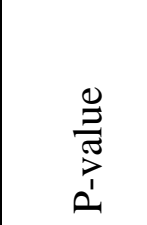 \\
\hline \multirow{2}{*}{$\begin{array}{l}\% \text { change in Perfusion } \\
\text { Index }\end{array}$} & Mean & 8.18 & 11.32 & 12.07 & 9.67 & 10.31 & \multirow[t]{2}{*}{6.7686} & \multirow[t]{2}{*}{$0.0002 *$} \\
\hline & SD & 3.37 & 5.50 & 6.81 & 4.32 & 5.35 & & \\
\hline \multirow{2}{*}{$\begin{array}{ll}\text { Absolute } & \text { baseline } \\
\text { Perfusion Index } & \end{array}$} & Mean & 1.88 & 2.02 & 1.77 & 1.73 & 1.85 & \multirow[t]{2}{*}{1.5174} & \multirow[t]{2}{*}{0.2107} \\
\hline & SD & 0.67 & 0.85 & 0.89 & 0.81 & 0.81 & & \\
\hline \multirow{2}{*}{ Perfusion Index $2 \mathrm{mn}$} & Mean & 2.26 & 2.61 & 2.51 & 1.17 & 2.14 & \multirow[t]{2}{*}{42.8182} & \multirow[t]{2}{*}{$0.0001 *$} \\
\hline & SD & 0.67 & 0.80 & 1.01 & 0.60 & 0.97 & & \\
\hline \multirow{2}{*}{$\begin{array}{l}\% \text { change of Perfusion } \\
\text { Index at } 2 \mathrm{~min}\end{array}$} & Mean & 23.26 & 40.69 & 66.21 & -58.79 & 17.84 & \multirow[t]{2}{*}{82.9493} & \multirow[t]{2}{*}{$0.0001 *$} \\
\hline & SD & 13.92 & 45.15 & 70.03 & 36.27 & 65.47 & & \\
\hline \multirow{2}{*}{ Perfusion Index at $5 \mathrm{mn}$} & Mean & 2.62 & 2.89 & 2.88 & 2.05 & 2.61 & \multirow[t]{2}{*}{11.5158} & \multirow[t]{2}{*}{$0.0001 *$} \\
\hline & $\mathrm{SD}$ & 0.72 & 0.80 & 1.05 & 0.99 & 0.96 & & \\
\hline \multirow{2}{*}{$\begin{array}{l}\% \text { change in Perfusion } \\
\text { Index at } 5 \mathrm{~min}\end{array}$} & Mean & 46.03 & 59.31 & 96.17 & 81.88 & 70.85 & \multirow[t]{2}{*}{0.6279} & \multirow[t]{2}{*}{0.5976} \\
\hline & SD & 25.52 & 50.75 & 81.31 & 427.55 & 218.94 & & \\
\hline \multirow{2}{*}{$\begin{array}{l}\text { Change in Perfusion Index } \\
\text { after } 15 \text { Min of Induction }\end{array}$} & Mean & 170.17 & $\begin{array}{l}178.3 \\
3\end{array}$ & $\begin{array}{l}168.5 \\
5\end{array}$ & 58.47 & 143.88 & \multirow[t]{2}{*}{139.0848} & \multirow[t]{2}{*}{$0.0001 *$} \\
\hline & SD & 52.95 & 30.27 & 36.43 & 24.07 & 62.01 & & \\
\hline \multirow{2}{*}{$\begin{array}{l}\% \text { Change in PI thorough } \\
\text { out operation }\end{array}$} & Mean & 222.02 & $\begin{array}{l}220.9 \\
0\end{array}$ & $\begin{array}{l}213.5 \\
7\end{array}$ & 108.39 & 191.22 & \multirow[t]{2}{*}{109.7802} & \multirow[t]{2}{*}{$0.0001 *$} \\
\hline & SD & 47.23 & 43.26 & 32.31 & 39.38 & 62.93 & & \\
\hline
\end{tabular}

$* \mathrm{p}<0.05$

Note: Change in perfusion index is significant when compared in all four patient groups namely neuraxial,TIVA,inhalational and general anaesthesia with ETT regarding absolute value as well as percentage change except at 5 minutes where these changes are statistically not significant it may be because it the time when patient with GA with ETT group start recovering from surgical stress response of direct laryngoscopy and endotracheal intubation and perfusion index starts increasing due sympatholytic effect of general anaesthesia. 
Table No. 6: Pair wise comparison of four groups of anesthesia with PI scores at different time points by

Tukeys multiple posthoc procedures

\begin{tabular}{|c|c|c|c|c|c|c|}
\hline Variables & $\begin{array}{l}\text { Spinal vs } \\
\text { Inhalationa } \\
1\end{array}$ & $\begin{array}{l}\text { Spinal vs } \\
\text { TIVA }\end{array}$ & $\begin{array}{l}\text { Spinal vs } \\
\text { GA +ETT }\end{array}$ & $\begin{array}{l}\text { Inhalationa } \\
1 \text { vs TIVA }\end{array}$ & $\begin{array}{l}\text { Inhalationa } \\
1 \text { vs GA } \\
+ \text { ETT }\end{array}$ & $\begin{array}{l}\text { TIVA vs } \\
\text { GA +ETT }\end{array}$ \\
\hline $\begin{array}{l}\% \quad \text { change in } \\
\text { Perfusion Index }\end{array}$ & $\mathrm{p}=0.0050^{*}$ & $\begin{array}{l}\mathrm{p}=0.0002 \\
*\end{array}$ & $\mathrm{p}=0.3945$ & $\mathrm{p}=0.8567$ & $\mathrm{p}=0.2983$ & $\mathrm{p}=0.0534$ \\
\hline $\begin{array}{l}\text { ABS BS Perfusion } \\
\text { Index }\end{array}$ & $\mathrm{p}=0.7880$ & $\mathrm{p}=0.8666$ & $\mathrm{p}=0.7538$ & $\mathrm{p}=0.3212$ & $\mathrm{p}=0.2180$ & $p=0.9963$ \\
\hline Perfusion Index $2 \mathrm{mn}$ & $\mathrm{p}=0.0705$ & $\mathrm{p}=0.2822$ & $\begin{array}{l}\mathrm{p}=0.0001 \\
*\end{array}$ & $\mathrm{p}=0.9142$ & $\mathrm{p}=0.0001 *$ & $\begin{array}{l}\mathrm{p}=0.0001 \\
*\end{array}$ \\
\hline $\begin{array}{l}\% \text { change of } \\
\text { Perfusion Index at } 2 \\
\text { min }\end{array}$ & $\mathrm{p}=0.1608$ & $\begin{array}{l}\mathrm{p}=0.0001 \\
*\end{array}$ & $\begin{array}{l}\mathrm{p}=0.0001 \\
*\end{array}$ & $\mathrm{p}=0.0126^{*}$ & $\mathrm{p}=0.0001 *$ & $\begin{array}{l}\mathrm{p}=0.0001 \\
*\end{array}$ \\
\hline $\begin{array}{l}\text { Perfusion Index at } \\
5 \mathrm{mn}\end{array}$ & $\mathrm{p}=0.3374$ & $p=0.3676$ & $\begin{array}{l}\mathrm{p}=0.0032 \\
*\end{array}$ & $\mathrm{p}=0.9999$ & $\mathrm{p}=0.0001 *$ & $\begin{array}{l}\mathrm{p}=0.0001 \\
*\end{array}$ \\
\hline $\begin{array}{l}\% \quad \text { change in } \\
\text { Perfusion Index at } 5 \\
\text { min }\end{array}$ & $\mathrm{p}=0.9874$ & $\mathrm{p}=0.5940$ & $\mathrm{p}=0.8076$ & $\mathrm{p}=0.7942$ & $\mathrm{p}=0.9429$ & $\mathrm{p}=0.9845$ \\
\hline $\begin{array}{l}\text { Change in Perfusion } \\
\text { Index after } 15 \text { Min of } \\
\text { Induction }\end{array}$ & $\mathrm{p}=0.6315$ & $\mathrm{p}=0.9954$ & $\begin{array}{l}\mathrm{p}=0.0001 \\
*\end{array}$ & $\mathrm{p}=0.4813$ & $\mathrm{p}=0.0001 *$ & $\begin{array}{l}\mathrm{p}=0.0001 \\
*\end{array}$ \\
\hline $\begin{array}{l}\text { \%Change in } \begin{array}{l}\text { PI } \\
\text { thorough } \\
\text { operation }\end{array} \\
\end{array}$ & $\mathrm{p}=0.0001^{*}$ & $p=0.6702$ & $\begin{array}{l}\mathrm{p}=0.0001 \\
*\end{array}$ & $\mathrm{p}=0.7599$ & $\mathrm{p}=0.0001 *$ & $\begin{array}{l}\mathrm{p}=0.0001 \\
*\end{array}$ \\
\hline
\end{tabular}

$* \mathrm{p}<0.05$

Note: percentage change of perfusion index after induction of anesthesia is highly significant in all four groups at 2 minutes of anaesthesia when GA with ETT patient group was compared to other three groups because at this duration perfusion index is decreasing due to pressor stress response to direct larygoscopy where as perfusion index is increasing due to sympatholytic response of anaesthesia. 


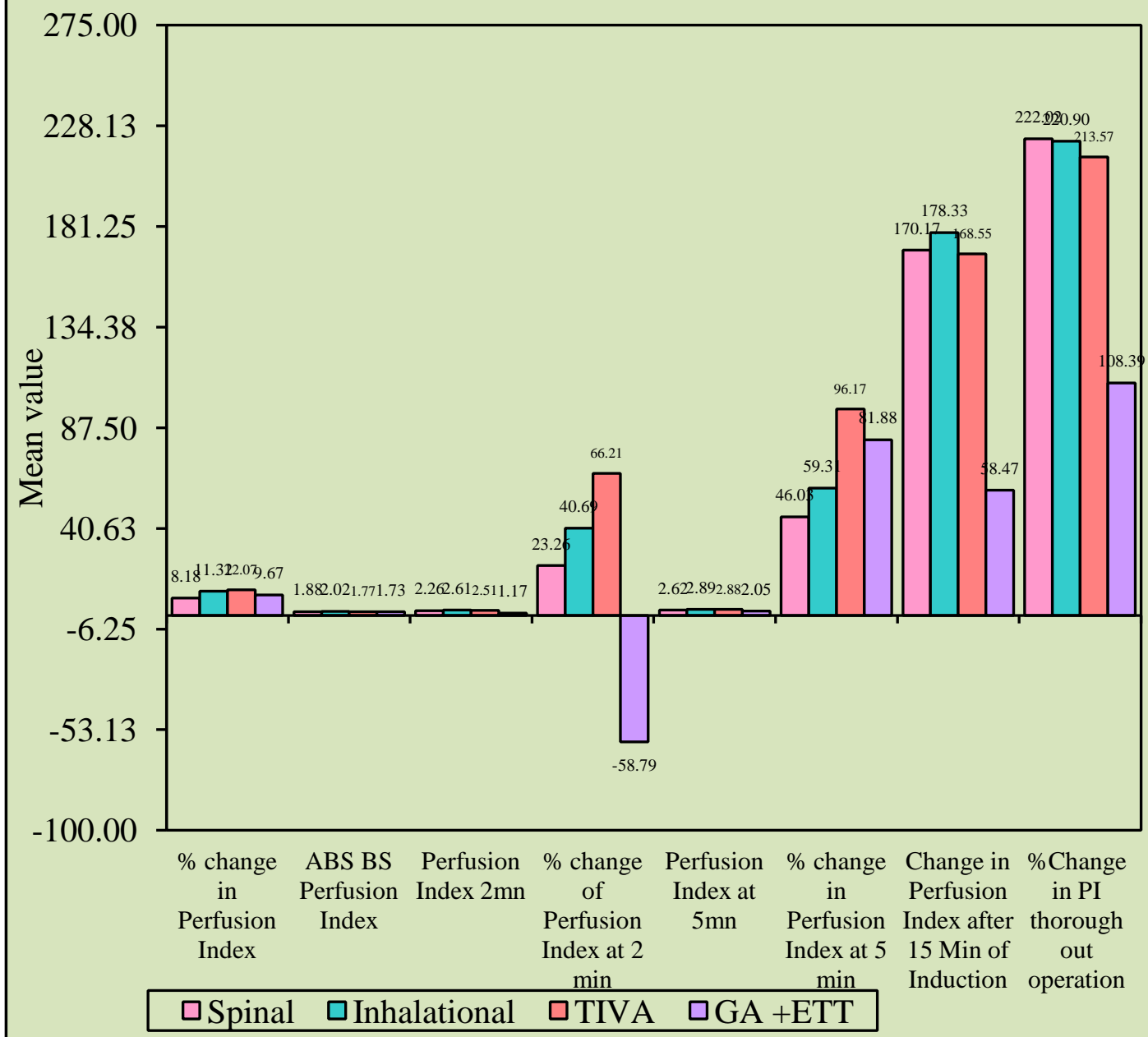

Figure No. 24: Comparison of four groups of anesthesia with PI scores at different time points

Table No. 7: Comparison of four groups of anesthesia with HR scores at different time points

\begin{tabular}{|c|c|c|c|c|c|c|c|c|}
\hline$\frac{\frac{\pi}{0}}{\frac{\pi}{\pi}}$ & 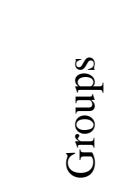 & $\begin{array}{l}\text { ज्ञ } \\
\text { के } \\
\text { के }\end{array}$ & 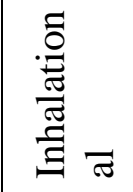 & $\underset{1}{\gtrsim}$ & 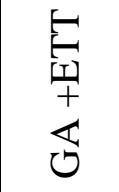 & సేّ & 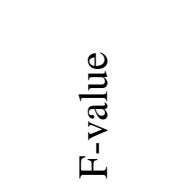 & 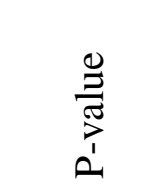 \\
\hline \multirow{2}{*}{$\%$ change in Heart rate } & Mean & 9.22 & 9.22 & 9.33 & 13.10 & 10.22 & \multirow[t]{2}{*}{8.4311} & \multirow[t]{2}{*}{$0.0001 *$} \\
\hline & SD & 3.59 & 4.95 & 4.16 & 7.08 & 5.36 & & \\
\hline \multirow{2}{*}{$\begin{array}{l}\text { Absolute } \\
\text { baselineHeart rate }\end{array}$} & Mean & 82.70 & 76.27 & 79.10 & 79.02 & 79.27 & \multirow[t]{2}{*}{5.6652} & \multirow[t]{2}{*}{$0.0009 *$} \\
\hline & SD & 10.53 & 7.81 & 8.45 & 7.18 & 8.83 & & \\
\hline \multirow{2}{*}{ Heart rate $2 \mathrm{mn}$} & Mean & 72.40 & 66.85 & 71.25 & 96.25 & 76.69 & \multirow[t]{2}{*}{120.4442} & \multirow[t]{2}{*}{$0.0001 *$} \\
\hline & SD & 11.32 & 7.52 & 8.22 & 9.90 & 14.79 & & \\
\hline \multirow{2}{*}{$\begin{array}{l}\% \text { change of Heart rate } \\
\text { at } 2 \text { min }\end{array}$} & Mean & -11.73 & -10.30 & -7.55 & 18.40 & -2.80 & \multirow[t]{2}{*}{130.7674} & \multirow[t]{2}{*}{$0.0001^{*}$} \\
\hline & SD & 5.81 & 7.39 & 6.75 & 15.43 & 15.64 & & \\
\hline Heart rate at $5 \mathrm{mn}$ & Mean & 69.17 & 65.48 & 63.15 & 99.25 & 74.26 & 179.6167 & $0.0001 *$ \\
\hline
\end{tabular}




\begin{tabular}{|c|c|c|c|c|c|c|c|c|}
\hline & SD & 11.57 & 7.15 & 7.21 & 11.92 & 17.53 & & \\
\hline \multirow{2}{*}{$\begin{array}{l}\% \text { change in Heart rate } \\
\text { at } 5 \mathrm{~min}\end{array}$} & Mean & -15.08 & -18.77 & -16.17 & 20.40 & -7.40 & \multirow[t]{2}{*}{237.2633} & \multirow[t]{2}{*}{$0.0001^{*}$} \\
\hline & SD & 6.09 & 10.05 & 9.40 & 11.11 & 18.63 & & \\
\hline \multirow{2}{*}{$\begin{array}{l}\text { Change in Heart rate } \\
\text { after 15 Min of } \\
\text { Induction }\end{array}$} & Mean & 19.67 & 19.40 & 17.48 & 35.40 & 22.99 & \multirow[t]{2}{*}{27.1046} & \multirow[t]{2}{*}{$0.0001 *$} \\
\hline & SD & 8.83 & 11.21 & 11.18 & 16.91 & 14.28 & & \\
\hline \multirow{2}{*}{$\begin{array}{l}\% \text { Change in Heart } \\
\text { rate thorough out } \\
\text { operation }\end{array}$} & Mean & 21.25 & 23.82 & 25.18 & 35.85 & 26.53 & \multirow[t]{2}{*}{34.4519} & \multirow[t]{2}{*}{$0.0001^{*}$} \\
\hline & SD & 6.32 & 6.07 & 10.12 & 10.43 & 10.11 & & \\
\hline
\end{tabular}

$* \mathrm{p}<0.05$

Note: Percentage increase in perfusion index at 2 minutes ,5 minutes and 15 minutes of induction is highly significant

Table No.8: Pair wise comparison of four groups of anesthesia with Heart Rate at different time points by Tukeys multiple posthoc procedures

Note :After induction of anaesthesia pair wise comparision heart rate of different group with GAwithETT is significant because in GA with ETT heart rate increase due to surgical stress where as heart rate decrease in other groups due to sympatholysis

\begin{tabular}{|c|c|c|c|c|c|c|}
\hline Variables & $\begin{array}{l}\text { Spinal vs } \\
\text { Inhalational }\end{array}$ & Spinal vs TIVA & Spinal vs GA +ETT & $\begin{array}{l}\text { Inhalational } \\
\text { vs TIVA }\end{array}$ & $\begin{array}{l}\text { Inhalational } \\
\text { vs GA } \\
+ \text { ETT }\end{array}$ & $\begin{array}{l}\text { TIVA vs } \\
\text { GA +ETT }\end{array}$ \\
\hline $\begin{array}{l}\% \text { change in Heart } \\
\text { rate }\end{array}$ & $\mathrm{p}=0.9999$ & $\mathrm{p}=0.9994$ & $\mathrm{p}=0.0002 *$ & $\mathrm{p}=0.9993$ & $\mathrm{p}=0.0002 *$ & $\mathrm{p}=0.0003^{*}$ \\
\hline ABS BS Heart rate & $\mathrm{p}=0.0002 *$ & $\mathrm{p}=0.0987$ & $\mathrm{p}=0.0870$ & $\mathrm{p}=0.2696$ & $\mathrm{p}=0.2956$ & $\mathrm{p}=0.9999$ \\
\hline Heart rate $2 \mathrm{mn}$ & $\mathrm{p}=0.0064 *$ & $\mathrm{p}=0.9073$ & $\mathrm{p}=0.0001 *$ & $\mathrm{p}=0.0492 *$ & $\mathrm{p}=0.0001 *$ & $\mathrm{p}=0.0001 *$ \\
\hline $\begin{array}{l}\% \text { change of Heart } \\
\text { rate at } 2 \text { min }\end{array}$ & $\mathrm{p}=0.8479$ & $\mathrm{p}=0.0817$ & $\mathrm{p}=0.0001 *$ & $\mathrm{p}=0.4006$ & $\mathrm{p}=0.0001 *$ & $\mathrm{p}=0.0001^{*}$ \\
\hline Heart rate at $5 \mathrm{mn}$ & $\mathrm{p}=0.1621$ & $\mathrm{p}=0.0040^{*}$ & $\mathrm{p}=0.0001^{*}$ & $\mathrm{p}=0.5546$ & $\mathrm{p}=0.0001^{*}$ & $\mathrm{p}=0.0001^{*}$ \\
\hline $\begin{array}{l}\% \text { change in Heart } \\
\text { rate at } 5 \mathrm{~min}\end{array}$ & $\mathrm{p}=0.1355$ & $\mathrm{p}=0.9210$ & $\mathrm{p}=0.0001 *$ & $\mathrm{p}=0.4239$ & $\mathrm{p}=0.0001 *$ & $\mathrm{p}=0.0001 *$ \\
\hline $\begin{array}{l}\text { Change in Heart rate } \\
\text { after } 15 \text { Min of } \\
\text { Induction }\end{array}$ & $\mathrm{p}=0.9994$ & $\mathrm{p}=0.7696$ & $\mathrm{p}=0.0001 *$ & $\mathrm{p}=0.8320$ & $\mathrm{p}=0.0001 *$ & $\mathrm{p}=0.0001 *$ \\
\hline $\begin{array}{l}\% \text { Change in Heart } \\
\text { rate thorough out } \\
\text { operation }\end{array}$ & $\mathrm{p}=0.0001^{*}$ & $\mathrm{p}=0.0540$ & $\mathrm{p}=0.0001 *$ & $\mathrm{p}=0.8139$ & $\mathrm{p}=0.0001^{*}$ & $\mathrm{p}=0.0001 *$ \\
\hline
\end{tabular}

$* \mathrm{p}<0.05$ 


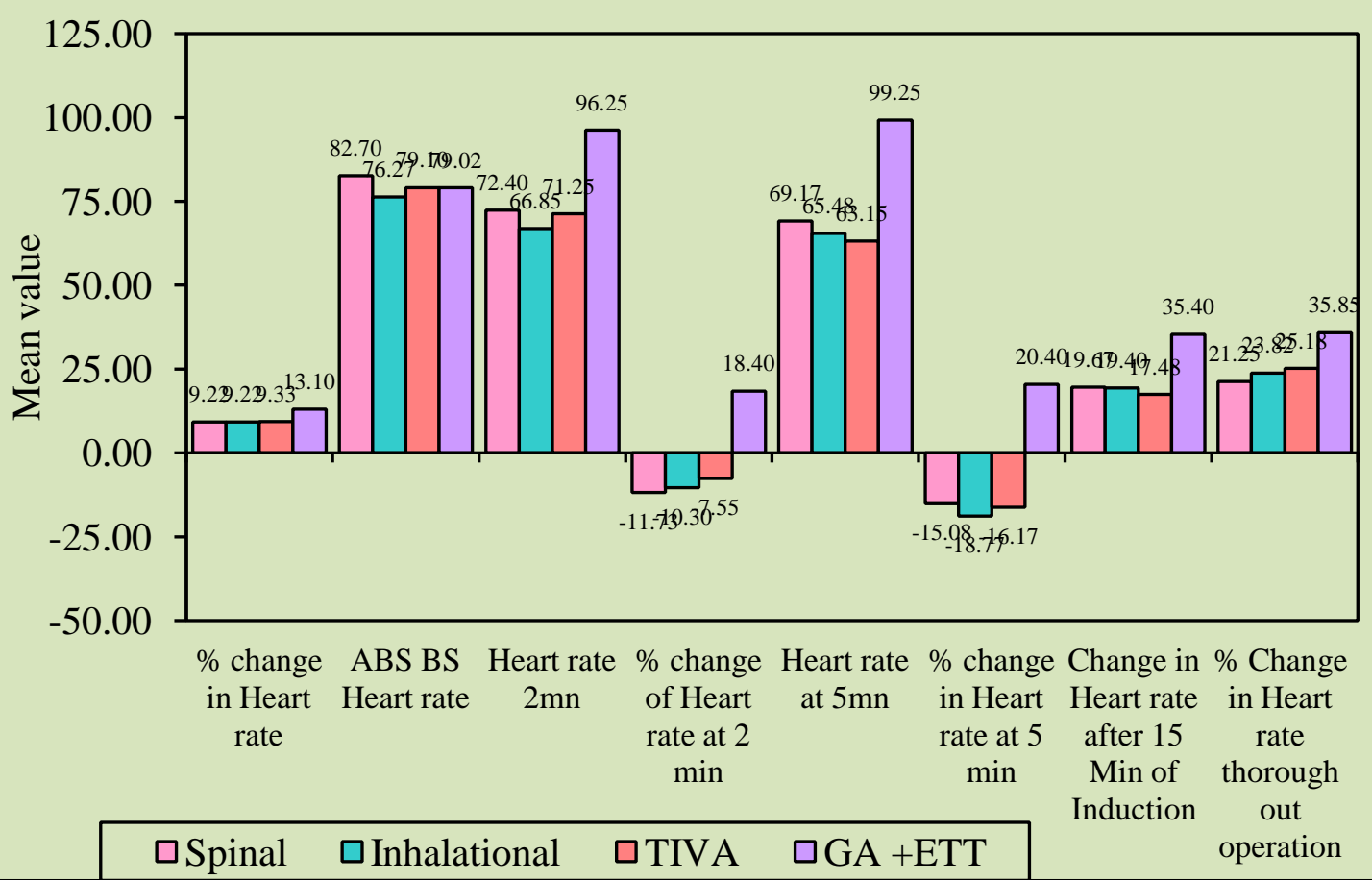

Figure No. 25: Comparison of four groups of anesthesia with heart rate scores at different time points

Table No. 9: Comparison of four groups of anesthesia with MAP scores at different time points Note: MAP percentage change is significant after induction of anaesthesia

\begin{tabular}{|c|c|c|c|c|c|c|c|c|}
\hline$\frac{\frac{\pi}{0}}{\frac{\pi}{\pi}}$ & $\begin{array}{l}\stackrel{0}{O} \\
0 \\
0\end{array}$ & $\begin{array}{l}\bar{\Xi} \\
\stackrel{\Xi}{\tilde{n}} \\
\bar{n}\end{array}$ & 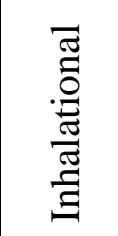 & 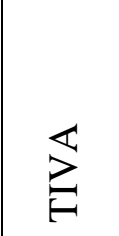 & 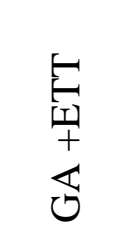 & 䓪 & 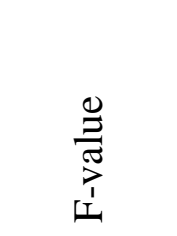 & 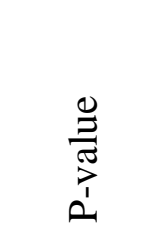 \\
\hline \multirow{2}{*}{ Baseline MAP } & Mean & 11.17 & 8.72 & 9.33 & 9.43 & 9.66 & \multirow[t]{2}{*}{2.5421} & \multirow[t]{2}{*}{0.0570} \\
\hline & SD & 6.25 & 3.77 & 4.96 & 5.14 & 5.16 & & \\
\hline \multirow{2}{*}{$\begin{array}{l}\text { Absolute } \\
\text { baselineMAP }\end{array}$} & Mean & 84.13 & 81.53 & 82.70 & 84.07 & 83.11 & \multirow[t]{2}{*}{3.2080} & \multirow[t]{2}{*}{$0.0238^{*}$} \\
\hline & SD & 6.37 & 6.28 & 4.49 & 3.86 & 5.44 & & \\
\hline \multirow{2}{*}{$\begin{array}{l}\text { \%Change in MAP } \\
15 \text { Min of } \\
\text { Induction }\end{array}$} & Mean & 18.58 & 19.20 & 26.00 & 46.80 & 27.65 & \multirow[t]{2}{*}{88.5010} & \multirow[t]{2}{*}{$0.0001 *$} \\
\hline & SD & 9.81 & 9.81 & 11.36 & 12.30 & 15.75 & & \\
\hline \multirow{2}{*}{$\begin{array}{lr}\text { \%Change } & \text { in } \\
\text { MAPthorough out } \\
\text { operation }\end{array}$} & Mean & 26.52 & 34.30 & 29.22 & 71.76 & 40.32 & \multirow[t]{2}{*}{136.3881} & \multirow[t]{2}{*}{$0.0001 *$} \\
\hline & SD & 8.51 & 11.75 & 8.51 & 22.31 & 22.91 & & \\
\hline
\end{tabular}


Table10: Pair wise comparison of four groups of anesthesia with Heart Rate at different time points by

Tukeys multiple posthoc procedures

\begin{tabular}{|l|l|l|l|l|l|l|}
\hline Variables & $\begin{array}{l}\text { Spinal vs } \\
\text { Inhalational }\end{array}$ & $\begin{array}{l}\text { Spinal vs } \\
\text { TIVA }\end{array}$ & $\begin{array}{l}\text { Spinal vs } \\
\text { GA +ETT }\end{array}$ & $\begin{array}{l}\text { Inhalational } \\
\text { vs TIVA }\end{array}$ & $\begin{array}{l}\text { Inhalational } \\
\text { vs GA +ETT }\end{array}$ & $\begin{array}{l}\text { TIVA vs } \\
\text { GA +ETT }\end{array}$ \\
\hline $\begin{array}{l}\text { Baseline Blood } \\
\text { Pressure }\end{array}$ & $\mathrm{p}=0.0429^{*}$ & $\mathrm{p}=0.2012$ & $\mathrm{p}=0.2463$ & $\mathrm{p}=0.9117$ & $\mathrm{p}=0.8688$ & $\mathrm{p}=0.9996$ \\
\hline ABS BSL BP & $\mathrm{p}=0.0397^{*}$ & $\mathrm{p}=0.4598$ & $\mathrm{p}=0.9999$ & $\mathrm{p}=0.6325$ & $\mathrm{p}=0.0477^{*}$ & $\mathrm{p}=0.5022$ \\
\hline $\begin{array}{l}\text { \% Change in BP } \\
\begin{array}{l}\text { In Min of } \\
\text { Induction }\end{array}\end{array}$ & $\mathrm{p}=0.9896$ & $\mathrm{p}=0.0011^{*}$ & $\mathrm{p}=0.0001^{*}$ & $\mathrm{p}=0.0034^{*}$ & $\mathrm{p}=0.0001^{*}$ & $\mathrm{p}=0.0001^{*}$ \\
\hline $\begin{array}{l}\text { \%Change in } \\
\text { MAP thorough } \\
\text { out operation }\end{array}$ & $\mathrm{p}=0.0118$ & $\mathrm{p}=0.7127$ & $\mathrm{p}=0.0001^{*}$ & $\mathrm{p}=0.1881$ & $\mathrm{p}=0.0001^{*}$ & $\mathrm{p}=0.0001^{*}$ \\
\hline
\end{tabular}

*p $<0.05$

Note: There is significant difference when other techniques are compared with GA with ETT when heart rate is compared.

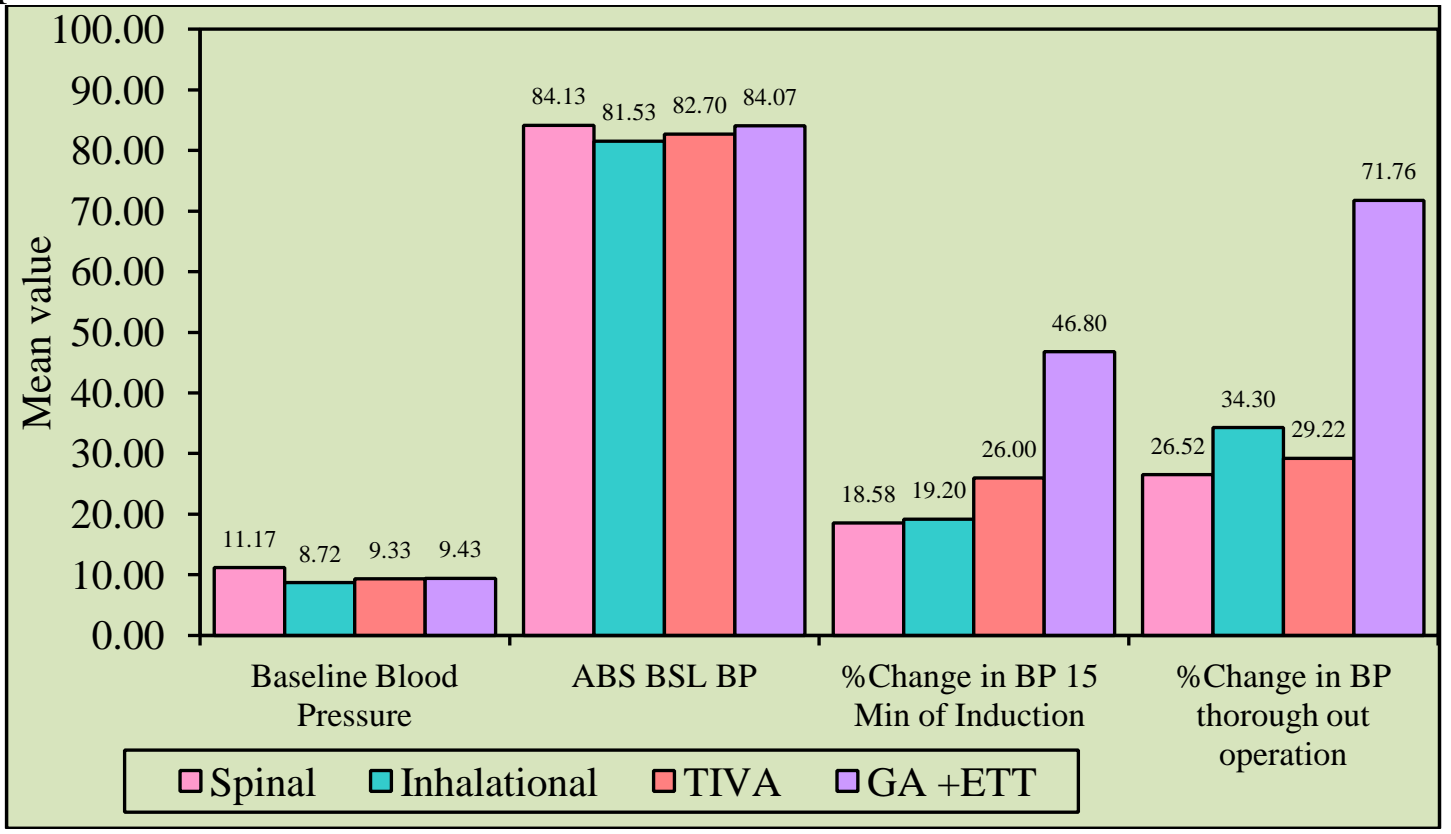

Figure No. 26: Comparison of four groups of anesthesia with BP scores at different time points

Table No. 11: Comparison of four groups of anesthesia with MAP scores at different time point

\begin{tabular}{|c|c|c|c|c|c|c|c|c|}
\hline 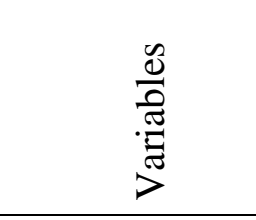 & $\begin{array}{l}0 \\
\tilde{\Xi} \\
\dot{0}\end{array}$ & 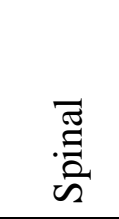 & 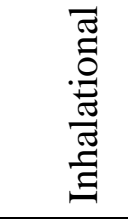 & $\sum_{i}^{\mathbb{Z}}$ & 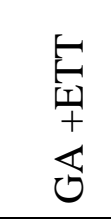 & $\stackrel{\sqrt[5]{0}}{\stackrel{0}{\circ}}$ & 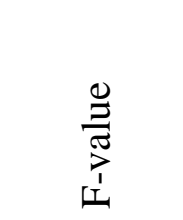 & 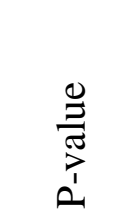 \\
\hline \multirow{2}{*}{ MAP in2 min } & Mean & 79.30 & 76.50 & 72.38 & $\begin{array}{l}108.9 \\
3\end{array}$ & 84.28 & \multirow[t]{2}{*}{180.3524} & \multirow[t]{2}{*}{$\begin{array}{l}0.0001 \\
*\end{array}$} \\
\hline & SD & 6.79 & 7.26 & 9.71 & 13.31 & 17.35 & & \\
\hline
\end{tabular}




\begin{tabular}{|c|c|c|c|c|c|c|c|c|}
\hline \multirow{2}{*}{$\begin{array}{l}\text { Change In MAP } \\
\text { At } 2 \text { min }\end{array}$} & Mean & -5.48 & -5.07 & -10.18 & 22.85 & 0.53 & \multirow[t]{2}{*}{259.7323} & \multirow[t]{2}{*}{$\begin{array}{l}0.0001 \\
*\end{array}$} \\
\hline & SD & 3.36 & 3.90 & 7.87 & 11.01 & 14.92 & & \\
\hline \multirow{2}{*}{$\begin{array}{l}\text { MAP after } \\
5 \mathrm{~min}\end{array}$} & Mean & 75.45 & 71.23 & 66.02 & $\begin{array}{l}102.8 \\
5\end{array}$ & 78.89 & \multirow[t]{2}{*}{161.4081} & \multirow[t]{2}{*}{$\begin{array}{l}0.0001 \\
*\end{array}$} \\
\hline & SD & 7.56 & 8.02 & 10.09 & 13.35 & 17.39 & & \\
\hline \multirow{2}{*}{$\begin{array}{l}\text { Change in MAP } \\
\text { at } 5 \mathrm{~min}\end{array}$} & Mean & -9.38 & -9.50 & -16.28 & 19.60 & -3.89 & \multirow[t]{2}{*}{175.7472} & \multirow[t]{2}{*}{$\begin{array}{l}0.0001 \\
*\end{array}$} \\
\hline & $\mathrm{SD}$ & 5.16 & 5.60 & 8.91 & 14.56 & 16.70 & & \\
\hline
\end{tabular}

$* \mathrm{p}<0.05$

Note: Change in MAP at 2 and 5 minutes are significant at 2 minutes and 5 minutes

Table No. 12: Pair wise comparison of four groups of anesthesia with Heart Rate at different time points by Tukeys multiple posthoc procedures

\begin{tabular}{|l|l|l|l|l|l|l|}
\hline Variables & $\begin{array}{l}\text { Spinal vs } \\
\text { Inhalational }\end{array}$ & $\begin{array}{l}\text { Spinal vs } \\
\text { TIVA }\end{array}$ & $\begin{array}{l}\text { Spinal vs } \\
\text { GA+ETT }\end{array}$ & $\begin{array}{l}\text { Inhalational } \\
\text { vs TIVA }\end{array}$ & $\begin{array}{l}\text { Inhalational } \\
\text { vs } \\
+E T T\end{array}$ & $\begin{array}{l}\text { TIVA } \\
\text { GA +ETT }\end{array}$ \\
\hline MAP 2 min & $\mathrm{p}=0.3820$ & $\mathrm{p}=0.0005^{*}$ & $\mathrm{p}=0.0001^{*}$ & $\mathrm{p}=0.0883$ & $\mathrm{p}=0.0001^{*}$ & $\mathrm{p}=0.0001^{*}$ \\
\hline Change In MAP At 2min & $\mathrm{p}=0.9892$ & $\mathrm{p}=0.0021^{*}$ & $\mathrm{p}=0.0001^{*}$ & $\mathrm{p}=0.0006^{*}$ & $\mathrm{p}=0.0001^{*}$ & $\mathrm{p}=0.0001^{*}$ \\
\hline MAP after 5min & $\mathrm{p}=0.0969$ & $\mathrm{p}=0.0001^{*}$ & $\mathrm{p}=0.0001^{*}$ & $\mathrm{p}=0.0226^{*}$ & $\mathrm{p}=0.0001^{*}$ & $\mathrm{p}=0.0001^{*}$ \\
\hline Change in MAP at 5 min & $\mathrm{p}=0.9999$ & $\mathrm{p}=0.0003^{*}$ & $\mathrm{p}=0.0001^{*}$ & $\mathrm{p}=0.0004^{*}$ & $\mathrm{p}=0.0001^{*}$ & $\mathrm{p}=0.0001^{*}$ \\
\hline
\end{tabular}

${ }^{*} \mathrm{p}<0.05$ At $2 \mathrm{~min}$ and $5 \mathrm{~min}$ absolute value and change in MAP changes significantly

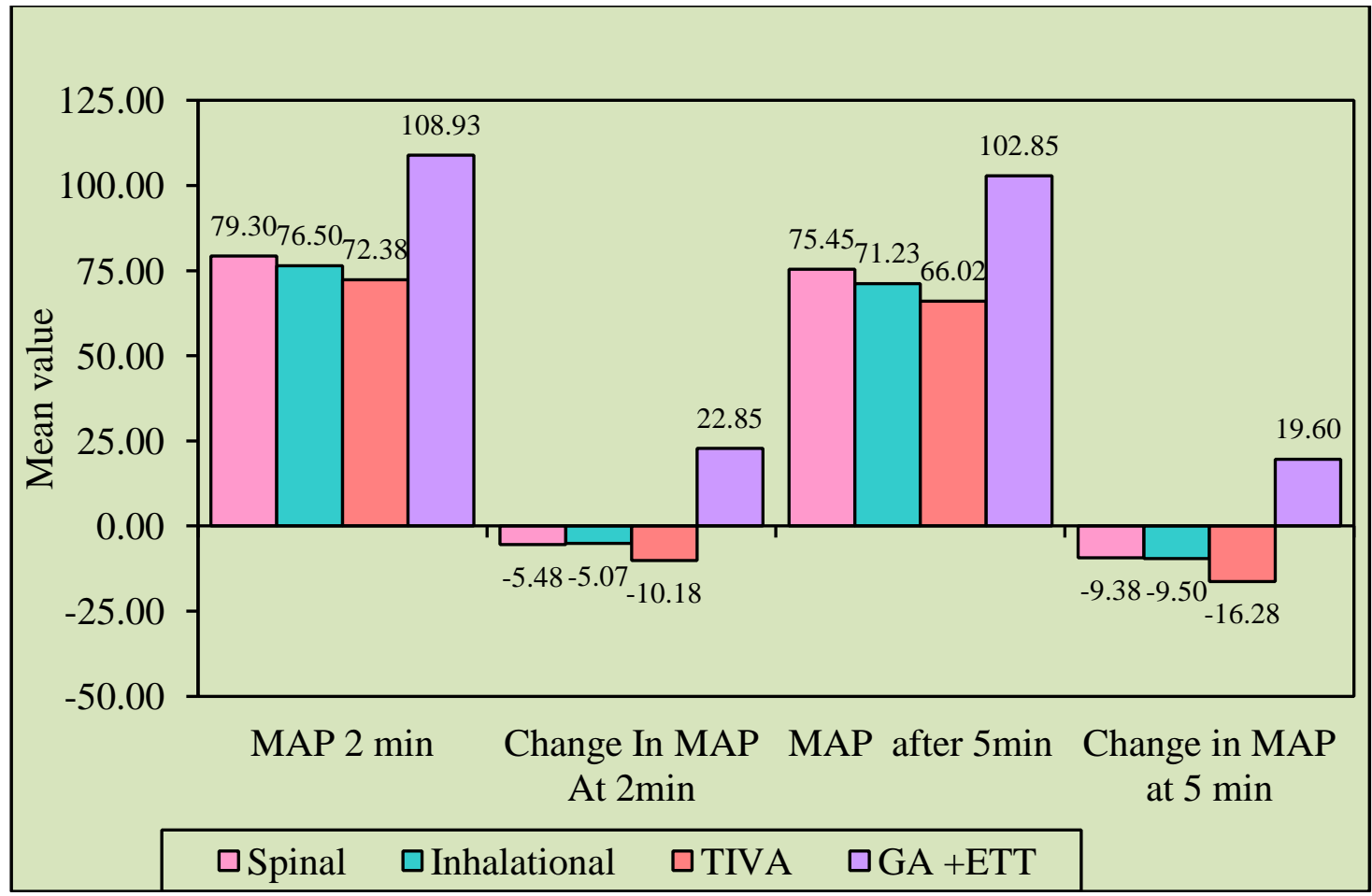

Figure No. 27: Comparison of four groups of anesthesia with MAP scores at different time points 
Table No. 13: Comparison of mean of Perfusion index Heart Rate Mean arterial pressure pre induction and after 2 min and 5 min.

INFERENCE: perfusion index increases at $2 \mathrm{~min}$. and $5 \mathrm{~min}$. In neuraxial, TIVA, inhalational but decreases in GAwith ETT.

\begin{tabular}{|c|c|c|c|c|c|c|c|c|c|c|c|c|}
\hline & \multicolumn{3}{|c|}{$\begin{array}{l}\text { Neuraxial } \\
\text { anesthesia }\end{array}$} & \multicolumn{3}{|c|}{$\begin{array}{l}\text { Inhalational } \\
\text { anesthesia }\end{array}$} & \multicolumn{3}{|c|}{ TIVA } & \multicolumn{3}{|c|}{ GA with ETT } \\
\hline & Basal & $\begin{array}{l}2 \\
\min \end{array}$ & $\begin{array}{l}5 \\
\min \end{array}$ & Basal & $\begin{array}{l}2 \\
\min \end{array}$ & $\begin{array}{l}5 \\
\min \end{array}$ & Basal & $\begin{array}{l}2 \\
\min \end{array}$ & $\begin{array}{l}5 \\
\min \end{array}$ & Basal & $2 \min$ & $5 \mathrm{~min}$ \\
\hline $\begin{array}{l}\text { Perfusion } \\
\text { Index }\end{array}$ & 1.91 & 2.27 & 2.5 & 2.06 & 2.66 & 2.8 & 1.67 & 2.48 & 2.79 & 1.27 & 1.19 & 1.75 \\
\hline Heart Rate & 82.3 & 72.4 & 69.1 & 81.7 & 66.3 & 65.48 & 79.45 & 61.2 & 65.35 & 63.28 & 71.25 & 99.25 \\
\hline $\begin{array}{l}\text { Mean arterial } \\
\text { pressure }\end{array}$ & 82.39 & 79.3 & 75.4 & 97.65 & 76.56 & 71.23 & 92.7 & 72.15 & 66.01 & 87.65 & 108.72 & 102.611 \\
\hline
\end{tabular}

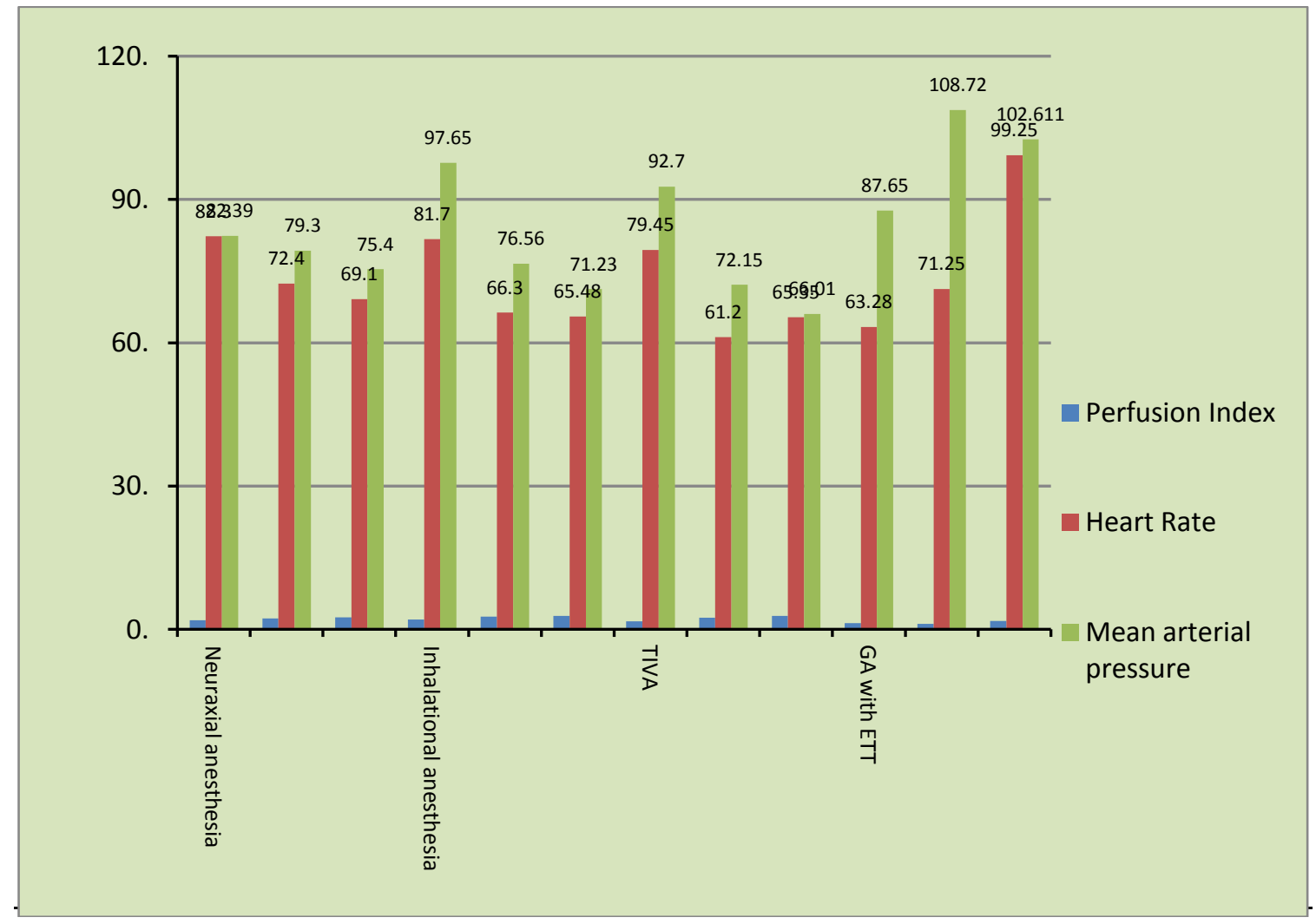

Figur No. 28: Mean of Perfusion Index, Heart Rate, Mean Arterial Pressure Preinduction, 2 Minutes and 5 Minutes

Table No. 14: Trend of perfusion index in a single procedure in all 4 groups of patients

\begin{tabular}{|l|l|l|l|l|}
\hline $\begin{array}{l}\text { Duration of } \\
\text { Operation }\end{array}$ & $\begin{array}{l}\text { GA with } \\
\text { ETT }\end{array}$ & $\begin{array}{l}\text { Neuraxial } \\
\text { anesthesia }\end{array}$ & $\begin{array}{l}\text { Inhalational } \\
\text { anesthesia }\end{array}$ & TIVA \\
\hline 0 & 1.4 & 1.2 & 1.25 & 1.3 \\
\hline 3 & 1.2 & 1.4 & 1.3 & 1.4 \\
\hline 6 & 0.9 & 1.8 & 1.4 & 1.5 \\
\hline 9 & 1.1 & 1.9 & 1.6 & 1.62 \\
\hline 12 & 1.25 & 2.0 & 1.7 & 1.73 \\
\hline 15 & 1.30 & 2.2 & 1.8 & 1.83 \\
\hline
\end{tabular}




\begin{tabular}{|l|l|l|l|l|}
\hline 18 & 1.35 & 2.4 & 1.9 & 1.93 \\
\hline 21 & 1.45 & 2.6 & 1.92 & 1.98 \\
\hline 24 & 165 & 2.8 & 1.95 & 1.90 \\
\hline 27 & 1.7 & 2.9 & 1.96 & 2.00 \\
\hline 30 & 1.8 & 3.2 & 2.00 & 2.02 \\
\hline 33 & 1.9 & 3.4 & 2.2 & 2.04 \\
\hline 36 & 2.1 & 2.8 & 2.22 & 2.05 \\
\hline 39 & 2.2 & 2.9 & 2.3 & 2.08 \\
\hline 42 & 2.5 & 3.0 & 2.32 & 2.1 \\
\hline 45 & 2.8 & 2.9 & 2.35 & 2.2 \\
\hline 48 & 1.8 & 3.1 & 2.6 & 2.6 \\
\hline 51 & 1.5 & 3.2 & 2.7 & 2.7 \\
\hline 54 & 1.3 & 3.1 & 2.7 & 2.9 \\
\hline 57 & 1.2 & 2.9 & 2.6 & 3.7 \\
\hline 60 & 1.4 & 2.98 & 2.7 & 2.8 \\
\hline 63 & 1.6 & 2.78 & 2.9 & 2.9 \\
\hline
\end{tabular}

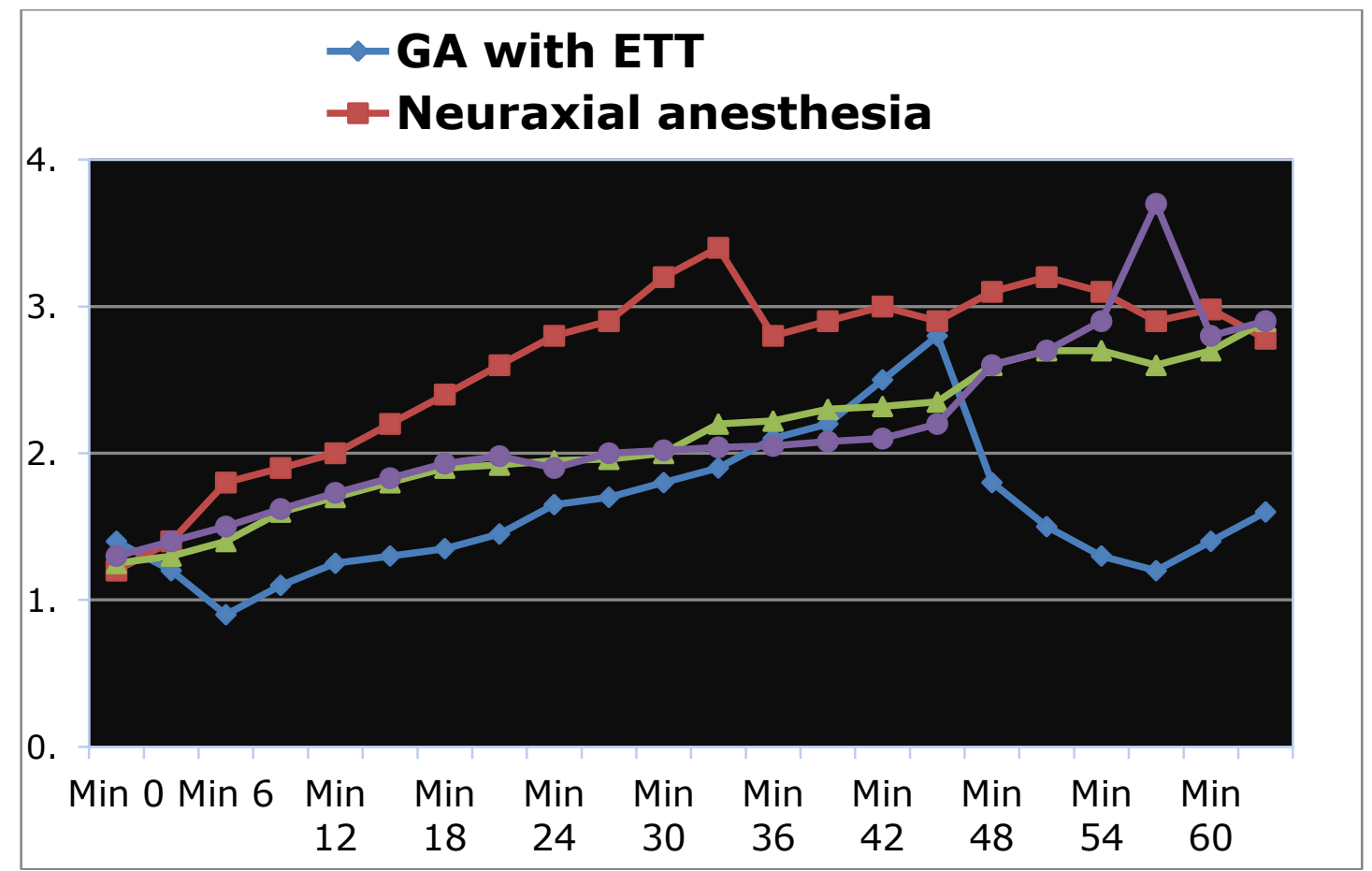

Figure No. 29: Trend of perfusion index in a single procedure in all 4 groups of patients Note: Trend of perfusion index in an indivisual operation . There is an initial decrease in P.I. in GA with ETT group patient then again there is decrease at the end of anaesthesia showing surgical stress response to intubation and extubation respectively.In all other three groups there is consistent increase in the perfusion index showing absence of pressor response and sympathetic stimulation. 
Table No. 15: sensitivity specificity positive predictive value negative predictive value of perfusion index with heart rate as gold standard at 2 minutes for onset of anaesthesia in neuraxial, tiva inhalational group

\begin{tabular}{|l|l|l|l|}
\hline & $\begin{array}{l}\text { Neuraxial } \\
\text { anesthesia }\end{array}$ & Inhalational anesthesia & TIVA \\
\hline Sensitivity & $66 \%$ & $66 \%$ & $58.065 \%$ \\
\hline Specificity & $20.5 \%$ & $37.03 \%$ & $17.24 \%$ \\
\hline Positive Predictive Value & $51.28 \%$ & $54.05 \%$ & $42.85 \%$ \\
\hline Negative predictive value & $38.095 \%$ & $43.47 \%$ & $27.78 \%$ \\
\hline
\end{tabular}

Table No. 16: Sensitivity specificity positive predictive value negative predictive value of perfusion index with heart rate as gold standard at 2 minutes for stress response to intubation in ga with ett

\begin{tabular}{|l|l|}
\hline & GA with ETT \\
\hline Sensitivity & $98.1 \%$ \\
\hline Specificity & $16.67 \%$ \\
\hline Positive Predictive Value & $7.47 \%$ \\
\hline Negative predictive value & $83.33 \%$ \\
\hline
\end{tabular}

Table No. 17: Sensitivity specificity positive predictive value negative predictive value of mean arterial pressure with heart rate as gold standard at 2 minutes for onset of anesthesia.

\begin{tabular}{|l|l|l|l|}
\hline & $\begin{array}{l}\text { Neuraxial } \\
\text { anesthesia }\end{array}$ & Inhalational anesthesia & TIVA \\
\hline Sensitivity & $52 \%$ & $20 \%$ & $59 \%$ \\
\hline Specificity & $46 \%$ & $40 \%$ & $30 \%$ \\
\hline Positive Predictive Value & $71 \%$ & $80 \%$ & $62 \%$ \\
\hline Negative predictive value & $72 \%$ & $84 \%$ & $54 \%$ \\
\hline
\end{tabular}

Table No. 18: Sensitivity specificity positive predictive value negative predictive value of mean arterial pressure with heart rate as gold standard at 2 minutes for stress response to intubation

\begin{tabular}{|l|l|}
\hline & GA with ETT \\
\hline Sensitivity & $92.4 \%$ \\
\hline Specificity & $6 \%$ \\
\hline Positive Predictive Value & $71 \%$ \\
\hline Negative predictive value & $61 \%$ \\
\hline
\end{tabular}

Table No.19: With heart rate as gold standard Perfusion index sensitivity specificity positive predictive value and negative predictive value in neuraxial, tiva, inhalational in 5 mints for onset of anaesthesia

\begin{tabular}{|l|l|l|l|}
\hline & $\begin{array}{l}\text { Neuraxial } \\
\text { anesthesia }\end{array}$ & $\begin{array}{l}\text { Inhalational } \\
\text { anesthesia }\end{array}$ & TIVA \\
\hline Sensitivity & $100 \%$ & $90.566 \%$ & $93.4 \%$ \\
\hline Specificity & $11 \%$ & $16.66 \%$ & $7.14 \%$ \\
\hline Positive Predictive Value & $85 \%$ & $7.47 \%$ & $76.78 \%$ \\
\hline Negative predictive value & $5 \%$ & $83.33 \%$ & $75 \%$ \\
\hline
\end{tabular}


Table No. 20: With heart rate as gold standard Perfusion index sensitivity specificity positive predictive value and negative predictive value in GA with ETT group in 5 mints for stress response to i ntubation

\begin{tabular}{|l|l|}
\hline & GA with ETT \\
\hline Sensitivity & $98.1 \%$ \\
\hline Specificity & $16.67 \%$ \\
\hline Positive Predictive Value & $7.47 \%$ \\
\hline Negative predictive value & $83.33 \%$ \\
\hline
\end{tabular}

Table No. 21: Sensitivity specificity positive predictive value negative predictive value of mean arterial pressure with heart rate as gold standard at 5minutes for onset of anesthesia.

\begin{tabular}{|l|l|l|l|}
\hline & Neuraxial anesthesia & Inhalational anesthesia & TIVA \\
\hline Sensitivity & $59.61 \%$ & $53.7 \%$ & $56 \%$ \\
\hline Specificity & $80.76 \%$ & $83.3 \%$ & $79 \%$ \\
\hline Positive Predictive Value & $91.7 \%$ & $96.6 \%$ & $92 \%$ \\
\hline Negative predictive value & $80.769 \%$ & $83.5 \%$ & $78 \%$ \\
\hline
\end{tabular}

Table No. 22: Sensitivity specificity positive predictive value negative predictive value of mean arterial pressure with heart rate as gold standard at 5minutes for stress response to intubation

\begin{tabular}{|l|l|}
\hline & GA with ETT \\
\hline Sensitivity & $90.2 \%$ \\
\hline Specificity & $45 \%$ \\
\hline Positive Predictive Value & $81 \%$ \\
\hline Negative predictive value & $85 \%$ \\
\hline
\end{tabular}

\section{RESULTS}

Preinduction evaluation of hemodynamic parameters showed that there was no statistically significant difference between the four groups regarding heart rate, mean arterial pressure but there was a statistically significant difference between them regarding the perfusion index value $(\mathrm{p}=.0033, .0071,<.0001$ and .0081 in neuraxial, inhalational,tiva and general anesthesia with endotracheal intubation, respectively.

After 2. Minutes of induction there was significant increase of perfusion index. Perfusion index increase from $1.9162 \pm 0.6863$ to $2.27 \pm 0.6918$ with a p value of 0.0038 in neuraxial anesthesia. In inhalatonal anesthesia perfusion index increase from $2.06 \pm 0.6863$ to $2.66 \pm 0.6918$ with $p$ value of0.0266.In TIVA group perfusion index increase from $1.67 \pm 0.81$ to $2.48 \pm 1.02$ with $\mathrm{p}$ value of 0.0384 .In general anesthesia with ett perfusion index decreased from $1.77 \pm 0.814$ to $1.19 \pm 0.78$ with $p$ value of $<.0001$

In general anesthesia with endotracheal intubation there is a decrease of perfusion index from $1.77 \pm .8130$ to1.19 \pm .78 with $p$ value of, .0001. This decrease is mostly because of the stress response of intubation.

The heart rate changed from $82.396 \pm 10.58$ to $63.81 \pm 6.755$ in neuraxial anesthesia, from $81.7 \pm 6.484$ to66.81 \pm 6.755 in inhalational anesthesia; from $79.75 \pm 8.412$ to $65.35 \pm 8.731$ in tiva; from $78.75 \pm 7.474$ to $102.616 \pm 17.5$ in general anesthesia with ETT.

The mean arterial pressure changed from $82.39 \pm 10.58$ to $75.81 \pm 6.755$ in neuraxial anesthesia, from $86.9 \pm 7.6$ to71. $25 \pm 7.6$ in inhalational anesthesia; from $92.78 \pm 11.23$ to $65.35 \pm 8.7$ in TIVA; from $87.55 \pm 10.22$ to102.616 \pm 8.9 in general anesthesia with ETT. Since a rise in HR and pressor response is considered as a sympathetic pressor response to endotracheal intubation which is also considered as a transient "surgical stress" on the patients, and establishes that perfusion index is an added reliable parameter..

It establishes perfusion index as a reliable parameter, indicating a stress response of endotracheal intubation due to sympathetic stimulation.

Sensitivity of detecting onset of anaesthesia at 2 minutes was $66 \%, 66 \%$ and $58.055 \%$ in neuraxial ,inhalational, TIVA respectively and specificity was $26.6 \%, 37.03 \%$ and $17.24 \%$, in all three above mentioned group respectively using heart rate as gold standard where as sensitivity and specificity of 
MEAN ARTERIAL PRESSURE was 52\%, 20\% and 59\%, and 46\%, 40 and $30 \%$ \& in neuraxial anesthesia ,inhalatioal anesthesia and TIVA.

Sensitivity of perfusion index is very high and comparable to mean arterial pressure while detecting stress response of intubation with sensitivity of $92.4 \%$ and $92.5 \%$ respectively. Specificity of perfusion index was $28.5 \%$ where as specificity of mean arterial pressure was less indicating that perfusion is better and reliable parameter than mean arterial pressure.

These results prove that perfusion index is comparable and even more sensitive than other parameters to confirm the onset of anesthesia and to detect pressor response to intubation.

In general anesthesia with endotracheal intubation perfusion index decreased from 1.750+.78to1.250+.45 and heart rate and mean arterial pressure increased from $102.616+12.81$ to $109+14.81$ and 101.616 to $110.5+110.5+12.87$ respectively .Change perfusion index is significant .Pvalue <.024.

After 5 minutes of induction there was significant increase of perfusion index. Perfusion index increase from $1.9162 \pm .6863$ to $2.542 \pm .6918$ with a p value of.0028 in neuraxial anesthesia. In inhalatonal anesthesia,

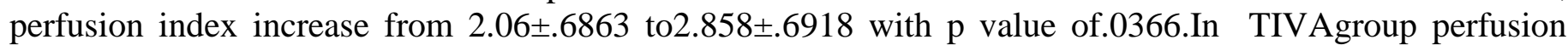
index increases from 1.67 \pm .81 to $2.79 \pm 1.021$ with $\mathrm{p}$ value of .0484 .In general anesthesia with ett perfusion index decreases from $1.77 \pm .814$ to $1.250 \pm .78$ with $p$ value of $<.0001$.

Sensitivity of perfusion index increases upto $100 \%, 93.4 \%$ and $90.56 \%$ in neuraxial anesthesia , TIVA andinhalational anesthesia respectively.

At 5 minutes sensitivity and specificity of mean arterial pressure was 90\%, 91\%,92\%, and 25\%, $28.57 \%$, and $20.8 \%$ respectively in neuraxial ,inhalational, and tiva.

Regarding pressor response to intubation at five minutes perfusion index has sensitivity of perfusion index detecting pressure responseto larygoscopy and intubation with sensitivity of $98.1 \%$ and specificity of $16.67 \%$ where as MAP value has sensitivity of $90.2 \%$ and specificity of $45 \%$ respectively.

\section{DISCUSSION}

PI is the numerical value of the amplitude of the plethysmo graphic pulse wave that is displayed on many pulse oximeters. The pulsating signal is indexed against the non-pulsating signal and expressed as ratio, it is commonly referred to as the "PI" $=\mathrm{AC} \times 100 / \mathrm{DC} \%$. In general terms, PI reflects the peripheral vasomotor tone. Low PI suggests peripheral vasoconstriction (or severe hypovolaemia) and high PI suggests vasodilation. PI is sensitive to several things such as temperature of the finger, exogenous vasoactive drugs, sympathetic nervous system tone (pain, anxiety, and so on) and stroke volume. The human cutaneous microcirculation has so far been studied by rather crude methods, such as plethysmography and $133 \mathrm{Xn}$ clearance. New sophisticated and noninvasive techniques are now available, with which the microcirculation of the skin can be continuously studied and measured for hours. With two such methods, i.e., Laser-Doppler flowmetry and dynamic capillaroscopy, fast dynamic fluctuations of the microcirculation can be followed. Under resting conditions, the total skin flow of a small area (1-2 mm2) varies consistently with time. These variations are caused by the arterial pulse, but also by active vasomotion with a frequency of about 4-8 cycles/min. These fluctuations can also be seen in single skin capillaries, but with a somewhat faster frequency: 6-10 cycles/min. The reaction of the cutaneous microcirculation to provocation tests, such as postocclusive reactive hyperemia response and venous occlusion $(50 \mathrm{~mm} \mathrm{Hg})$, can also be studied. The time to peak during postocclusive reactive hyperemia is very consistent. In response to venous congestion, capillary flow rate falls drastically, whereas Laser-Doppler flow is much less dramatically reduced. These discrepancies suggest that the Laser-Doppler flowmeter records blood flow in skin vessels in addition to the superficial, nutritional capillaries. The dynamic pattern of the skin microcirculation is altered in patients with hypertension, diabetes, and obstructive arterial disease.

Although there are several confounders influencing peripheral perfusion, determination of a decrease in PI $\leq 20 \%$ as a threshold for stress response seems to be reasonable.

Several studies investigated the effects of low peripheral perfusion caused by hypothermia, vasoconstriction or sympathetic nerve activity. Therefore, variation in PI is associated with potentially numerous causes, especially a small variation of $10 \%$ as observed in this study. The skin microcirculation is thus not under local control, but alsoreflects changes in sympathetic activity; the effect of these changes can be easily measures by spectral analysis In this context, Landsverk and colleagues ${ }^{34}$ investigated variations in 
plethysmographic waveform amplitude in deeply sedated and mechanically ventilated patients. They observed slow and large spontaneous oscillations in skin microcirculation per laser dopplerflowmetry related to the sympathetic nervous system and presumed that this mechanism determined the large variability of pulse oximetryphotoplethysmographic waveform signal.

The main finding in the present study was that percent PI response criterion achieved at five minutes $100 \%$ sensitivity in detecting the stress response to insertion of ET, On the other hand, MAPachieved sensitivity (90\%) in detecting haemodynamic stress responses in this population. This study is in concordance with study done by Atef $\mathrm{H}$ et al where P.I. criterion sensitivity was $100 \%$ and SBPand DBP wasrespectively $44.6 \%$ and $54.6 \%$ at 3 minutes of intubation ${ }^{61}$.

The efficacy of PI as an indicator for intravascular injection of epinephrine-containing epidural test dose in propofol-anaesthetisedadults was investigated by Hany A. Mowafiet al.(32) PI has been used to reflect an evident response with $100 \%$ sensitivity and specificity to epinephrine-containing epidural test dose. HR criterion was $95 \%$ reliable in detecting the intravascular injection of a simulated test dose containing epinephrine during TIVA. The sensitivity of SBP criterion was $90 \%$ in detection of IV epinephrine ${ }^{9,32}$

The efficacy of haemodynamic and T-wave criteria for detecting intravascular injection of epinephrine test dose in propofol-anaesthetised adults was investigated by Takahashi $\mathrm{S}$ et alThe minimal effective dose of epinephrine associated with $100 \%$ sensitivity and specificity was $10 \mu \mathrm{g}$ based on the SBP criterion, and was $5 \mu \mathrm{g}$ based on the HR and T-wave criteria.(32)

The sensitivity of PI andMAP criterions in the present study were 100\%, $90 \%$ respectively in the detection of stress postinsertion endotracheal intubation. This finding is similar to the previous studies that showed $100 \%$ sensitivity of PI.HR sensitivity criteria were (85\% up to $95 \%$ ) in mowafi and Takahashi studies ${ }^{9,32}$ However, these studies measured BPinvasively via arterial catheters, a practice that was not justified in our patients. The use of intermittent and non-invasive measurement of BP may have decreased its reliability to detect haemodynamic changes, because the temporary SBP increases may be easily missed between cycles. Failure to demonstrate high efficacy of the SBP and DBP criteria as markers for confirming stress response during propofol fentanyl isofluraneanaesthesia represents a limitation of these conventional haemodynamic responses.

Moreover, Galvin and colleagues ${ }^{19}$ found that P.I. increased with successful sympatholysis of peripheral and neuroaxialblock.P.I. can provide information about the relative balance between nociception and antinociception. It is quite likely that, in the next decade, the pulse oximeter will evolve into a multiparameter monitor with several PI-derived indices being displayed.

All of the studied patients were ASA I or II and the study didn't include any patients with hypovolaemia or hypertension, so results might not be applicable to patients with ASA grade more than II, hypovolaemia and hypertension etc. We didn't use invasive monitoring parameters as invasive blood pressure might prone the patient to risk withoutreasonable benefit.

\section{SUMMARY}

This study entitled "THE VARIABILITY OF PERFUSION INDEX AS A NEW PARAMETER IN DIFFERENT TYPES OF ANAESTHESIA TECHNIQUES AND ITS CORRELATION WITH SURGICAL STRESS AND RECOVERY FROM ANESTHESIA :AN OBSERVATIONAL CLINICAL STUDY"wascarried out in Departmentof Anesthesiology, Krishna Instituteof Medical Sciences, Karad duringa period of 2013-2015.

240 Patient were selected and randomized by computer .60 patient were selected for each group.30 male and 30 females patient were allocated to each group. Variation in perfusion index was studied separately in four technique of anesthesia namely neuraxial anesthesia, inhalational anesthesia, total intravenous anesthesia and general anesthesia with endotracheal intubation.

All patient were given 10 diazepam 12 hours before operation

All patients were adequately hydrated with $500 \mathrm{ml}$ of colloid 30 minutes before operation.

PI was monitored with using GE ohmedaTru Sat Tm model: GTM21089-1512-T3 .Upper and lower limits of P.I reported by the manufacturer is .02\%-20\%. The oximeter probe was attached to the middle finger of the patient's contra-lateral side of the B.P. monitoring. Finger was wrapped in a towel to minimise heat loss 
and contamination with ambience light. Simultaneous measurement of electrocardiographic H.R. and non invasiveoscillometric arterial blood pressure was measured. SPO2 was also measured.

Fluid administration was standardised to $10 \mathrm{ml} / \mathrm{kg} /$ minutes of ringer lactacte solution ambient temperature was maintained at 25- 26 degree centigrade.

Each patient was monitored 10 minutes before start of anesthesia and mean basal heart rate mean basal mean arterial pressure and mean basal perfusion index was calculated after observing the parameter for over five minutes.

Also average change in perfusion index, average change in heart rate and average change of mean arterial pressure was calculated by observing this parameter for 5 minutes by following formula. Maximum valueminimum value/2.

Perfusion index , heart rate and mean arterial pressure was noted at time of induction and then 2 minutes ,5 minutes 10 minutes and then every 5 minutes.

Significant criterion were set as following:

Change in Heart rate was taken significant if there was change in heart rate more than 10 beats per minutes.

Change in mean arterial pressure was taken significant if there was change in MAP was more than $10 \mathrm{~mm}$ of $\mathrm{Hg}$.

Change in perfusion index was taken significant when change in perfusion index was more than $20 \%$.

All three parameters were studied in neuraxial anesthesia, inhalatonalanesthesia, total intravenous anesthesia, and general anesthesia at the time of induction, 2 minutes ,5 minutes 10 minutes.

\section{STATISTICAL ANALYSIS}

Analysis was performed using the program ssps version 20 for windows.

The $95 \%$ confidence interval for sensitivity, specificity, and predictive values were calculated Wilson score method as described by Newcombe. Numerical data were presented asmean \pm standard categorical data as proportions (\%).

Statistical significance will be determined at 95\%level of confidence (difference will be considered statistically significant if $\mathrm{p}<0.5$ ).

One way ANOVA was used for comparision of mean differences in demographic data and baseline values of hemodynamic parameter and perfusion index between groups.For comparision of changes in the crieteria over time in different group , data was analysed by mixed design ANOVA.

Multiple tukey post hoc test for multiple comparision was then calculated for significant difference in ANOVA for analysis of categorical variables, the chi square test was used when any expected frequency was $<1$ or $20 \%$ of the expected frequency was $\leq 5$.

The measurement was taken by following methods:

Percentage change in perfusion index=P.I.max-P.I.min/P.I. $\min \times 100$

Preinduction average perfusion index index over 5 minutes $=\mathrm{PI} \max -\mathrm{PImin} / 2$

Preinduction heart rate variability in percentage over 5 minutes $=$ HR $\max -\mathrm{HR} \min / \mathrm{HR} \min \times 100$

Preinduction average hr over 5 minutes $=\mathrm{HR} \max -\mathrm{HR} \min / 2$

Preinduction MAP \% variability=

MAP max-MAPmin/MAP $\min \times 100$

Preinduction MAP average value over $5 \mathrm{~min}=$

MAP max -MAPmin/2

Percentage perfusion index variatioin overall operation=

PI max -PI min/ PImin $\times 10$

Sensitivity of detecting onset of anaesthesia at 2 minutes was $66 \%, 66 \%$ and $58.055 \%$ in neuraxial ,inhalational, TIVA respectively and specificity was $26.6 \%, 37.03 \%$ and $17.24 \%$, in all three above mentioned group respectively using heart rate as gold standard wehere as sensitivity and specificity of mean arterial pressure was 52\%, 20\% and 59\%, and 46\%, 40 and 30\% \& in neuraxial anesthesia ,inhalatioal anesthesia and TIVA.

Sensitivity of perfusion index is very high and comparable to mean arterial pressure while detecting stress response of intubation with sensitivity of $92.4 \%$ and $92.5 \%$ respectively. Specificity of perfusion index was 
$28.5 \%$ where as specificity of mean arterial pressure was less indicating that perfusion is better and reliable parameter than mean arterial pressure.

At 5 minutes sensitivity and specificity of mean arterial pressure was $90 \%, 91 \%, 92 \%$ and $25 \%, 28.57 \%$, and $20.8 \%$ respectively in neuraxial, inhalational, and TIVA.

Regarding pressor response to intubation at five minutes perfusion index has sensitivity of perfusion index detecting pressure response to larygoscopy and intubation with sensitivity of $98.1 \%$ and specificity of $16.67 \%$ where as MAP value has sensitivity of $90.2 \%$ and specificity of $45 \%$ respectively.

\section{CONCLUSION}

- Perfusion index is reliable and comparable to otherconventional hemodynamicparameters in an isovolumic conditions. Sensitivity of perfuson index is better or comparable in detecting the onset of anaesthesia compared to other haemodynamic parameters.

- Perfusion index is an early and reliable indicator of onset of neuraxialanaesthesia. Perfusion Index can predict the incidence of spinal anesthesia induced hypotension.

- Perfusion index is an early and sensitive indicator for onset of inhalational anesthesia and TIVA.

- Perfusion index is aearly and reliable indicator of onset of neuraxialanaesthesia. Perfusion Index can predict the incidence of spinal anesthesia induced hypotension.

- Monitoring P.I. during anesthetic management can be of tremendous help in respect to onset ofanaesthetic state, surgical pressure response and therefore surgical stress related to depth of anaesthesia,as well as recovery profile of anaesthesia.

- Monitoring P.I. is simple, non invasive and less expensive method of monitoring haemodynamic status along with conventional parameters, with an added advantage of being sensitive and specific in nature. Thoroug understanding of peripheral perfusion index using it in anaesthetic practice with all technical details and interpreting it in the light of haemodynamicmeleu

\section{BIBLIOGRAPHY}

1. Brian L. Partridge .Use of Pulse Oximetry as a Noninvasive Indicator of Intravascular Volume StatusJournal of Clinical Monitoring.1987; 3(4):263-268.

2. Evans ML, Geddes LA. An assessment of blood vessel vasoactivity using photoplethysmography.Med Instrum 1988; 22(5):29-32.

3. Hales JR, Stephens FR, Fawcett AA, Daniel K, Sheahan J, Westerman RA, James SB;Observations on a new non-invasive monitor of skin blood flow: ClinExpPharmacol Physiol. 1989 ;16(5):403-15

4. Bernardi L, Radaelli A, Solda PL, Coats AJ, Reeder M, Calciati A, Garrard CS, Sleight P.;Autonomic control of skin microvessels: assessment by power spectrum of photoplethysmographic waves.:ClinSci (Lond). 1996; 90(5):345-55.

5. ShamirM, EidelmanL.A., FlomanY, Kaplan L and PizovR;Pulse oximetryplethysmographic waveform during changes in blood volume:Br. J. Anaesth. (1999) 82 (2): 178-181.

6. Sinex, J.E. Pulse oximetry: principles and limitations. Am J Emerg Med. 1999; 17:59-67.

7. Kihara S, Brimacombe J, Yaguchi Y, Watanabe S, Taguchi N, Komatsuzaki T. Hemodynamic responses among three tracheal intubation devices in normotensive and hypertensive patients. AnesthAnalg 2003;96:890-5.

8. Shimoda O, Ikuta Y, Isayama S, Sakamoto M, Terasaki H. ;skin vasomotor reflex induced by laryngoscopy:compariso between Mc-coy and Macjntosh :British Journal of Anesthesia1997;84:120126

9. Takahashi S, Tanaka M, Toyooka H. ; The efficacy of hemodynamic and T-wave criteria for detecting intravascular injection of epinephrine test dose in propofol-anesthetized adults:anesthAnalg. $2002 ; 94(3): 717-22$;

10. Pole Y.Evolution of the pulse oximeter:International Congress Series the history of anaesthesia 2002; vol1242: 137-144. 
11. Robert F. Tamburro, John C. Ring, Kimberly Womback; Detection of PulsusParadoxus Associated With Large Pericardial Effusions in Pediatric Patients by Analysis of the Pulse-Oximetry Waveform: Pediatrics, 2002; vol 109 ( 4);1-8

12. Barak M, Ziser A, Greenberg A, Lischinsky S, Rosenberg B.; Hemodynamic and catecholamine response to tracheal intubation: direct laryngoscopy compared with fiberoptic intubation: J ClinAnesth. 2003; 15(2):132-6.

13. Koppel R, Druschel CM, Carter T, Goldberg BE, Mehta PN, Talwar R, Bierman FZ. ;Effectiveness of pulse oximetry screening for congenital heart disease in asymptomatic newborns: Pediatrics. 2003; 111(3):451-5.

14. Hagar H, Church S, Mandadi G, Pulley D, Kurz A. The perfusion index measured by a pulse oximeter indicates pain stimuli in anesthetized volunteers. Anesthesiology 2004; 101:A514.

15. Kakazu CZ, Chen BJ, Kwan WF. Masimo set technology using perfusion index is a sensitive indicator for epidural onset. Anesthesiology. 2005;103:A576.

16. Surgical stress response BrJA. 2003;109;B672.

17. Lima AP, Beelen P, Bakker J. Use of a peripheral perfusion index derived from the pulse oximetry signal as a noninvasive indicator of perfusion. Crit Care Med 2002; 30:1210-3.

18. Takenaka M, Greenberg A, Lischinsky S, Rosenberg B.; Hemodynamic and catecholamine response to tracheal intubation: direct laryngoscopy compared with fiberoptic intubation: J ClinAnesth. 2003; 15(2):132-6.

19. Galvin EM, Niehof S, Verbrugge SJ, Maissan I, Jahn A, Klein J, van Bommel J.Periipheral flow index is a reliable and early indicator of regional block success.:AnesthAnalg. 2006 ;103(1):239-43,

20. Feissel M, Teboul JL, Merlani P, Badie J, Faller JP, Bendjelid K Plethysmographic dynamic indices predict fluid responsiveness in septic ventilated patients: Intensive Care Med. 2007 ;33(6):993-9.

21. Awad AA, Haddadin AS, Tantawy H, Badr TM, Stout RG, Silverman DG, Shelley KH;The relationship between the photoplethysmographic waveform and systemic vascular resistance :J ClinMonitComput. 2007; 21(6):365-72.

22. Allen j.Photoplethysmography and its application in clinical physiological measurement; Physiol Meas. 2007;28(3):R1-39:

23. AGranelli Ad, Ostman-Smith I. ; Noninvasive peripheral perfusion index as a possible tool for screening for critical left heart obstruction. ActaPaediatr. 2007 ;96(10):1455-9.

24. Severinghaus JW. Takuo Aoyagi: discovery of pulseoximetry :anesthAnalg. 2007; 105(6 Suppl):S16.

25. Shelley KH .Photoplethysmography: Beyond the Calculation of Arterial Oxygen Saturation and Heart Rate : AnesthAnalg 2007;105(6 suppl):S1-4

26. Gesquiere, M.J., Awad, A.A., Silverman, D.G. et al, Impact of withdrawal of $450 \mathrm{ml}$ of blood on respiration-induced oscillations of the ear plethysmographic waveform. J ClinMonitComput. 2007;21(5):277-282.

27. Raamat R, Jagomägi K, Talts J. Calibrated ;photoplethysmographic estimation of digital pulse volume and arterial compliance: ClinPhysiolFunct Imaging. 2007 ;27(6):354-62.

28. Frey B, Waldvogel K, BalmerC;Clinical applications of photoplethysmography in paediatric intensive care.:intensive Care Med. 2008 ;34(3):578-82.

29. Mowafi HA, Arab SA, Ismail SA, Al-Ghamdi AA, Al-Metwalli RR ;Plethysmographic pulse wave amplitude is an effective indicator for intravascular injection of epinephrine-containing epidural test dose in sevoflurane-anesthetized pediatric patients.:AnesthAnalg. 2008;107(5):1536-41.

30. Landsverk SA, Hoiseth LO, Kvandal P, Hisdal J, Skare O, Kirkeboen KA. Poor agreement between respiratory variations in pulse oximetryphotoplethysmographic wave form amplitude and pulse pressure in intensive care unit patients.anesthesiology 2008;81:148-54

31. M. Koch, D. De Backer, J. L. Vincent, L. Barvais, D. Hennart and D. Schmartz1;Effects of propofol on human microcirculation:Br. J. Anaesth. 2008101 (4): 473-478.

32. Li SQ, Luo BR, Wang BG; Correlation of tip perfusion index with hemodynamics and catecholamines in patients undergoing general anesthesia: Zhonghuayixuezazhi : 2008, 88(17):11771180 
33. Reisner A, ShaltisPA, McCmbieD, Asada HH. Utility of the photoplethysmography in circulatory monitoring.Anesthesiology 2008;108:950-8

34. KorhonenA. and Yli-hankala.A. Photoplethysmography and nociception; Acta Anaesthesiologica Scandinavica: 2009:53( 8): 975-985,.

35. MustolaS, ParkkariT, HuikuM, KymäläinenM and ToivonenJ;Performance of Surgical Stress Index during Sevoflurane-Fentanyl and Isoflurane- Fentanyl Anesthesia: Anesthesiology Research and Practice 2010;1242; $1-5$

36. CresiF, PelleE, CalabreseR, CostaL, FarinassoD and SilvestroL;Perfusion index variations in clinically and hemodynamically stable preterm newborns in the first week of life Italian Journal of Pediatrics 2010, 36(6) .43-49

37. Sandroni C etal; Accuracy of plethysmographic indices as predictors of fluid responsiveness in mechanically ventilated adults: A systematic review and meta-analysis:Intensive Care Medicine 2012; 38(9):1429-37.

38. Sahni R, Schulze KF, Ohira-Kist K, Kashyap S, Myers MM, Fifer WP.interactions among peripheral perfusion, cardiac activity, oxygen saturation, thermal profile and body position in growing low birth weight infants:ctaPaediatr. 2010;99(1):135-9.

39. Xinzhong C. KoheiGodai · Maiko Hasegawa-Moriyama - Tamotsu Kuniyoshi · Akira Matsunaga · Yuichi Kanmura . TomoyukiKuwaki;Perfusion index as a possible predictor for postanestheticshivering:Journal of Anesthesia2013; 28(1). 123-27

40. Donald H Arnold,,Cathy A Jenkins, and Tina V Hartert,Noninvasive assessment of asthma severity using pulse oximeterplethysmograph estimate of pulsusparadoxusphysiology:MCPulm Med. 2010; 10: 17.

41. Hood, Julian A.; Wilson, R. Jonathan T. ;Pleth Variability Index to Predict Fluid Responsiveness in Colorectal Surgery:Anesthesia\&Analgesia:November 2011 - 113 ( 5) 1058-1063

42. Abdullah $\mathrm{M}$ et al;Goal directed fluid optimization using pleth variability index versus corrected flow time in cirrotic patients undergoing major abdominal surgery;Egyptian journal ;2011;28;23-28.

43. Bonhomme V, Uutela K, Hans G, Maquoi I, Born JD, Brichant JF, Lam;Comparison of the surgical Pleth Index ${ }^{\mathrm{TM}}$ with haemodynamic variables to assess nociception-anti-nociception balance during general anaesthesia.:Br J Anaesth. 2011;106(1):101-11.

44. DouglasE,Perfusion Index Used as Tool To Confirm Epidural Placement: Clinical Anesthesiology 2006 ; vol 32:06-45

45. Cannesson M, Desebbe O, Rosamel P, Delannoy B, Robin J, Bastien O, Lehot JJ.Pleth variability index to monitor the respiratory variations in the pulse oximeter plethysmographic waveform amplitude and predict fluid responsiveness in the operating theatre; Br J Anaesth. 2008;101(2):2006.

46. Takeyamam,MatsunagaA,KakihanaY,MasudaM,KuniyoshiT,KanmuraY.Impact of skin incision on thePVI.J ClinComput2011;4(25):215-2

47. Mohamed Elgendi;On the Analysis of Fingertip Photoplethysmogram Signals: CurrCardiol Rev. 2012; 8(1): 14-25

48. Hajime Yamazaki, Junichi Nishiyama, and Toshiyasu Suzuki;Use of perfusion index from pulse oximetry to determine efficacy of stellate ganglion block:LocalRegAnesth. 2012; 5: 9-14.

49. J. Y. Yin andK. M. Ho2.Use of plethysmographic variability index derived from the Massimo® pulse oximeter to predict fluid or preload responsiveness: a systematic review and metaanalysisAnaesthesia: $2012 ; 67(7)$ : 777-783,

50. Hamunen K, Kontinen V, Hakala E, Talke P, Paloheimo M, Kalso E. Effect of pain on autonomic nervous system indices derived from photoplethysmography in healthy volunteers: $\mathrm{Br} \mathrm{J}$ Anaesth. 2012;108(5):838-44.

51. Sahni R, Schulze KF, Ohira-Kist K, Kashyap S, Myers MM, Fifer WP.interactions among peripheral perfusion, cardiac activity, oxygen saturation, thermal profile and body position in growing low birth weight infants:ctaPaediatr. $2010 ; 99(1): 135-9$.

52. Jung-Nam Kwon and Whan-Bong Lee ;Utility of digital pulse oximetry in the screening of lower extremity arterial disease: J Korean Surg Soc. 2012 ; 82(2): 94-100. 
53. Toyama S, Kakumoto M, Morioka M, Matsuoka K, Omatsu H, Tagaito $\mathrm{Y}$, Numai $\mathrm{T}$, ShimoyamaM.;Perfusion index derived from a pulse oximeter can predict the incidence of hypotension during spinal anaesthesia for Caesarean delivery:. Br J Anaesth. 2013;111(2):235-41.

54. .Kinoshita M, Hawkes CP, Ryan CA, Dempsey EM. ;Perfusion index in the very preterm infant:ActaPaediatr. $2013 ; 102(9): 398-401$.

55. Xu Z, Zhang J, Xia Y, Deng X. Accuracy of pulse oximeter perfusion index in thoracic epidural anesthesia under basal general anesthesia:Int J ClinExp Med. 2014 ;7(7):1728-34.

56. Zifengxu, Bartunek A, Gilly H.;Effect of local limb temperature on pulse oximetry and the plethysmographic pulse wave: Int J ClinMonitComput. 1997 ;14(1):17-22.

57. Kowalczyk M, FijałkowskaA, NestorowiczA ;New-generation pulse oximetry for the assessment of peripheral perfusion during general anaesthesia - a comparison between propofol and desflurane :AnaesthesiologyIntensive Therapy 2013, 45( 3) 134-144

58. van Genderen ME, Bartels SA, Lima A, Bezemer R, Ince C, Bakker J, van BommelJ.;Peripheral perfusion index as an early predictor for central hypovolemia in awake healthy volunteers.:AnesthAnalg. 2013;116(2):351-6.

59. Hosam M Atef, Salah Abd Fattah, Mohammed EmadAbdGaffer;Perfusion index versus non-invasive hemodynamic parameters during insertion of i-gel, classic laryngeal mask airway and endotracheal tube:ndian J Anaesth. 2013; 57(2): 156-162.

60. Chiharu Kuroki · KoheiGodai · Maiko Hasegawa-Moriyama · Tamotsu Kuniyoshi · Akira Matsunaga - Yuichi Kanmura - TomoyukiKuwaki;Perfusion index as a possible predictor for postanestheticshivering:Journal of Anesthesia ;2013; 28(1).176-186

61. NishimuraT, NakaeA, ShibataM, MashimoT,and FujinoY;Age-related and sex-related changes in perfusion index in response to noxious electrical stimulation in healthy subjects: J Pain Res. 2014; 7 : 91-97.

62. Loupec T1, Nanadoumgar H, Frasca D, Petitpas F, Laksiri L, Baudouin D, Debaene B, DahyotFizelier C, Mimoz O: Pleth variability index predicts fluid responsiveness in critically ill patients:Crit Care Med. 2011;39(2):294-9.

63. Hakan N, Dilli D, Zenciroglu A, Aydin M, OkumusN.;Reference values of perfusion indices in hemodynamically stable newborns during the early neonatal period:Eur J Pediatr. 2014 ;173(5):597602.

64. IXu Z, Zhang J, Xia Y, Deng X. Accuracy of pulse oximeter perfusion index in thoracic epidural anesthesia under basal general anesthesia:Int J ClinExp Med. 2014 ;7(7):1728-34.

65. van Genderen ME, Bartels SA, Lima A, Bezemer R, Ince C, Bakker J, van BommelJ.;Peripheral perfusion index as an early predictor for central hypovolemia in awake healthy volunteers.:AnesthAnalg. 2013 ;116(2):351-6.

66. Jardim J, Rocha R, Silva G, Guimarães H. Peripheral perfusion index- reference range in healthy Portuguese term newborns. J PediatrNeonat Individual Med. 2014;3(1):312-317

67. Huai-wu He,Da-wei Liu Yun Long, and Xiao-ting Wang:The peripheral perfusion index and transcutaneous oxygen challenge test are predictive of mortality in septic patients after resuscitation rit Care. 2013; 17(3): R116.critical Care. 2013; 17(3): R116.

68. Mousa WF. ;Effect of hypercapnia on pleth variability index during stable propofol: Remifentanilanesthesia:Saudi J Anaesth. 2013 Jul;7(3):234-7.

69. SawadaaY, Tanaka G, Ken-ichi,Yamakosh Y; Normalized pulse volume (NPV) derived photoplethysmographically as a more valid measure of the finger vascular tone :International Journal of Psychophysiology2001;41(1);1-10.

70. Fagrell B.Dynamics of skin microcirculation in humans: J CardiovascPharmacol. 1985;7 (Suppl 3):53-8. 
ANNEXURE-I

PERFORMA

THE VARIABILITY OF PERFUSION INDEX AS A NEW PARAMETER IN DIFFERENT TYPES OF ANAESTHESIA TECHNIQUES AND ITS CORRELATION WITH SURGICAL STRESS AND RECOVERY FROM ANESTHESIA: AN OBSERVATIONAL CLINICAL STUDY

Name of patient:

Hospital no:

ASA status:

Age:

Weight:

Duration of operation:

Preoperative evaluation:

Significant abnormality in investigation

Preinduction percentage perfusion index variability over 5 mkinutes=

P.I.max-P.I.min/P.I. $\min _{\times 100}$

Preinduction average perfusion index over 5 minutes $=$

PI max- PImin/2

Preinduction heart rate variability in percentage over 5 minutes $=$

HR $\max -$ HR $\min /$ HR $\min \times 100$

Preinduction average hr over 5 minutes $=$

HR max -HR min $/ 2$

Preinduction MAP \% variability=

MAP max-MAPmin/MAP $\min \times 100$

Preinduction MAP average value over $5 \mathrm{~min}=$

MAP max -MAPmin/2

Percentage perfusion index variatioin overall operation $=$

PI max -PI min/ PImin min $\times 100$

Preoperative PI value:

Preoperative HRvalue:

Preoperative MAP value:

\begin{tabular}{|l|l|l|l|}
\hline $\begin{array}{l}\text { Duration of operation P.I. heart rate } \\
\text { M.A.P. from time of Induction }\end{array}$ & PERFSION INDEX & HEART RATE & $\begin{array}{l}\text { MEAN ARTERIAL } \\
\text { PRESSURE }\end{array}$ \\
\hline 0 & & & \\
\hline 2 & & & \\
\hline 5 & & & \\
\hline 10 & & & \\
\hline 15 & & & \\
\hline 18 & & & \\
\hline 21 & & & \\
\hline 24 & & & \\
\hline 27 & & & \\
\hline 30 & & & \\
\hline 33 & & & \\
\hline 36 & & & \\
\hline 39 & & & \\
\hline 42 & & & \\
\hline 45 & & & \\
\hline 48 & & & \\
\hline 51 & & & \\
\hline 54 & & & \\
\hline
\end{tabular}




\begin{tabular}{lrl}
\multicolumn{2}{c}{ KEY TO MASTER CHART } \\
M.A.P & - & Mean Arterial Pressure \\
P.I. & - & Perfusion Index \\
H.R. & - & Heart Rate \\
MIN & - & Minutes \\
ABS & - & Absolute \\
BSL & - & Baseline \\
$\%$ & - & Percentage \\
C/S & - & Cesarean Section \\
I/D & - & Incision Drainge \\
D\&C & - & Dilatation And Currettage \\
M & - & Male \\
F & - & Female \\
Sr NO. & - & Serial Number
\end{tabular}

